\title{
HEDGING UNDER GENERALIZED GOOD-DEAL BOUNDS AND MODEL UNCERTAINTY
}

\author{
DIRK BECHERER AND KLEBERT KENTIA
}

\begin{abstract}
We study a notion of good-deal hedging that corresponds to good-deal valuation and is described by a uniform supermartingale property for the tracking errors of hedging strategies. For generalized good-deal constraints, defined in terms of correspondences for the Girsanov kernels of pricing measures, constructive results on good-deal hedges and valuations are derived from backward stochastic differential equations, including new examples with explicit formulas. Under model uncertainty about the market prices of risk of hedging assets, a robust approach leads to a reduction or even elimination of a speculative component in good-deal hedging, which is shown to be equivalent to a global risk-minimization the sense of Föllmer and Sondermann (1986) if uncertainty is sufficiently large.
\end{abstract}

\section{INTRODUCTION}

The theory of good-deal bounds offers a valuation approach for contingent claims in incomplete markets, where the issuer of derivative contracts cannot eliminate his risk entirely by dynamic hedging but only partially. Good deal bounds are defined as economically meaningful valuation bounds by ruling out not only arbitrage opportunities but also a suitable notion of deals that are 'too good'. The most cited work appears to be Cochrane and Requejo (2000), and for pioneering conceptual contributions we refer also to Cerný and Hodges (2002); Cerný (2003); Jaschke and Küchler (2001). Mathematical theory in continuous time, where earlier contributions have partly relied on heuristic arguments, has been made rigorous and generalized in Björk and Slinko (2006); Klöppel and Schweizer (2007). Inherent to the concept of good-deal valuation bounds is already a certain notion of robustness, in that it considers a 'no good-deal' range of arbitrage-free valuations over a suitable set of risk neutral good-deal pricing measures, instead of using one single calibrated pricing measure. Such is conservative for risks that cannot be perfectly hedged, e.g. illiquid exotic options, and also with respect to mark-to-market losses that could arise during the regular recalibration of risk-neutral valuation models to the actual market prices of liquid options, if model predictions for price relations differ from reality. Good deals have been defined mostly in terms of Sharpe ratios (Cochrane and Requejo, 2000; Cerný and Hodges, 2002; Cerný, 2003; Björk and Slinko, 2006; Bayraktar and Young, 2008; Delong, 2012; Carassus and Temam, 2014) or alternatively by some notion of (quadratic or else) expected utilities (cf. Cerný, 2003; Klöppel and Schweizer, 2007; Becherer, 2009). Either definition relates to a single objective probability $P$ in terms of market prices of risk. Hence robustness and model uncertainty, being important for finance and decision theory (cf. Cont, 2006; Hansen and Sargent, 2001; Gilboa and Schmeidler, 1989) in general, are relevant problems for good-deal theory. This theory has evolved for some time purely as a pricing theory; in the conclusions of Björk and Slinko (2006) a quest for a corresponding dual theory of good deal hedging was noted as a "challenging open problem". Different notions for good deal hedging have been proposed in Becherer (2009), where good-deal hedging strategies are defined as minimizers of a-priori risk measure in spirit of Barrieu and El Karoui (2009), or Carassus and Temam (2014) who suggest minimum variance hedging and demonstrate numerically that both approaches perform comparably well.

The present paper is concerned with approaches to good deal hedging (as in Becherer, 2009) under Knightian model uncertainty (ambiguity) about the objective probability, with respect to which good deals are defined. To this end, we pose the good-deal valuation and hedging problem in a framework

2010 Mathematics Subject Classification. 60G44, 91G10, 93E20, 91B06.

Key words and phrases. Good-deal bounds, good-deal hedging, model uncertainty, incomplete markets, multiple priors, backward stochastic differential equations.

Support from the German Science Foundation DFG via the Berlin Mathematical School and the Research Training Group 1845 Sto-A is gratefully acknowledged. 
with multiple (uncertain) priors, and follow a robust worst-case approach as in Gilboa and Schmeidler (1989). Good deal valuation fits into the theory of dynamic monetary convex, even coherent, risk measures (or monetary utility functionals), for which a rich theory in high generality exists (see e.g. Klöppel and Schweizer, 2007; Bion-Nadal and Di Nunno, 2013; Drapeau and Kupper, 2013). On dual representations and time consistency of value functions there seems little to add. Instead, we contribute constructive and qualitative results on the (robust, good-deal) hedging strategies. Hedging strategies arise as minimizers for suitable a-priori coherent risk measures under optimal risk sharing with the market. Literature on robust utility maximizing strategies appears richer than on robust hedging strategies (except on superhedging (cf. El Karoui and Quenez, 1995), which excludes losses but is often too expensive); for innovative approaches and many more references on the former we refer to Dow and Werlang (1992); Quenez (2004); Garlappi et al. (2007); Schied (2008); Seifried (2010); Biagini and Pinar (2016). Results on robust good deal hedging in the interesting recent work by Boyarchenko et al. (2014) are different from ours. They study a different uncertainty-penalized preference functional and obtain numerical results in discrete time, whereas we use dynamic coherent risk measures in continuous time and focus mostly on analytical results.

To derive the optimality equations, we systematically use backward stochastic differential equations (BSDEs) (see El Karoui et al., 1997), which in some informal way already appear present in (Cochrane and Requejo, 2000, Sect.II.C.6), instead of Markovian dynamical programming methods and PDE (as Cochrane and Requejo, 2000; Björk and Slinko, 2006). To this end, Section 2 formulates an abstract framework of good-deal constraints, described by predictable correspondences for the Girsanov kernels of the respective pricing measures, that is sufficiently general for all later sections. It incorporates the common radial good-deal constraints on Girsanov kernels, defined by (constant) scalar bounds on Euclidian norms, that are predominant in the good deal literature (cf. Björk and Slinko, 2006; Bayraktar and Young, 2008; Bondarenko and Longarela, 2009; Donnelly, 2011; Marroquín-Martínez and Moreno, 2013) but also extensions like ellipsoidal constraints, that still permit explicit analytic generators in the BSDEs of interest, being efficient for Monte Carlo approximation. Notably, the generalized constraints are needed to cover relevant examples in Section 4 for model uncertainty about the market prices of risk of the assets that are available in the (incomplete) market for partial hedging, cf. Remarks 4.7-4.14. Section 3 studies good-deal hedging strategies and provides new case studies with closed-form solutions. For an exchange option between tradeable and non-tradeable assets, the good-deal bounds are given by a Margrabe formula with adjusted input parameters. For the stochastic volatility model by Heston, we obtain semi-explicit formulas under good-deal constraints for pricing measures, which restrict the mean reversion level of the stochastic variance process to some interval. This shows, how a conservative valuation for volatility related risks could be obtained in a (simpler) dominated setup without singularity of measures. To illustrate to which extend our BSDE solutions can be computed by efficient but generic Monte Carlo methods, complementing numerical results by Carassus and Temam (2014), we investigate errors between an efficient but generic Monte Carlo approximation and our analytic formula in a 4-dimensional example. In a framework without uncertainty, good deal hedging strategies are shown to comprise a speculative component to compensate for unhedgeable risks, see Section 3.1.

Good deal hedges and valuations that are robust with respect to uncertainty are derived in the main Section 4. This builds on the analysis of previous sections since the problem with uncertain multiple priors can be related to a respective problem without uncertainty for suitably enlarged correspondences, within a (dominated) setup of absolutely continuous probability measures. A worst-case approach naturally leads to robust valuation by the widest good-deal bounds that are obtained over all (dominated) probabilistic models under consideration. We show that there is also a corresponding notion for robust dynamic hedging. Indeed, there exists a hedging strategy such that its tracking error, given by the dynamic variation of the robust good-deal bounds plus the profits and losses from hedging, exhibits a supermartingale property uniformly over all a-priori valuation measures with respect to all priors. This means, that hedging strategies are at least mean-self-financing (in the sense of Schweizer, 2001) in a uniform sense. By saddle point arguments we derive a minmax identity, that shows how the robust gooddeal hedging strategy is given by the (ordinary) good-deal hedging strategy with respect to a suitable worst case measure (for a related result in the context of robust utility maximization cf. e.g. Schied (2008)). Both are identified constructively, see Remark 4.14. As to be expected, a robust approach to uncertainty reduces the speculative component of the good-deal hedging strategy. As a further contribution, we prove that if the uncertainty is large enough in relation to the good-deal constraints, 
then the robust good-deal hedging strategy does no longer include any speculative component, but coincides with a (globally) risk minimizing strategy in spirit of Föllmer and Sondermann (1986) (cf. the survey of Schweizer, 2001) with respect to a suitable measure. This offers theoretical support to the commonly held perception that hedging should abstain from speculative objectives and offers a new justification for risk-minimization, which may be criticized at first sight for using a quadratic hedging criterion that penalizes gains and losses alike.

\section{MAThematical FRAMEWORK AND PRELIMINARIES}

We work on a filtered probability space $(\Omega, \mathcal{F}, \mathbb{F}, P)$ with time horizon $T<\infty$; the filtration $\mathbb{F}=\left(\mathcal{F}_{t}\right)_{t \leq T}$ generated by an $n$-dimensional Brownian motion $W$, augmented with $P$-null-sets, satisfying the usual conditions. Let $\mathcal{F}=\mathcal{F}_{T}$. Inequalities between random variables (processes) are meant to hold almost everywhere with respect to $P$ (resp. $P \otimes d t$ ). For stopping times $\tau \leq T$, the conditional expectation given $\mathcal{F}_{\tau}$ under a probability measure $Q$ is denoted by $E_{\tau}^{Q}[\cdot]$. We write $E_{\tau}=E_{\tau}^{P}$ if there is no ambiguity about $P$. $L^{p}\left(\mathbb{R}^{m}, Q\right), p \in[1, \infty)$, (or $L^{\infty}\left(\mathbb{R}^{m}, Q\right)$ ) denotes the space of $\mathcal{F}_{T}$-measurable $\mathbb{R}^{m}$-valued random variables $X$ with $\|X\|_{L^{p}(Q)}^{p}=E^{Q}\left[|X|^{p}\right]<\infty$ (resp. $X Q$-essentially bounded). $\mathcal{P}$ denotes the predictable $\sigma$-field on $[0, T] \times \Omega$. Stochastic integrals of predictable integrands $H$ with respect to semimartingales $S$ are denoted $H \cdot S=\int_{0}^{*} H_{t}^{\mathrm{tr}} d S_{t}$. Let $\mathcal{H}^{p}\left(\mathbb{R}^{m}, Q\right)$ denote the space of predictable $\mathbb{R}^{m}$-valued processes $Z$ with $\|Z\|_{\mathcal{H}^{p}(Q)}^{p}=E^{Q}\left[\left(\int_{0}^{T}\left|Z_{s}\right|^{2} d s\right)^{\frac{p}{2}}\right]<\infty$, and $\mathcal{S}^{p}(Q)$ that of càdlàg semimartingales $Y$ with $\|Y\|_{\mathcal{S}^{p}(Q)}=\left\|\sup _{t \leq T}\left|Y_{t}\right|\right\|_{L^{p}(Q)}<\infty$ If the dimension is clear, we just write $L^{p}(Q)$ and $\mathcal{H}^{p}(Q)$, and if $Q=P$ just $L^{p}, \mathcal{H}^{p}$ and $\mathcal{S}^{p}$, for $p \in[1, \infty]$. The Euclidean norm of a matrix $M \in \mathbb{R}^{n \times d}$ is $|M|:=\left(\operatorname{Tr} M M^{\text {tr }}\right)^{1 / 2}$ and its usual operator norm is denoted by $\|M\|$.

We will make use of classical theory of BSDEs (Pardoux and Peng, 1990; El Karoui et al., 1997). BSDEs are stochastic differential equations of the type

$$
-d Y_{t}=f\left(t, Y_{t}, Z_{t}\right) d t-Z_{t}^{\mathrm{tr}} d W_{t}, \text { for } t \leq T, \text { and } Y_{T}=X
$$

where the terminal condition $X$ is an $\mathcal{F}_{T}$-measurable random variable and the generator $f: \Omega \times[0, T] \times$ $\mathbb{R}^{1+n} \rightarrow \mathbb{R}$ a $\mathcal{P} \otimes \mathcal{B}\left(\mathbb{R}^{1+n}\right)$ - $\mathcal{B}(\mathbb{R})$-measurable function. They are well established in mathematical economics. A pair $(f, X)$ constitutes standard parameters (also called data) for a BSDE (2.1) if $X \in L^{2}, f(\cdot, 0,0)$ is in $\mathcal{H}^{2}$ and $f$ is uniformly Lipschitz in $y$ and $z$, i.e. there exists $L \in(0, \infty)$ such that $P \otimes d t$-a.e. $\left|f(\omega, t, y, z)-f\left(\omega, t, y^{\prime}, z^{\prime}\right)\right| \leq L\left(\left|y-y^{\prime}\right|+\left|z-z^{\prime}\right|\right)$ for all $y, z, y^{\prime}, z^{\prime}$. A solution of the $\operatorname{BSDE}(2.1)$ is a couple $(Y, Z)$ of processes such that $Y$ is real-valued continuous, adapted, and $Z$ is $\mathbb{R}^{n}$-valued predictable and satisfies $\int_{0}^{T}\left|Z_{t}\right|^{2} d t<\infty$. For standard parameters $(f, X)$ there exists a unique solution $(Y, Z) \in \mathcal{S}^{2} \times \mathcal{H}^{2}$ to the BSDE (2.1), (El Karoui et al., 1997, Thm.2.1). Let us refer to BSDEs with standard parameters as classical and to the solution to such BSDEs as standard. A comparison theorem (El Karoui et al., 1997, Prop.3.1) is very useful for optimal control problems stated in terms of classical BSDEs: Given standard BSDE solutions $(Y, Z),\left(Y^{a}, Z^{a}\right)_{a \in A}$ for a family of standard parameters $(f, X),\left(f^{a}, X^{a}\right)_{a \in A}$, if there exists $\bar{a} \in A$ such that $f\left(t, Y_{t}, Z_{t}\right)=\underset{a \in A}{\operatorname{essinf}} f^{a}\left(t, Y_{t}, Z_{t}\right)=f^{\bar{a}}\left(t, Y_{t}, Z_{t}\right), P \otimes d t$-a.e., and $X=\underset{a \in A}{\operatorname{ess} \inf } X^{a}=X^{\bar{a}}$, then $Y_{t}=\underset{a \in A}{\operatorname{essinf}} Y_{t}^{a}=Y_{t}^{\bar{a}}$ holds for all $t \leq T$.

Section 2.1 will specify a financial market with $d$ risky assets whose discounted price processes $S^{i}$ $(i \leq d)$ with respect to a fixed numéraire asset (with unit price $S^{0}=1$ ) are non-negative locally bounded semimartingales. The set of equivalent local martingale measures (risk neutral pricing measures) is denoted by $\mathcal{M}^{e}:=\mathcal{M}^{e}(S)$ and we assume $\mathcal{M}^{e} \neq \emptyset$, i.e. there is no free lunch with vanishing risk in the sense of Delbaen and Schachermayer (1994). The market is incomplete with $\mathcal{M}^{e}$ being of infinite cardinality if $d<n$. Following Rockafellar (1976), we define generalized good-deal bounds by using abstract predictable correspondences (multifunctions) $C$ defined on $[0, T] \times \Omega$ with non-empty compact and convex values $C_{t}(\omega) \subset \mathbb{R}^{n}$, with predictability meaning that for each closed set $F \subset \mathbb{R}^{n}$, the set $C^{-1}(F):=\left\{(t, \omega) \in[0, T] \times \Omega: C_{t}(\omega) \cap F \neq \emptyset\right\}$ is predictable. More specific examples, e.g. for ellipsoidal constraints, will exhibit (semi)explicit solutions for optimizers. We write $C:[0, T] \times \Omega \rightsquigarrow \mathbb{R}^{n}$ with " $\rightsquigarrow$ " for a set-valued mapping $C$, and $\lambda \in C$ means that the predictable function $\lambda$ is a selection of $C$, i.e. $\lambda_{t}(\omega) \in C_{t}(\omega)$ holds on $[0, T] \times \Omega$. Note that the seemingly weaker existence of a $\tilde{\lambda}$ with $\tilde{\lambda}_{t}(\omega) \in C_{t}(\omega)$ just $P \otimes d t$-a.e. on $[0, T] \times \Omega$ implies existence of a selection $\lambda \in C$, if $0 \in C \neq \emptyset$ (as often later). Throughout, a standard correspondence will refer to a predictable one, whose values are 
non-empty, compact and convex. Let $C:[0, T] \times \Omega \rightsquigarrow \mathbb{R}^{n}$ be a fixed standard correspondence with $0 \in C$. The set $\mathcal{Q}^{\text {ngd }}:=\mathcal{Q}^{\text {ngd }}(S)$ of (equivalent) no-good-deal measures is given by

$$
\mathcal{Q}^{\text {ngd }}(S):=\left\{Q \in \mathcal{M}^{e} \mid d Q / d P=\mathcal{E}(\lambda \cdot W), \lambda \text { predictable, bounded, } \lambda \in C\right\} .
$$

For good-deal valuation and hedging results later, concrete assumptions (e.g. Assumption 2.3) may ensure that all selections $\lambda$ of $C$ are bounded automatically. We remark that for good time-consistency properties, good-deal constraints should be specified locally in time (Klöppel and Schweizer, 2007). For contingent claims $X$ (bounded or sufficiently integrable), upper and lower good-deal valuation bounds

$$
\pi_{t}^{l}(X):=\underset{Q \in \mathcal{Q}^{\text {ngd }}}{\operatorname{ess} \inf _{t}} E^{Q}[X] \quad \text { and } \quad \pi_{t}^{u}(X):=\underset{Q \in \mathcal{Q}^{\text {ngd }}}{\operatorname{ess} \sup _{t}} E_{t}^{Q}[X], \quad t \in[0, T]
$$

are defined over a suitable (yet abstract) set of no good deal pricing measures $\mathcal{Q}^{\text {ngd }}$. Hence $\pi_{t}^{u}(X)$ (respectively $\left.\pi_{t}^{l}(X)\right)$ can be seen as the highest (lowest) valuation that does not permit too good deals to the seller (buyer). Since $\pi^{l}(X)=-\pi^{u}(-X)$, further analysis can be restricted to $\pi^{u}(X)$.

Referring to El Karoui and Quenez (1995), we note that the good deal bounds in (2.3) are within the interval of no-arbitrage prices, whose upper (lower) bound corresponds to the minimal superreplication cost for the seller (respectively buyer) of the claim $X$; this follows from $(2.3)$ and $\mathcal{Q}^{\text {ngd }} \subset \mathcal{M}^{e}$. In the same context, one may note that the BSDE (2.8), which will describe the good-deal valuation bound $\pi^{u}(X)$, involves a non-negative term that can be interpreted as a penalization which is proportional to the length of the 'non-hedgeable' part $\Pi_{t}^{\perp}\left(Z_{t}\right)$ of $Z$ (cf. (2.5)); In the common (radial, cf. Section 3.1) specification of good deals in the literature, this term reads as $k\left|\Pi_{t}^{\perp}\left(Z_{t}\right)\right|$ for some scalar parameter $k>0$, so it is intuitive that the good deal bound tends to the superreplication price for $k \rightarrow \infty$.

As mentioned already in the introduction, the definition (2.3) in itself could already be viewed as a robust representation in a sense (over $Q$ 's). For the purposes of the present paper however, the correspondence $C$ and the respective set $\mathcal{Q}^{\text {ngd }}$ of no good deal measures are (at first) given with respect to one objective real world measure $P$ (cf. remarks after (2.4)). To be clear in our use of terminology, we will in the sequel restrict our use of terms model uncertainty, ambiguity or robust hedging/valuation to situations with Knightian model uncertainty about $P$. Note that the use of terminology in some literature (e.g. Delong, 2012) is different, where those terms may instead refer to representations like (2.3). Definition (2.2) implies that density processes of measures $Q \in \mathcal{Q}^{\text {ngd }}$ are in $\mathcal{S}^{p}, p \in[1, \infty)$. Hence $X \in L^{2}=L^{2}(P) \subset L^{1}(Q)$. In particular for $X \in L^{\infty} \subset L^{2}$, we have (cf. (Kentia, 2015, Thm.3.7) and Prop. 2.5) that $\pi_{t}^{u}(X)=\operatorname{ess} \sup _{Q \in \overline{\mathcal{Q}^{\text {ngd }}}} E_{t}^{Q}[X]$, where

$$
\overline{\mathcal{Q}^{\text {ngd }}}:=\left\{Q \in \mathcal{M}^{e} \mid d Q / d P=\mathcal{E}(\lambda \cdot W), \lambda \text { predictable and } \lambda \in C\right\}
$$

is a larger set than $\mathcal{Q}^{\text {ngd }}$, containing measures with Girsanov kernels that are not necessarily bounded. In the definition (2.4) and in subsequent definitions of sets of equivalent measures, we tacitly assume (if not automatically satisfied) that Girsanov kernels $\lambda$ are such that stochastic exponentials $\mathcal{E}(\lambda \cdot W)$ are uniformly integrable martingales. We recall that for radial constraints $C$ (like in (3.4) with $A \equiv \operatorname{Id}_{\mathbb{R}^{n}}$ and constant $h \in(0, \infty))$, common in the good-deal literature, one has a known financial justification. By a direct duality argument, one can see (e.g. in a semimartingale framework, Becherer, 2009, Sect.3) that any (arbitrage-free) extension $\bar{S}=\left(S, S^{\prime}\right)$ of the market $S$ by derivative price processes $S^{\prime}:=E_{t}^{Q}[X]$ for contingent claims $X$ (with $Q \in \mathcal{M}^{e}, X^{-} \in L^{\infty}, X^{+} \in L^{1}(Q)$ ) does permit only for wealth processes $V>0$ of self-financing trading strategy (in $\bar{S}$ ) whose expected growth rates (log utilities) over any time period $0 \leq t<\tau \leq T$ satisfy the (sharp) estimate $E_{t}^{P}\left[\log \frac{V_{\tau}}{V_{t}}\right] \leq E_{t}^{P}\left[-\log \frac{Z_{\tau}}{Z_{t}}\right]$, where $Z$ is the density process of $Q$. For $Q \in \mathcal{Q}^{\text {ngd }}$ with radial constraint, this estimate is bounded by $h^{2}(\tau-t) / 2$, ensuring a bound $h^{2} / 2$ to expected growth rates (good deals) for any market extension (ideas going back at least to Cochrane and Requejo, 2000; Cerný and Hodges, 2002).

For the good-deal bounds to have nice dynamic properties, multiplicative stability (m-stability) of the set of no-good-deal measures is important. M-stability of dominated families of probability measures in dual representations (like e.g. (2.3)) for dynamic coherent risk measures (see e.g. Artzner et al., 2007) ensures in particular time consistency (recursiveness) and has been studied in a general context by Delbaen (2006). In economics, it is known as rectangularity (Chen and Epstein, 2002). A set $\mathcal{Q}$ of measures $Q \sim P$ is called m-stable if for all $Q^{1}, Q^{2} \in \mathcal{Q}$ with density processes $Z^{1}, Z^{2}$ and for all stopping times $\tau \leq T$, the process $Z:=I_{[0, \tau]} Z^{1}+I_{] \tau, T]} Z_{\tau}^{1} Z_{\cdot}^{2} / Z_{\tau}^{2}$ is the density process of a measure in 
$\mathcal{Q}$, where $[0, \tau]:=\{(t, \omega) \in[0, T] \times \Omega \mid t \leq \tau(\omega)\}$ denotes the stochastic interval and $I_{A}$ is the indicator function on a set $A$. As noted in (Delbaen, 2006, Rem. 6), by closure this definition extends to sets of measures that are absolutely continuous but not necessarily equivalent; such is formally achieved by setting $Z_{T}^{2} / Z_{\tau}^{2}=1$ on $\left\{Z_{\tau}^{2}=0\right\}$. The role of m-stability shows in results due to Delbaen (2006), stated in Lemma 2.1, Part a); for details cf. (Klöppel and Schweizer, 2007, Thm.2.7) or (Becherer, 2009, Prop.2.6). Proof for part b) is provided in the appendix.

Lemma 2.1. Let $\mathcal{Q}$ be a convex and m-stable set of probability measures $Q \sim P$ and $\pi_{t}^{u, \mathcal{Q}}(X):=$ ess $\sup _{Q \in \mathcal{Q}} E_{t}^{Q}[X]$, for $X \in L^{\infty}$.

a) There exists a càdlàg process $Y$ such that for all stopping times $\tau \leq T, Y_{\tau}=$ ess $\sup _{Q \in \mathcal{Q}} E_{\tau}^{Q}[X]=$ : $\pi_{\tau}^{u, \mathcal{Q}}(X)$. Moreover $\pi^{u, \mathcal{Q}}(\cdot)$ has the properties of a dynamic coherent risk measure. It is recursive and stopping time consistent: For stopping times $\sigma \leq \tau \leq T$ holds $\pi_{\sigma}^{u, \mathcal{Q}}\left(X^{1}\right)=\pi_{\sigma}^{u, \mathcal{Q}}\left(\pi_{\tau}^{u, \mathcal{Q}}\left(X^{1}\right)\right)$, and $\pi_{\tau}^{u, \mathcal{Q}}\left(X^{1}\right) \geq \pi_{\tau}^{u, \mathcal{Q}}\left(X^{2}\right)$ for $X^{1}, X^{2} \in L^{\infty}$ implies $\pi_{\sigma}^{u, \mathcal{Q}}\left(X^{1}\right) \geq \pi_{\sigma}^{u, \mathcal{Q}}\left(X^{2}\right)$. Finally, a supermartingale property holds: For all stopping times $\sigma \leq \tau \leq T$ and $Q \in \mathcal{Q}, \pi_{\sigma}^{u, \mathcal{Q}}(X) \geq E_{\sigma}^{Q}\left[\pi_{\tau}^{u, \mathcal{Q}}(X)\right]$, and $\pi^{u, \mathcal{Q}}(X)$ is a supermartingale under any $Q \in \mathcal{Q}$.

b) The sets $\mathcal{M}^{e}$ and $\mathcal{Q}^{\text {ngd }}$ are $m$-stable and convex and hence for $\mathcal{Q}=\mathcal{Q}^{\text {ngd }}, \pi^{u}(X)=\pi^{u, \mathcal{Q}}(X)$ satisfies the properties of Part a).

2.1. Parametrizations in an Itô process model. This section describes the Itô process framework for the financial market, and details the parametrizations for dynamic trading strategies and no-gooddeal constraints. The latter are specified at this stage by abstract correspondences (2.2) such that respective dynamic no-good-deal valuation bounds for contingent claims can be conveniently described in terms of (super-)solutions to BSDEs (Sections 2.2-2.3) within a convenient framework that is sufficiently general for all later Sections 3-4.

We consider models for financial markets where prices $\left(S^{i}\right)_{i=1 \ldots d}$ of $d$ risky assets evolve according to a stochastic differential equation (SDE)

$$
d S_{t}=\operatorname{diag}\left(S_{t}\right) \sigma_{t}\left(\xi_{t} d t+d W_{t}\right)=: \operatorname{diag}\left(S_{t}\right) \sigma_{t} d \widehat{W}_{t}, t \in[0, T], \quad S_{0} \in(0, \infty)^{d},
$$

for predictable $\mathbb{R}^{d}$ - and $\mathbb{R}^{d \times n}$-valued coefficients $\xi$ and $\sigma$, with $d \leq n$. This includes basically all examples of continuous price and state evolutions in (typically incomplete) markets of the good-deal literature, and permits also for non-Markovian evolutions. Risky asset prices $S$ are given in units of some riskless numéraire asset whose discounted price $S^{0} \equiv 1$ is constant. We assume that $\sigma$ is of maximal rank $d \leq n$ (i.e. $\operatorname{det}\left(\sigma_{t} \sigma_{t}^{\mathrm{tr}}\right) \neq 0$, that means no locally redundant assets) and that the market price of risk process $\xi$, satisfying $\xi_{t} \in \operatorname{Im} \sigma_{t}^{\mathrm{tr}}$, is bounded. This ensures that the market is free of arbitrage but typically incomplete (if $d<n$ ) in the sense that $\mathcal{M}^{e} \neq \emptyset$, as the minimal local martingale measure $\widehat{Q}$ given by $d \widehat{Q}=\mathcal{E}(-\xi \cdot W) d P$ (see Schweizer, 2001) is in $\mathcal{M}^{e}$, which however is typically not a singleton. Trading strategies are represented by the amount of wealth $\varphi=\left(\varphi_{t}^{i}\right)_{i}$ invested in the risky assets $\left(S^{i}\right)_{i}$. A self-financing trading strategy is described by a pair $\left(V_{0}, \varphi\right)$, where $V_{0}$ is the initial capital while $\varphi=\left(\varphi_{t}^{i}\right)_{i}$ describes the amount of wealth invested in the risky assets $\left(S^{i}\right)_{i}$ at any time $t$. The set $\Phi_{\varphi}$ of permitted strategies consists of $\mathbb{R}^{d}$-valued predictable processes $\varphi$ satisfying $E^{P}\left[\int_{0}^{T}\left|\varphi_{t}^{\operatorname{tr}} \sigma_{t}\right|^{2} d t\right]<\infty$. For a permitted strategy $\varphi$, the associated wealth process $V$ from initial capital $V_{0}$ has dynamics $d V_{t}=\varphi_{t}^{\operatorname{tr}} \sigma_{t} d \widehat{W}_{t}$. To ease notation, we re-parametrize strategies in $\Phi_{\varphi}$ in terms of integrands $\phi:=\sigma^{\operatorname{tr}} \varphi$ with respect to $\widehat{W}$. Indeed, equalities $\phi=\sigma^{\operatorname{tr}} \varphi$ and $\varphi=\left(\sigma^{\operatorname{tr}}\right)^{-1} \phi$, where $\left(\sigma^{\operatorname{tr}}\right)^{-1}:=\left(\sigma \sigma^{\operatorname{tr}}\right)^{-1} \sigma$ is the pseudo-inverse of $\sigma^{\operatorname{tr}}$, provide a one-to-one relation between $\varphi$ and $\phi$. Define the correspondences

$$
\Gamma_{t}(\omega):=\operatorname{Im} \sigma_{t}^{\operatorname{tr}}(\omega) \text { and } \Gamma_{t}^{\perp}(\omega):=\operatorname{Ker} \sigma_{t}(\omega), \quad(t, \omega) \in[0, T] \times \Omega,
$$

where $\operatorname{Im} \sigma_{t}^{\text {tr }}$ and Ker $\sigma_{t}$ denote the range (image) and the kernel of the respective matrices. Clearly, $\mathbb{R}^{n}=\Gamma_{t} \oplus \Gamma_{t}^{\perp}$ and any $z \in \mathbb{R}^{n}$ decomposes uniquely into its orthogonal projections as $z=\Pi_{\Gamma_{t}}(z) \oplus$ $\Pi_{\Gamma_{t}^{\perp}}(z)=: \Pi_{t}(z) \oplus \Pi_{t}^{\perp}(z)$. Let

$$
\Phi=\Phi_{\phi}:=\left\{\phi \mid \phi \text { is predictable, } \phi \in \Gamma \text { and } E\left[\int_{0}^{T}\left|\phi_{t}\right|^{2} d t\right]<\infty\right\}
$$

denote the (re-parametrized) set of permitted trading strategies. Proving the claims of the next proposition is routine (using Rockafellar (1976) for Part 1). 


\section{Proposition 2.2.}

(1) The correspondences $\Gamma, \Gamma^{\perp}$ are closed-convex-valued and predictable.

(2) $Q$ is in $\mathcal{M}^{e}$ if and only if $Q \sim P$ with $d Q=\mathcal{E}(\lambda \cdot W) d P$, where $\lambda$ is predictable and $\lambda=-\xi+\eta$, with $-\xi_{t}=\Pi_{t}\left(\lambda_{t}\right) \in \operatorname{Im} \sigma_{t}^{t r}$ and $\eta_{t}=\Pi_{t}^{\perp}\left(\lambda_{t}\right) \in \operatorname{Ker} \sigma_{t}, t \leq T$.

By Part 2 of Proposition 2.2, the set $\mathcal{Q}^{\text {ngd }}$ defined in (2.2) can be written as

$$
\mathcal{Q}^{\text {ngd }}=\{Q \sim P \mid d Q / d P=\mathcal{E}(\lambda \cdot W), \lambda \text { predictable, bounded and } \lambda \in \Lambda\},
$$

where $\Lambda:[0, T] \times \Omega \rightsquigarrow \mathbb{R}^{n}$ is defined by $\Lambda_{t}(\omega):=C_{t}(\omega) \cap\left(-\xi_{t}(\omega)+\operatorname{Ker} \sigma_{t}(\omega)\right)$. By Part 1 of Proposition 2.2 and (Rockafellar, 1976, Cor.1.K and Thm.1.M), $\Lambda$ is a compact-convex-valued predictable correspondence. Slightly beyond the no-free-lunch with vanishing risk condition, we assume that $\mathcal{Q}^{\text {ngd }}$ contains the measure $\widehat{Q}$, or equivalently $-\xi \in C$. This implies that $\Lambda$ is non-empty valued, hence standard.

2.2. Good-deal valuation with uniformly bounded correspondences. We here consider the case where the no-good-deal restriction is described by a uniformly bounded correspondence; a more general case is studied afterwards. We say that a correspondence $C$ is uniformly bounded if it satisfies

Assumption 2.3. $\sup _{(t, \omega)} \sup _{x \in C_{t}(\omega)}|x|<\infty$.

Let $C:[0, T] \times \Omega \rightsquigarrow \mathbb{R}^{n}$ be a standard correspondence satisfying Assumption 2.3 and $0 \in C$. Under Assumption 2.3, selections of $C$ are uniformly bounded processes. In particular, the Girsanov kernels of no-good-deal measures are uniformly bounded, and hence boundedness in the definition (2.2) (see also $(2.6))$ of $\mathcal{Q}^{\text {ngd }}$ is not necessary. The good-deal valuation bound $\pi_{t}^{u}(X):=\operatorname{ess} \sup _{Q \in \mathcal{Q}^{\text {ngd }}} E_{t}^{Q}[X]$ is well-defined for a contingent claim $X \in L^{2} \supset L^{\infty}$, that may be path-dependent, and one can check that in this case an analog of Part a) of Lemma 2.1 still holds. Though Assumption 2.3 fits well with the classical theory, it would be too restrictive to impose it in general since it may not hold in some interesting practical situations; see for instance the example in Section 3.2.3. Let us recall a fact about linear BSDEs (cf. El Karoui et al., 1997) which explains their role for valuation purposes.

Lemma 2.4. For $Q \sim P$ with bounded Girsanov kernel $\lambda$, the linear BSDE

$$
-d Y_{t}=Z_{t}^{t r} \lambda_{t} d t-Z_{t}^{t r} d W_{t}, t \leq T, \quad \text { with } \quad Y_{T}=X \text { in } L^{2}
$$

has a unique standard solution $\left(Y^{\lambda}, Z^{\lambda}\right)$ with $Y_{t}^{\lambda}=E_{t}^{Q}[X]=Y_{0}^{\lambda}+Z \cdot W_{t}^{Q}$, and $W^{Q}:=W-\int_{0}^{*} \lambda_{t} d t$. If $X \in L^{\infty}$ then $Y$ is bounded.

Boundedness of $\lambda$ in Lemma 2.4 clearly implies that the parameters of the BSDE (2.7) are standard. For unbounded $\lambda$, the classical BSDE theory no longer applies and one needs different results to characterize the good-deal bounds in terms of BSDEs. Under Assumption 2.3, $\Lambda$ is uniformly bounded and thus Girsanov kernels $\lambda^{Q}$ for all $Q \in \mathcal{Q}^{\text {ngd }}$ are bounded by the same constant. One has the following

Proposition 2.5. Let Assumption 2.3 hold. For $X \in L^{2}$ and $\lambda=\lambda^{Q} \in \Lambda, Q \in \mathcal{Q}^{\text {ngd }}$, let $\left(Y^{\lambda}, Z^{\lambda}\right)$ and $(Y, Z)$ be the standard solutions to the classical BSDEs (2.7) and

$$
-d Y_{t}=g\left(t, Z_{t}\right) d t-Z_{t}^{t r} d W_{t}, t \leq T, \text { and } \quad Y_{T}=X,
$$

with generator g given by $g(t, \cdot, z):=\sup _{\lambda \in \Lambda} \lambda_{t}^{\operatorname{tr}}(\cdot) z$ for $t \in[0, T], z \in \mathbb{R}^{n}$. Then:

(1) There exists $\bar{\lambda} \in \Lambda$ such that $g\left(t, Z_{t}\right)=\bar{\lambda}_{t}^{t r} Z_{t}, P \otimes d t$-a.e..

(2) $\pi_{t}^{u}(X)=$ ess $\sup _{Q \in \mathcal{Q}^{\text {ngd }}} E_{t}^{Q}[X]=E_{t}^{\bar{Q}}[X]=Y_{t}$ holds for $\bar{Q} \in \mathcal{Q}^{\text {ngd }}$ given by $d \bar{Q}=\mathcal{E}(\bar{\lambda} \cdot W) d P$, and $Y_{t}=\operatorname{ess}_{\sup }{ }_{\lambda \in \Lambda} Y_{t}^{\lambda}=Y_{t}^{\bar{\lambda}}, t \in[0, T]$.

Proof. Because $\Lambda$ is a predictable correspondence, by measurable maximum and measurable selection results (Rockafellar, 1976, Thms. 2.K and 1.C), for each $z \in \mathbb{R}^{n}$ the function $g(\cdot, \cdot, z)$ is predictable and there exists $\lambda^{z} \in \Lambda$ such that $g(t, \omega, z)=\lambda_{t}^{z}(\omega) z$ for all $(t, \omega)$. By Assumption 2.3, the generators of the BSDEs $(2.7),(2.8)$ are uniformly Lipschitz in $z$. Being a Carathéodory function, $g$ is $\mathcal{P} \otimes \mathcal{B}\left(\mathbb{R}^{n}\right)$ measurable. So the parameters (or data) for the BSDEs (2.7),(2.8) are indeed standard. Part 1 follows again by the measurable maximum and measurable selection theorems, since $Z$ is predictable. For Part 2 , the measure $\bar{Q}$ is in $\mathcal{Q}^{\text {ngd }}$ since $\bar{\lambda} \in \Lambda$. The remainder of Part 2 follows by existence, uniqueness and comparison results for classical BSDEs, cf. (El Karoui et al., 1997, Sect.2-3) 
2.3. Good-deal valuation with non-uniformly bounded correspondences. To relax the Assumption 2.3 of uniform boundedness, we now admit for a non-uniformly bounded standard correspondence $C$, with $0 \in C$, which satisfies

$$
\exists R \text { predictable with } \sup _{x \in C_{t}(\omega)}|x| \leq R_{t}(\omega)<\infty \forall(t, \omega) \text { and } \int_{0}^{T}\left|R_{t}\right|^{2} d t<\infty .
$$

It is relevant to look beyond Assumption 2.3, because examples of practical interest require to do so, see Section 3.2.3 where quasi-explicit formulas of good-deal bounds are obtained in a stochastic volatility model, with $C$ not being uniformly bounded but satisfying (2.9). Classical BSDE results do not apply as before to characterize good-deal bounds directly by standard BSDE solutions. Yet, one can still (Kentia, 2015, Thm.3.7) approximate $\pi_{.}^{u}(X)$ for $X \in L^{\infty}$ by solutions to classical BSDEs for suitable truncations of $C$, and prove that $\pi_{t}^{u}(X)$ coincides with the essential supremum over the larger set $\overline{\mathcal{Q}^{\text {ngd }}} \subseteq \mathcal{M}^{e}$ given in (2.4). Given (2.9), we show that $\pi^{u}(X)$ is the minimal supersolution of the BSDE (2.8), and that it is the minimal solution to (2.8) if a worst-case measure $\bar{Q}$ for $\pi_{0}^{u}(X)$ exists. Obviously, a maximizing $\bar{Q}$ may be attained rather in the larger set $\overline{\mathcal{Q}^{\text {ngd }}}$.

Let $g:[0, T] \times \Omega \times \mathbb{R}^{n} \rightarrow \mathbb{R}$ be the generator function defined by

$$
g(t, \cdot, z)=\sup _{\lambda \in \Lambda} \lambda_{t}^{\operatorname{tr}}(\cdot) z, \quad \text { for } t \in[0, T], z \in \mathbb{R}^{n} .
$$

By condition (2.9), the function $g$ above is finitely defined since it satisfies $|g(t, \omega, z)| \leq R_{t}(\omega)|z|<\infty$ for all $(t, \omega, z)$. Note for each $(t, \omega)$ that $g(t, \omega, \cdot)$ is Lipschitz-continuous since $\Lambda_{t}(\omega)$ is compact. In addition by measurable selection arguments analogous to those in the proof of Prop.2.5 it follows that $g$ is indeed $\mathcal{P} \otimes \mathcal{B}\left(\mathbb{R}^{n}\right)$-measurable. Since $g$ may not be uniformly Lipschitz if $C$ does not satisfy Assumption 2.3, then $\pi^{u}(X)$ cannot directly be characterized by classical BSDEs. But one can still obtain a characterization by the minimal supersolution to the BSDE with data $(g, X)$.

Definition 2.6. $(Y, Z, K)$ is a supersolution of the BSDE with parameters $(f, X)$ if

$$
-d Y_{t}=f\left(t, Y_{t}, Z_{t}\right) d t-Z_{t}^{t r} d W_{t}+d K_{t} \quad \text { for } t \leq T, \text { and } Y_{T}=X,
$$

with $K$ non-decreasing càdlàg adapted, $K_{0}=0$, and $\int_{0}^{T}\left|Z_{t}\right|^{2} d t<\infty$. A supersolution with $K \equiv 0$ is a BSDE solution. A (super)solution $(Y, Z, K)$ is minimal if $Y_{t} \leq \bar{Y}_{t}, t \in[0, T]$ holds for any other (super)solution $(\bar{Y}, \bar{Z}, \bar{K})$.

Note that a minimal supersolution when it exists is unique, as minimality implies uniqueness of the $Y$-components; since continuous local martingales of finite variation are trivial, identity of the $Z$ and $K$-components follows. Existence of the minimal supersolution is sometimes investigated under the condition that there exists at least one supersolution to the BSDE (cf. Drapeau et al., 2013). This condition is satisfied for the BSDE with parameters $(g, X), X \in L^{\infty}$ since $g(\cdot, 0)=0$ and thus $(Y, Z, K):=\left(|X|_{\infty}-\left(|X|_{\infty}-X\right) I_{\{T\}}, 0,\left(|X|_{\infty}-X\right) I_{\{T\}}\right)$ is a supersolution. Note that $g$ satisfies $g_{t}(z) \geq-\xi_{t}^{\operatorname{tr}} z, P \otimes d t$-a.e. and moreover $(g, X)$ satisfies the hypotheses of (Drapeau et al., 2013, Thm.4.17) which implies existence of the minimal supersolution to the BSDE with parameter $(g, X)$. We show that $\pi^{u}(X)$ can be identified with the $Y$-component of this minimal supersolution. Condition (2.9) ensures that the process $\int_{0}^{\cdot} g_{t}\left(Z_{t}\right) d t$ for $g$ in (2.10) and $Z$ satisfying $\int_{0}^{T}\left|Z_{t}\right|^{2} d t<\infty$ is real-valued, since the Cauchy-Schwarz inequality implies $\int_{0}^{T}\left|g_{t}\left(Z_{t}\right)\right| d t \leq\left(\int_{0}^{T}\left|Z_{t}\right|^{2} d t\right)^{\frac{1}{2}}\left(\int_{0}^{T}\left|R_{t}\right|^{2} d t\right)^{\frac{1}{2}}<\infty$.

Theorem 2.7. Let (2.9) hold and $X \in L^{\infty}$. There exists $Z \in \mathcal{H}^{2}(\widehat{Q})$ and a non-decreasing predictable process $K$ with $K_{0}=0$ such that $\left(\pi^{u}(X), Z, K\right)$ is the minimal supersolution to the BSDE for data $(g, X)$ with $g$ from (2.10), and $\pi^{u}(X) \in \mathcal{S}^{\infty}$.

The proof for this theorem is given in Appendix 5, while for details on the proof of the next corollary we refer to (Kentia, 2015, Corollary 3.10).

Corollary 2.8. Let (2.9) hold and $X \in L^{\infty}$. If there exists a measure $\bar{Q} \in \overline{\mathcal{Q}^{\text {ngd }}}$ such that $\pi_{0}^{u}(X)=$ $\sup _{Q \in \mathcal{Q}^{\text {ngd }}} E^{Q}[X]=E^{\bar{Q}}[X]$, then $\pi^{u}(X)$ is a $\bar{Q}$-martingale and there exists $Z \in \mathcal{H}^{2}(\widehat{Q})$ such that $\left(\pi^{u}(X), Z\right)$ is the minimal solution to the BSDE with parameters $(g, X)$ for $g$ defined in (2.10). The Girsanov kernel $\bar{\lambda}$ of $\bar{Q}$ satisfies ess $\sup _{\lambda \in \Lambda} \lambda_{t}^{\text {tr }} Z_{t}=\bar{\lambda}_{t}^{t r} Z_{t}, P \otimes d t$-a.e.. 
D. BECHERER AND K. KENTIA

In concrete cases, existence of $\bar{Q} \in \overline{\mathcal{Q}^{\text {ngd }}}$ as in Corollary 2.8 may be shown by direct considerations, see Section 3.2 for examples. If one specifies the no-good-deal restriction $(C)$ such that the set $\overline{\mathcal{Q}^{\text {ngd }}}$ becomes weakly compact in $L^{1}$, then $\bar{Q}$ would exist for any $X \in L^{\infty}$ as maximizer of a bounded linear functional over a weakly compact subset of $L^{1}$. Note that Assumption 2.3 only implies (by Dunford-Pettis theorem) that $\overline{\mathcal{Q}^{\text {ngd }}}$ is weakly relatively compact in $L^{1}$. Yet if $\mathcal{Q}^{\text {ngd }}$ is not weakly relatively compact in $L^{1}$, then by James' theorem (cf. Aliprantis and Border, 2006, Thm.6.36) there exists $X \in L^{\infty}$ such that the supremum in $\pi_{0}^{u}(X)=\sup _{Q \in \mathcal{Q}^{\text {ngd }}} E^{Q}[X]$ is not attained in the $L^{1}$-closure of $\overline{\mathcal{Q}^{\text {ngd }}}$ (note that $\overline{\mathcal{Q}^{\text {ngd }}}$ is convex), and in particular not in $\overline{\mathcal{Q}^{\text {ngd }}}$. Let us give an example where $\bar{Q}$ does not exist in $\overline{\mathcal{Q}^{\text {ngd }}}$ for some contingent claim and $C$ does neither satisfy Assumption 2.3 nor (2.9). Section 3.2.3 will furthermore give an example in a stochastic volatility model where $\bar{Q}$ exists and $C$ is not uniformly bounded but satisfies (2.9).

Example 2.9. Let $n=2$ with $W=\left(W^{1}, W^{2}\right), d=1$ with $d S_{t}=S_{t} \sigma^{S} d W_{t}^{1}, S_{0}>0, \sigma^{S}>0$, and $\xi=0$. Let $h>0$ be a deterministic predictable process with $\int_{0}^{T} h_{t} d t=\infty$ and $C_{t}(\omega):=\{0\} \times\left[-h_{t}, h_{t}\right],(t, \omega) \in$ $[0, T] \times \Omega$. Now let $X:=I_{\left\{W_{T}^{2} \geq 0\right\}} \in L^{\infty}$, then $\pi_{0}:=\sup _{n \in \mathbb{N}} Q^{n}\left[\left\{W_{T}^{2} \geq 0\right\}\right] \leq \pi_{0}^{u}(X) \leq 1$, where $d Q^{n}=\mathcal{E}\left(\lambda^{n} \cdot W^{2}\right) d P$ with $\lambda_{t}^{n}=h_{t} \wedge n, t \in[0, T], n \in \mathbb{N}$. The process $W^{2, n}:=W^{2}-\int_{0}^{\cdot} \lambda_{t}^{n} d t$ is a $Q^{n}$-Brownian motion. Hence $W_{T}^{2, n} \sim \mathcal{N}(0, T)$ under $Q^{n}$. We have $\int_{0}^{T} \lambda_{t}^{n} d t \nearrow \int_{0}^{T} h_{t} d t=\infty$ as $n \nearrow \infty$. Hence $\pi_{0}=\sup _{n \in \mathbb{N}} Q^{n}\left[\left\{W_{T}^{2, n} \geq-\int_{0}^{T} \lambda_{t}^{n} d t\right\}\right]=1$. Therefore $\pi_{0}^{u}(X)=1$. But there exists no measure $\bar{Q} \in \overline{\mathcal{Q}^{\text {ngd }}}$ such that $\pi_{0}^{u}(X)=E_{0}^{\bar{Q}}[X]$. Indeed for such a measure, one would have $\bar{Q}\left[\left\{W_{T}^{2} \geq 0\right\}\right]=1$ which is not possible since $\bar{Q} \sim P$.

\section{DyNAMic GOOD-DEAL HEDGing}

Let again $C$ be a standard correspondence satisfying $0 \in C$, and define the family of a-priori valuation measures

$$
\mathcal{P}^{\text {ngd }}:=\{Q \sim P \mid d Q / d P=\mathcal{E}(\lambda \cdot W), \lambda \text { predictable, bounded, } \lambda \in C\}
$$

which satisfy the same no-good-deal constraint as those in $\mathcal{Q}^{\text {ngd }}$, except that the local martingale condition for $S$ is omitted.

Remark 3.1. One could view $\mathcal{P}^{\text {ngd }}$ as the no-good-deal measures for a market consisting only of the riskless asset $S^{0} \equiv 1$, i.e. $\mathcal{P}^{\text {ngd }}=\mathcal{Q}^{\text {ngd }}(1)$. It is natural to define (3.1) as a-priori valuation measures, as the idea of no-good-deal valuation is to consider those risk neutral valuation measures $Q$, for which any extension of the financial market by additional derivatives' price processes (being Q-martingales) would not give rise to 'good deals'; see e.g. Björk and Slinko (2006); Klöppel and Schweizer (2007); Becherer (2009) for rigorous detail in continuous time for Sharpe ratios, utilities or growth rates; for concepts cf. (Cerný, 2003).

Like $\mathcal{Q}^{\text {ngd }}$, the set $\mathcal{P}^{\text {ngd }}$ clearly is again m-stable and convex. We define the a-priori dynamic coherent risk measure (in the sense of Lemma 2.1)

$$
\rho_{t}(X):=\underset{Q \in \mathcal{P} \text { ngd }}{\operatorname{ess} \sup _{t}} E_{t}^{Q}[X], \quad t \in[0, T]
$$

for suitable contingent claims $X$ for which $\rho_{t}(X)$ is finitely defined (e.g. $X$ bounded, or just in $L^{2}$ with $C$ satisfying Assumption 2.3). For a bounded correspondence $C$, one can describe $\rho(X)$ (analogous to $\pi^{u}(X)$ in Prop.2.5) by classical BSDEs:

Proposition 3.2. Let Assumption 2.3 hold. For $X \in L^{2}$, let $(\widetilde{Y}, \widetilde{Z})$ and $\left(Y^{\lambda}, Z^{\lambda}\right)$ (for $\left.\lambda \in C\right)$ be the respective standard solutions to the BSDEs

$$
\begin{aligned}
& -d Y_{t}=Z_{t}^{t r} \tilde{\lambda}_{t} d t-Z_{t}^{t r} d W_{t}, t \leq T, \text { with } Y_{T}=X, \quad \text { and } \\
& -d Y_{t}=Z_{t}^{t r} \lambda_{t} d t-Z_{t}^{t r} d W_{t}, t \leq T, \text { with } Y_{T}=X
\end{aligned}
$$

where $\tilde{\lambda} \in C$ is a predictable process satisfying $\tilde{\lambda}_{t}^{t r} Z_{t}=$ ess $\sup _{\lambda \in C} \lambda_{t}^{t r} Z_{t}, P \otimes d t$-a.e.. Then the measure $\widetilde{Q}$ with Girsanov kernel $\lambda^{\widetilde{Q}}=\tilde{\lambda}$ is in $\mathcal{P}^{\text {ngd }}$, and $\rho_{t}(X)=\operatorname{ess} \sup Y_{t}^{\lambda}=E_{t}^{\widetilde{Q}}[X]=\tilde{Y}_{t}, t \in[0, T]$. 
Elements $Q$ of $\mathcal{P}^{\text {ngd }}$ or $\mathcal{Q}^{\text {ngd }}$ could be seen as generalized scenarios (as in Artzner et al., 2007) for the dynamic coherent risk measures $\pi^{u}$ or $\rho$ (cf. Lemma 2.1). By $\mathcal{P}^{\text {ngd }} \cap \mathcal{M}^{e}=\mathcal{Q}^{\text {ngd }}$ one has $\rho_{t}(X) \geq \pi_{t}^{u}(X)$ for $t \leq T$. An investor holding a liability $X$ and trading in the market according to a permitted trading strategy $\phi$ would assign at time $t$ a residual risk $\rho_{t}\left(X-\int_{t}^{T} \phi_{s}^{\text {tr }} d \widehat{W}_{s}\right)$ to his position. The investor's objective is to hedge his position by a trading strategy $\bar{\phi}$ that minimizes his residual risk at any time $t \leq T$. To justify a premium $\pi^{u}(X)$ for selling $X$, the minimal capital requirement to make his position $\rho$-acceptable should coincide with $\pi^{u}(X)$. Thus, his hedging problem is to find a strategy $\bar{\phi} \in \Phi$ such that

$$
\pi_{t}^{u}(X)=\rho_{t}\left(X-\int_{t}^{T} \bar{\phi}_{s}^{\mathrm{tr}} d \widehat{W}_{s}\right)=\underset{\phi \in \Phi}{\operatorname{essinf}} \rho_{t}\left(X-\int_{t}^{T} \phi_{s}^{\mathrm{tr}} d \widehat{W}_{s}\right), \quad t \in[0, T] .
$$

The good-deal hedging strategy will be defined as a minimizer $\bar{\phi}$ in (3.3); If such a minimizer exists (as e.g. in Becherer (2009) or in later sections) the good-deal valuation $\pi^{u}(\cdot)$ coincides with the market consistent risk measure corresponding to $\rho$, in the spirit of Barrieu and El Karoui (2009). For contingent claim $X$, the tracking error $R_{t}^{\phi}(X)$ of a strategy $\phi \in \Phi$ is defined as the difference between the dynamic variations in the capital requirement and the profit/loss from trading (hedging) according to $\phi$, i.e. $R_{t}^{\phi}(X):=\pi_{t}^{u}(X)-\pi_{0}^{u}(X)-\phi \cdot \widehat{W}_{t}, t \in[0, T]$.

Proposition 3.3. Let $X \in L^{2}$ and $C$ satisfy Assumption 2.3. Then the tracking error $R^{\bar{\phi}}(X)$ for a strategy $\bar{\phi} \in \Phi$ solving (3.3) is a $Q$-supermartingale for all $Q \in \mathcal{P}^{\text {ngd }}$.

Proof. By the first equality of (3.3) and the definition of the tracking error one has $R_{t}^{\bar{\phi}}(X)=-\pi_{0}^{u}(X)+$ $\rho_{t}\left(X-\int_{0}^{T} \bar{\phi}_{s}^{\mathrm{tr}} d \widehat{W}_{s}\right), t \in[0, T]$. Note that $\int_{0}^{T} \bar{\phi}_{s}^{\mathrm{tr}} d \widehat{W}_{s} \in L^{2}(P)$ holds since $\xi$ is bounded and $\bar{\phi}$ is in $L^{2}(P \otimes d t)$. Now let $Q \in \mathcal{P}^{\text {ngd }}$ with $d Q / d P=\mathcal{E}\left(\lambda^{Q} \cdot W\right)$. By Proposition 3.2 this implies after a change of measures that $-d R_{t}^{\bar{\phi}}(X)=\left(\operatorname{ess}_{\sup _{\lambda \in C}} Z_{t}^{\mathrm{tr}} \lambda_{t}-Z_{t}^{\mathrm{tr}} \lambda_{t}^{Q}\right) d t-Z_{t}^{\mathrm{tr}} d W_{t}^{Q}, t \in[0, T]$. Because $R^{\bar{\phi}}(X) \in \mathcal{S}^{2}(P)$ and $d Q / d P \in L^{p}(P)$ for all $p<\infty$ (since $\lambda^{Q}$ is bounded), then Hölder's inequality implies that $R^{\bar{\phi}}(X) \in \mathcal{S}^{2-\epsilon}(Q)$ for $\epsilon \in(0,1)$. Furthermore under $Q$ the finite variation part of $R^{\bar{\phi}}(X)$ is non-increasing, and hence $R^{\bar{\phi}}(X)$ is a $Q$-supermartingale.

Remark 3.4. For a self-financing strategy $\phi \in \Phi$ replicating $X=x_{0}+\int_{0}^{T} \phi^{t r} d \widehat{W}$, with $x_{0} \in \mathbb{R}$, the tracking error vanishes, i.e. $R^{\phi}(X)=0$. One says that a strategy is mean-self-financing (like risk minimizing strategies studied in Schweizer (2001), Sect.2, with $E_{t}^{\widehat{Q}}[X]$ taking the role of $\left.\pi_{t}^{u}(X)\right)$ if its tracking error is a martingale (under P). Hence by Proposition 3.3, one can view the good-deal hedging strategy as being "at least mean-self-financing" under any $Q \in \mathcal{P}^{\text {ngd }}$, since its tracking error is a supermartingale under all measures in $\mathcal{P}^{\text {ngd }}$. Holding uniformly over all measures in $\mathcal{P}^{\text {ngd }}$, this could be seen as a robustness property of $\bar{\phi}$.

To describe solutions to the hedging problem (3.3), we will often assume that $C$ has further structure and is uniformly bounded. Section 3.2.3 also contains an example with a semi-explicit solution to the hedging problem in the Heston model for a correspondence $C$ that is not uniformly bounded but satisfies (2.9).

3.1. Results for ellipsoidal no-good-deal constraints. This section derives more explicit BSDE results to describe the solution to the valuation and the hedging problem (3.3) for (predictable) ellipsoidal no-good-deal constraints. This generalizes the important special case of radial constraints (as e.g. in Becherer, 2009), which is common to the good-deal literature and justified by bounds (uniform in $(t, \omega))$ on optimal growth rates or instantaneous Sharpe ratios, while still permitting comparably explicit results. The generalization could be interpreted as imposing different bounds on growth rates (or Sharpe ratios) for the risk factors associated to the principal axes. While such might appear as technical at this stage, in the subsequent context of model uncertainty (cf. Remark 4.7 b)) non-radial constraints will appear naturally.

To this end, let $h$ be a positive bounded predictable process, and $A$ be a predictable $\mathbb{R}^{n \times n}$-matrixvalued process with symmetric values and uniformly elliptic i.e. $A^{\text {tr }}=A$ and $x^{\text {tr }} A x \geq c|x|^{2}$ for all $x \in \mathbb{R}^{n}$, for some $c \in(0, \infty)$. The latter is a tractable and convenient (not necessarily most general) generalization of common good-deal constraints in the radial case, where $A \equiv \operatorname{Id}_{\mathbb{R}^{n}}$. It can be seen as 
setting constraints by scalar bounds on the Mahalanobis (1936)-distance of Girsanov kernels for the no-good-deal measures, instead of on their Euclidian norm.

We define the standard (see Rockafellar, 1976, Cor.1.Q) correspondence

$$
C_{t}(\omega)=\left\{x \in \mathbb{R}^{n} \mid x^{\mathrm{tr}} A_{t}(\omega) x \leq h_{t}^{2}(\omega)\right\}, \quad(t, \omega) \in[0, T] \times \Omega,
$$

that satisfies Assumption 2.3 due to ellipticity and boundedness of $h$. Assume that the kernel of the volatility matrix $\sigma$ is spanned by eigenvectors of $A$, i.e.

$$
A_{t}^{-1}\left(\operatorname{Ker} \sigma_{t}\right)=\operatorname{Ker} \sigma_{t}, \quad t \in[0, T] .
$$

As the eigenvectors of $A$ are orthogonal and $(\operatorname{Ker} \sigma)^{\perp}=\operatorname{Im} \sigma^{\operatorname{tr}}$, then (3.5) can be interpreted as separability of $\operatorname{Im} \sigma^{\operatorname{tr}}$ and Ker $\sigma$ in the sense that each of these subspaces has a basis of eigenvectors of $A$. Given (3.5), the subspaces $\operatorname{Im} \sigma^{\operatorname{tr}}$ and Ker $\sigma$ are orthogonal under the scalar product defined by $A$, one can re-write

$$
\mathcal{Q}^{\text {ngd }}=\left\{Q \sim P \mid d Q / d P=\mathcal{E}(\lambda \cdot W), \lambda \text { predictable, } \lambda=-\xi+\eta, \eta \in C^{\xi} \cap \text { Ker } \sigma\right\},
$$

with $C_{t}^{\xi}(\omega)=\left\{x \in \mathbb{R}^{n} \mid x^{\operatorname{tr}} A_{t}(\omega) x \leq h_{t}^{2}(\omega)-\xi_{t}(\omega)^{\operatorname{tr}} A_{t}(\omega) \xi_{t}(\omega)\right\}$, also satisfying Assumption 2.3. The correspondence $C^{\xi}$ is standard if

$$
h^{2}>\xi^{\operatorname{tr}} A \xi .
$$

The separability condition (3.5) ensures that $-\xi+\eta \in C$ is equivalent to $\eta \in C^{\xi}$, for $\eta \in \operatorname{Ker} \sigma$. This way the ellipsoidal constraint on the Girsanov kernels transfers to one on their $\eta$-component, which permits to formulate the no-good-deal restriction only with respect to non-traded risk factors in the market. In this setup, it is straightforward to obtain an expression for $\bar{\lambda}$ from Part 1 of Proposition 2.5 via

Lemma 3.5. For $z \in \mathbb{R}^{n} \backslash\{0\}, h>0$ and a symmetric positive definite $n \times n$-matrix $A$, the unique maximizer of $y^{t r} z$ subject to $y^{t r} A y \leq h^{2}$ is $\bar{y}=h\left(z^{t r} A^{-1} z\right)^{-1 / 2} A^{-1} z$.

For $X \in L^{2}$ with $C$ satisfying Assumption 2.3, the classical BSDE

$$
-d Y_{t}=\left(-\xi_{t}^{\mathrm{tr}} \Pi_{t}\left(Z_{t}\right)+\sqrt{h_{t}^{2}-\xi_{t}^{\mathrm{tr}} A_{t} \xi_{t}} \sqrt{\Pi_{t}^{\perp}\left(Z_{t}\right)^{\operatorname{tr}} A_{t}^{-1} \Pi_{t}^{\perp}\left(Z_{t}\right)}\right) d t-Z_{t}^{\mathrm{tr}} d W_{t}
$$

with terminal condition $Y_{T}=X$ has a unique standard solution $(Y, Z)$.

We will see that $\pi^{u}(X)=Y$ holds and that the optimal kernel $\bar{\lambda}$ from Proposition 2.5, Part 1, takes the form $\bar{\lambda}=-\xi+\bar{\eta}$, with $\bar{\eta} \in \operatorname{Ker} \sigma$ given by

$$
\bar{\eta}_{t}=\sqrt{\frac{h_{t}^{2}-\xi_{t}^{\mathrm{tr}} A_{t} \xi_{t}}{\Pi_{t}^{\perp}\left(Z_{t}\right)^{\mathrm{tr}} A_{t}^{-1} \Pi_{t}^{\perp}\left(Z_{t}\right)}} A_{t}^{-1} \Pi_{t}^{\perp}\left(Z_{t}\right), \quad t \in[0, T] .
$$

By Lemma 3.5 and (3.5), $\bar{\eta}_{t}^{\operatorname{tr}} \Pi_{t}^{\perp}\left(Z_{t}\right)=\operatorname{ess}_{\sup _{\eta \in C}{ }^{\xi} \cap \operatorname{Ker} \sigma .} \eta_{t}^{\operatorname{tr}} \Pi_{t}^{\perp}\left(Z_{t}\right), P \otimes d t$-a.e., thus $\bar{\lambda}_{t}^{\operatorname{tr}} Z_{t}=$ $-\xi_{t}^{\operatorname{tr}} \Pi_{t}\left(Z_{t}\right)+\left(h_{t}^{2}-\xi_{t}^{\operatorname{tr}} A_{t} \xi_{t}\right)^{1 / 2}\left(\Pi_{t}^{\perp}\left(Z_{t}\right)^{\operatorname{tr}} A_{t}^{-1} \Pi_{t}^{\perp}\left(Z_{t}\right)\right)^{1 / 2}, P \otimes d t$-a.e.. Therefore Part 2 of Proposition 2.5 yields

Theorem 3.6. Assume (3.5) and (3.6) hold. For $X \in L^{2}$, let $(Y, Z)$ be the standard solution to the $B S D E$ (3.7). Then $\pi_{t}^{u}(X)=Y_{t}=E_{t}^{\bar{Q}}[X], t \in[0, T]$, where $d \bar{Q}=\mathcal{E}((-\xi+\bar{\eta}) \cdot W) d P$ with $\bar{\eta}$ given explicitly by (3.8).

One can interpret the dynamics of the no-good-deal valuation (3.7) as follows. Writing it as $d Y_{t}=-a_{t} d t+\xi_{t}^{\operatorname{tr}} \Pi_{t}\left(Z_{t}\right) d t+Z_{t}^{\operatorname{tr}} d W_{t}=-a_{t} d t+\Pi_{t}\left(Z_{t}\right)^{\operatorname{tr}} d \widehat{W}_{t}+\Pi_{t}^{\perp}\left(Z_{t}\right)^{\operatorname{tr}} d \widehat{W}_{t}$ (cf. Section 2.1), with $a_{t}:=\sqrt{h_{t}^{2}-\xi_{t}^{\mathrm{tr}} A_{t} \xi_{t}} \sqrt{\Pi_{t}^{\perp}\left(Z_{t}\right)^{\mathrm{tr}} A_{t}^{-1} \Pi_{t}^{\perp}\left(Z_{t}\right)}$, it decomposes into a hedgeable part $\Pi_{t}\left(Z_{t}\right)^{\operatorname{tr}}\left(\xi_{t} d t+d W_{t}\right)=$ $\Pi_{t}\left(Z_{t}\right)^{\mathrm{tr}} d \widehat{W}$, that is dynamically spanned by tradeable assets, an orthogonal part $\Pi_{t}^{\perp}\left(Z_{t}\right)^{\mathrm{tr}} d \widehat{W}$, being a martingale under $P$ (and $\widehat{Q}$ ), and a premium part, where the rate $a_{t} \geq 0$ inherent to the upper good deal bound can be seen as compensation for the seller of the claim for non-tradeable risk. Note that $a>0$ on $\left\{(\omega, t): \Pi^{\perp}\left(Z_{t}\right) \neq 0\right\}$ by $(3.6)$.

The observation of the following lemma is straightforward.

Lemma 3.7. The matrices $A_{t}^{-1}(\omega)$, for $(t, \omega) \in[0, T] \times \Omega$, are positive-definite and satisfy $x^{t r} A_{t}^{-1}(\omega) x \geq$ $\alpha_{t}^{\prime}(\omega)|x|^{2}$ for all $x, t$, where $\alpha_{t}^{\prime}(\omega)=c\left\|A_{t}(\omega)\right\|^{-2}>0$ for $c$ being the constant of uniform ellipticity of A. Moreover $\|A\| \geq c$ holds. 
By Lemma 3.5 , the process $\tilde{\lambda}=h\left(Z^{\operatorname{tr}} A^{-1} Z\right)^{-1 / 2} A^{-1} Z$ satisfies $\tilde{\lambda}_{t}^{\operatorname{tr}} Z_{t}=\operatorname{ess}_{\sup _{\lambda^{\operatorname{tr}}} A_{\text {. }} \lambda_{.} \leq h^{2}} \lambda_{t}^{\operatorname{tr}} Z_{t}=$ $h_{t}\left(Z_{t}^{\operatorname{tr}} A_{t}^{-1} Z_{t}\right)^{1 / 2}, P \otimes d t$-a.e.. Hence Proposition 3.2 gives $\rho_{t}(X)=Y_{t}, t \in[0, T]$, where $(Y, Z)$ uniquely solves the classical BSDE with terminal condition $Y_{T}=X$ and

$$
-d Y_{t}=h_{t}\left(Z_{t}^{\mathrm{tr}} A_{t}^{-1} Z_{t}\right)^{1 / 2} d t-Z_{t}^{\mathrm{tr}} d W_{t} .
$$

Thanks to Lemma 3.7, a sufficient condition to ensure (3.6) is

$$
|\xi|<h \sqrt{\alpha^{\prime}} .
$$

In addition (3.10) is used to verify for Lemma 5.1 (stated in the appendix) the Kuhn-Tucker conditions before applying the Kuhn-Tucker theorem (see Rockafellar, 1970, Section 28), after which comparison results for BSDE yield the result of Theorem 3.8 below. The proof is omitted as it is analogous to that of (Becherer, 2009, Thm.5.4), using now Lemma 5.1 instead of Lem.6.1 therein. For $\phi \in \Phi$, let $\left(Y^{\phi}, Z^{\phi}\right)$ denote the standard solution to the BSDE with terminal condition $Y_{T}=X$ and, for $t \leq T$,

$$
-d Y_{t}=\left(-\xi_{t}^{\operatorname{tr}} \phi_{t}+h_{t}\left(\left(Z_{t}-\phi_{t}\right)^{\operatorname{tr}} A_{t}^{-1}\left(Z_{t}-\phi_{t}\right)\right)^{1 / 2}\right) d t-Z_{t}^{\operatorname{tr}} d W_{t} .
$$

Theorem 3.8. Assume (3.5),(3.10) hold. For $X \in L^{2}$, let $(Y, Z)$ and $\left(Y^{\phi}, Z^{\phi}\right)$ (for $\left.\phi \in \Phi\right)$ be standard solutions to the BSDEs (3.7),(3.11). Then $Y_{t}^{\phi}=\rho_{t}\left(X-\int_{t}^{T} \phi_{s}^{t r} d \widehat{W}_{s}\right), t \leq T$, and the strategy

$$
\bar{\phi}_{t}=\frac{\sqrt{\Pi_{t}^{\perp}\left(Z_{t}\right)^{t r} A_{t}^{-1} \Pi_{t}^{\perp}\left(Z_{t}\right)}}{\sqrt{h_{t}^{2}-\xi_{t}^{t r} A_{t} \xi_{t}}} A_{t} \xi_{t}+\Pi_{t}\left(Z_{t}\right)
$$

is in $\Phi$ and satisfies $Y_{t}^{\bar{\phi}}=\underset{\phi \in \Phi}{\operatorname{ess} \inf } Y_{t}^{\phi}=Y_{t}$ for any $t \in[0, T]$, that is

$$
\pi_{t}^{u}(X)=\underset{\phi \in \Phi}{\operatorname{essinf}} \rho_{t}\left(X-\int_{t}^{T} \phi_{s}^{t r} d \widehat{W}_{s}\right)=\rho_{t}\left(X-\int_{t}^{T} \bar{\phi}_{s}^{t r} d \widehat{W}_{s}\right)=Y_{t}^{\bar{\phi}} .
$$

Moreover, the tracking error $R^{\bar{\phi}}(X)$ is a supermartingale under all measures $Q \in \mathcal{P}^{\text {ngd }}$ and a martingale under the measure $Q^{\lambda} \in \mathcal{P}^{n g d}$ with Girsanov kernel $\lambda_{t}:=h_{t}\left(\left(Z_{t}-\bar{\phi}_{t}\right) A_{t}^{-1}\left(Z_{t}-\bar{\phi}_{t}\right)\right)^{-1 / 2} A_{t}^{-1}\left(Z_{t}-\bar{\phi}_{t}\right)$, $t \in[0, T]$.

The summands in the expression (3.12) for the strategy $\bar{\phi}$ play different roles from the perspective of hedging. The second summand is a non-speculative component that hedges locally tradeable risk by replication, while the first is a speculative component that compensates ("hedges") for unspanned non-tradeable risk by taking favorable bets on the market price of risk. Clearly, good deal bounds fit into the rich theory of $g$-expectations and market-consistent risk measures (cf. Barrieu and El Karoui, 2009, and more references therein). See Leitner (2007) for closely related ideas about instantaneous measurement of risk.

3.2. Examples for good-deal valuation and hedging with closed-form solutions. Explicit formulas, if available, facilitate intuition and enable fast computation of valuations, hedges and comparative statics. To this end, several concrete case studies are provided, starting with European options with monotone payoff profiles (e.g. call options) on non-traded assets in a multidimensional model of Black-Scholes type, in which tradeable assets only permit for partial hedging. In parallel to (Carassus and Temam, 2014, Prop.3, Sect.5.3) and Bayraktar and Young (2008), who employ SDE respectively PDE methods, this demonstrates how previous BSDE analysis can be applied in concrete case studies and we contribute some slight generalizations as well (e.g. higher dimensions, ellipsoidal constraints). As a further example, we contribute new explicit formulas for an option to exchange (geometric averages of) non-traded assets into traded assets. As before, the no-good-deal approach here gives rise to a familiar option pricing formula (by Margrabe) but suitable adjustments of parameter inputs are required, showing the difference to a simple no-arbitrage valuation approach that uses only one (given) single risk neutral measure. A further example derives semi-explicit good-deal solutions for the stochastic volatility model by Heston, for no-good-deal constraints on market prices of (unspanned) stochastic volatility risk which impose an interval range on the mean reversion level of the stochastic variance process under any valuation measure $Q \in Q^{\text {ngd }}$. Technically, this corresponds to imposing bounds on the instantaneous Sharpe ratio which are inversely proportional to the stochastic volatility. This is different to a related result by Bondarenko and Longarela (2009), in that their example imposes 
no good deal constraints in terms of bounds on simultaneous changes in the level of mean-reversion combined with opposite changes in reversion speed. We emphasize that, in addition to valuation formulas, all our examples provide explicit results for good-deal hedging strategies as well. Detailed derivations of the formulas in Sections 3.2.1-3.2.3 are given in Appendix 5

3.2.1. Closed-form formulas for options in a generalized Black-Scholes model. The market information $\mathbb{F}=\left(\mathcal{F}_{t}\right)_{t \geq 0}$ is generated by an $n$-dimensional $P$-Brownian motion $W:=\left(W^{1}, \ldots, W^{n}\right)^{\operatorname{tr}}$ with $W^{S}=$ $\left(W^{1}, \ldots, W^{d}\right)^{\operatorname{tr}}, d<n$ for $n, d \in \mathbb{N}$, and is augmented by null-sets. The financial market consists of $d \leq n$ (incomplete if $d<n$ ) stocks with (discounted) prices $S=\left(S^{k}\right)_{k=1}^{d}$ and further $n-d$ non-traded assets with values $H=\left(H^{l}\right)_{l=1}^{n-d}$. We consider a risk neutral model $\left(P=\widehat{Q} \in \mathcal{M}^{e}, \xi=0\right)$ where the processes $S$ and $H$ evolve as

$$
d S_{t}=\operatorname{diag}\left(S_{t}\right) \sigma^{S} d W_{t}^{S} \quad \text { and } \quad d H_{t}=\operatorname{diag}\left(H_{t}\right)\left(\gamma d t+\beta d W_{t}\right), \quad t \in[0, T],
$$

with $S_{0} \in(0, \infty)^{d}, H_{0} \in(0, \infty)^{n-d}$, constant coefficients $\sigma^{S}=\left(\sigma_{k i}^{S}\right)_{k, i} \in \mathbb{R}^{d \times d}$ invertible, $\beta=$ $\left(\beta_{l i}\right)_{l, i} \in \mathbb{R}^{(n-d) \times n}$ and $\gamma \in \mathbb{R}^{n-d}$. The volatility matrix of $S$ is $\sigma:=\left(\sigma^{S}, 0\right) \in \mathbb{R}^{d \times n}$ and is clearly of maximal rank $d \leq n$. For $z \in \mathbb{R}^{n}$, we have $\Pi(z)=\left(z^{1}, \ldots, z^{d}, 0, \ldots, 0\right)^{\operatorname{tr}} \in \mathbb{R}^{n}$ and $\Pi^{\perp}(z)=$ $\left(0, \ldots, 0, z^{d+1}, \ldots, z^{n}\right)^{\operatorname{tr}} \in \mathbb{R}^{n}$. We assume the ellipsoidal framework of Section 3.1, with $h \equiv$ const $>0$ and $A \equiv \operatorname{diag}(a)$, with $a \in(0, \infty)^{n}$. Clearly $A$ satisfies the assumption (3.5). From Theorem 3.6 we know that $\pi_{t}^{u}(X)=Y_{t}, t \in[0, T]$, for $(Y, Z)$ being the unique standard solution to the BSDE

$$
-d Y_{t}=h\left(\sum_{i=d+1}^{n}\left(Z_{t}^{i}\right)^{2} / a_{i}\right)^{1 / 2} d t-Z_{t}^{\mathrm{tr}} d W_{t}, t \leq T, \quad \text { and } \quad Y_{T}=X
$$

By Theorem 3.8 the good-deal hedging strategy is $\bar{\phi}_{t}=\Pi\left(Z_{t}\right) P \otimes d t$-a.e.. Define the geometric averages $\tilde{S}_{t}=\left(\prod_{k=1}^{d} S_{t}^{k}\right)^{1 / d}$ and $\tilde{H}_{t}=\left(\prod_{l=1}^{n-d} H_{t}^{l}\right)^{1 /(n-d)}$. One has $\tilde{S}_{t}=\tilde{S}_{0} \exp \left(\tilde{\sigma}^{\operatorname{tr}} W_{t}^{S}+\left(\tilde{\mu}-\frac{1}{2}|\tilde{\sigma}|^{2}\right) t\right)$ and $\tilde{H}_{t}=\tilde{H}_{0} \exp \left(\tilde{\beta}^{\operatorname{tr}} W_{t}+\left(\tilde{\gamma}-\frac{1}{2}|\tilde{\beta}|^{2}\right) t\right)$, where $\tilde{\sigma}:=\frac{1}{d}\left(\sigma^{S}\right)^{\operatorname{tr}} \mathbb{1}, \quad \tilde{\mu}:=\frac{1}{2}|\tilde{\sigma}|^{2}-\frac{1}{2 d}\left|\sigma^{S}\right|^{2}, \quad \tilde{\beta}=\frac{1}{n-d} \beta^{\operatorname{tr}} \mathbb{1}$ and $\tilde{\gamma}:=\frac{1}{n-d} \gamma^{\operatorname{tr}} \mathbb{1}+\frac{1}{2}|\tilde{\beta}|^{2}-\frac{1}{2(n-d)}|\beta|^{2}$, with $\mathbb{1}=(1, \ldots, 1)^{\operatorname{tr}}$. We treat the following two examples.

European option on non-traded assets: Consider a European option $X=G\left(\tilde{H}_{T}\right) \in L^{2}$ on the geometric average $\tilde{H}$, where the payoff $x \mapsto G(x)$ is non-decreasing, measurable and of polynomial growth in $x^{ \pm 1}$, i.e. $|G(x)| \leq k\left(1+x^{n}+x^{-n}\right)$ for all $x$ in $(0, \infty)$, for some $k>0$ and $n \in \mathbb{N}$. Let us consider the constant process $\lambda$ with value $h\left(\sum_{i=d+1}^{n} \tilde{\beta}_{i}^{2} / a_{i}\right)^{-1 / 2}\left(0, \ldots, 0, \tilde{\beta}_{d+1} / a_{d+1}, \ldots, \tilde{\beta}_{n} / a_{n}\right)^{\text {tr }}$ to identify the solution $(Y, Z)$ of the BSDE (3.13) as follows: We show that the standard solution $\left(Y^{\lambda}, Z^{\lambda}\right)$ to the linear BSDE (2.7) corresponding to $\lambda$ and with terminal condition $G\left(\tilde{H}_{T}\right)$ coincides with $(Y, Z)$. By Lemma 2.4 we know that $Y_{t}^{\lambda}=E_{t}^{Q^{\lambda}}\left[G\left(\tilde{H}_{T}\right)\right], t \in[0, T]$, with $d Q^{\lambda} / d P=\mathcal{E}\left(\lambda^{\operatorname{tr}} W\right)$. Moreover the Feynman-Kac formula yields $Y_{t}^{\lambda}=u^{\lambda}\left(t, \tilde{H}_{t}\right)$ and $Z_{t}^{\lambda}=\tilde{H}_{t} \partial_{x} u^{\lambda}\left(t, \tilde{H}_{t}\right) \tilde{\beta}$ for a function $u^{\lambda} \in \mathcal{C}^{1,2}([0, T) \times(0, \infty))$ solution to a Black-Scholes type PDE (after coordinate transformations that reduce the PDE into the heat equation (using Karatzas and Shreve, 2006, Sect.4.3)). Since $G$ is non-decreasing, then $\partial_{x} u^{\lambda} \geq 0$. Hence one has $P \otimes d t$-a.e. (omitting the argument $\left(t, \tilde{H}_{t}\right)$ of $\partial_{x} u^{\lambda}$ for simplicity)

$$
\lambda^{\operatorname{tr}} Z_{t}^{\lambda}=h \tilde{H}_{t} \partial_{x} u^{\lambda} \sqrt{\sum_{i=d+1}^{n} \frac{\tilde{\beta}_{i}^{2}}{a_{i}}}=h \sqrt{\sum_{i=d+1}^{n} \frac{\left(\tilde{H}_{t} \tilde{\beta}_{i} \partial_{x} u^{\lambda}\right)^{2}}{a_{i}}}=h \sqrt{\sum_{i=d+1}^{n} \frac{\left(Z_{t}^{\lambda, i}\right)^{2}}{a_{i}}},
$$

where the second equality uses $\partial_{x} u^{\lambda} \geq 0$. This implies that $\left(Y^{\lambda}, Z^{\lambda}\right)$ solves the BSDE (3.13), and therefore coincides with the latter's unique standard solution $(Y, Z)$. In particular we obtain $\pi_{t}^{u}(X)=Y_{t}^{\lambda}=E_{t}^{Q^{\lambda}}\left[G\left(\tilde{H}_{T}\right)\right], t \in[0, T]$. Moreover $\tilde{H}_{t}=\tilde{H}_{0} e^{\alpha_{+} t} \exp \left(\tilde{\beta}^{\operatorname{tr}} W_{t}^{\lambda}-\frac{1}{2}|\tilde{\beta}|^{2} t\right), t \in[0, T]$, where $\alpha_{ \pm}:=\tilde{\gamma} \pm h\left(\sum_{i=d+1}^{n} \tilde{\beta}_{i}^{2} / a_{i}\right)^{1 / 2}$ and $W^{\lambda}$ is an $n$-dimensional $Q^{\lambda}$-Brownian motion. Specifically for $G(x):=(x-K)^{+}, X$ is a call option on $\tilde{H}$ with strike $K$ and maturity $T$. Its upper good-deal bound at time $t \in[0, T]$ is therefore given by a Black-Scholes type formula (with "vol" abbreviating volatility)

$$
\begin{aligned}
& \pi_{t}^{u}(X)=N\left(d_{+}\right) \tilde{H}_{t} e^{\alpha_{+}(T-t)}-K N\left(d_{-}\right) \\
& \left.\quad=\text { B/S-call-price(time: } t, \text { spot: } \tilde{H}_{t} e^{\alpha_{+}(T-t)}, \text { strike: } K, \text { vol: }|\tilde{\beta}|\right)
\end{aligned}
$$


where $d_{ \pm}:=\left(\ln \left(\tilde{H}_{t} / K\right)+\left(\alpha_{+} \pm \frac{1}{2}|\tilde{\beta}|^{2}\right)(T-t)\right)(|\tilde{\beta}| \sqrt{T-t})^{-1}$ and $N$ is the cdf of the standard normal law. Analogously, the lower good-deal bound turns out as

$$
\left.\pi_{t}^{l}(X)=\mathrm{B} / \mathrm{S}-\text { call-price(time: } t \text {, spot: } \tilde{H}_{t} e^{\alpha_{-}(T-t)} \text {, strike: } K, \text { vol: }|\tilde{\beta}|\right) \text {. }
$$

The difference between the good-deal valuation formulas above and the standard Black-Scholes formula for risk-neutral valuation under measure $P=\widehat{Q}$ for a call option $\left(\tilde{H}_{T}-K\right)^{+}$shows by the additional factors $e^{\alpha_{ \pm}(T-t)}$ multiplying the spot price. This corresponds to an additional premium an option trader (selling at $\pi^{u}(X)$ or buying at $\pi^{l}(X)$ ) would require, if using the no-good-deal approach instead of simple arbitrage free valuation under a given risk neutral measure $P=\widehat{Q}$ (being an element of $\mathcal{Q}^{\text {ngd }}$ ). The hedging strategy for the seller of $X$ is

$$
\bar{\phi}_{t}=e^{\alpha_{+}(T-t)} N\left(d_{+}\right) \tilde{H}_{t}\left(\tilde{\beta}_{1}, \ldots, \tilde{\beta}_{d}, 0, \ldots, 0\right)^{\operatorname{tr}} \quad P \otimes d t \text { a.e. },
$$

which coincides with the delta (as computed under $P=\widehat{Q}$ ) for the call option only if $\alpha_{+}=0$. The hedging strategy of the buyer is derived analogously .

Exchange option of traded and non-traded assets: Consider an European option to exchange the traded asset $\tilde{S}$ for the non-traded asset $\tilde{H}$ at maturity $T$ with payoff $X=\left(\tilde{H}_{T}-\tilde{S}_{T}\right)^{+} \in L^{2}$. The upper bound $\pi_{t}^{u}(X)=E_{t}^{\bar{Q}}[X]$ can be explicitly derived using arguments from the previous example in combination with a change of numéraire (see Derivation of 3.33 in Section 3.4 of Kentia, 2015). We thereby obtain a Margrabe type formula

$$
\begin{aligned}
\pi_{t}^{u}(X) & =N\left(d_{+}\right) \tilde{H}_{t} e^{\alpha_{+}(T-t)}-N\left(d_{-}\right) \tilde{S}_{t} e^{\tilde{\mu}(T-t)} \\
& \left.=\text { B/S-call-price(time: } t, \text { spot: } \tilde{H}_{t} e^{\alpha_{+}(T-t)} \text {, strike: } \tilde{S}_{t} e^{\tilde{\mu}(T-t)} \text {, vol: } \delta\right),
\end{aligned}
$$

where $d_{ \pm}:=\left(\ln \left(\tilde{H}_{t} / \tilde{S}_{t}\right)+\left(\alpha_{+}+\tilde{\mu} \pm \frac{\delta^{2}}{2}\right)(T-t)\right)(\delta \sqrt{T-t})^{-1}$. Analogously, the corresponding lower good-deal bound is

$$
\left.\pi_{t}^{l}(X)=\mathrm{B} / \mathrm{S}-\text { call-price(time: } t \text {, spot: } \tilde{H}_{t} e^{\alpha_{-}(T-t)} \text {, strike: } \tilde{S}_{t} e^{\tilde{\mu}(T-t)} \text {, vol: } \delta\right) .
$$

The good-deal hedging strategy $\bar{\phi}_{t}$ for the seller of the exchange option equals

$$
N\left(d_{+}\right) \tilde{H}_{t} e^{\alpha_{+}(T-t)}\left(\tilde{\beta}_{1}, \ldots, \tilde{\beta}_{d}, 0, \ldots, 0\right)^{\operatorname{tr}}-N\left(d_{-}\right) \tilde{S}_{t} e^{\tilde{\mu}(T-t)}\left(\tilde{\sigma}_{1}, \ldots, \tilde{\sigma}_{d}, 0, \ldots, 0\right)^{\operatorname{tr}}
$$

$P \otimes d t$-a.e.. Again, the difference between the good-deal valuation formula and the classical Margrabe formula, as computed by standard no-arbitrage valuation under risk neutral measure $P=\widehat{Q}$, for the exchange option $\left(\tilde{H}_{T}-\tilde{S}_{T}\right)^{+}$shows by the presence of the factors involving the term $\alpha_{ \pm}$, which depends only on the parameters $A$ and $h$ for no-good-deal restrictions.

3.2.2. Computational results by Monte Carlo. To demonstrate that good-deal bounds and hedging strategies can be computed numerically in moderately high dimensions by generic simulation methods available for classical BSDE, we apply a (generic) multilevel Monte Carlo algorithm from Becherer and Turkedjiev (2014) (that builds on Gobet and Turkedjiev, 2016, reducing variances i.p. for $Z$ ) to approximate the solution $(Y, Z)$ of the $\operatorname{BSDE}(3.13)$ in dimension $n=4$, and compare with the known analytical solution for the exchange option $X:=\left(\tilde{H}_{T}-\tilde{S}_{T}\right)^{+}$. Using parameters $d=2, T=1$ and

$$
\begin{gathered}
H_{0}=\left(\begin{array}{l}
1 \\
1
\end{array}\right), \quad S_{0}=\left(\begin{array}{l}
1 \\
1
\end{array}\right), \quad \sigma^{S}=\left(\begin{array}{cc}
0.5 & 0.2 \\
0 & 0.4
\end{array}\right), \quad \gamma=(0.1,0.3)^{\mathrm{tr}}, \\
\beta=\left(\begin{array}{llll}
0.3 & 0.4 & 0.2 & 0.5 \\
0.5 & 0.7 & 0.3 & 0.4
\end{array}\right), \quad h=0.3, \quad \text { and } \quad A=\operatorname{diag}(0.5,0.65,0.8,0.95),
\end{gathered}
$$

we compare the approximate values at time $t=0$ to the known theoretical values obtained from Section 3.2.1. The exact value of the good-deal bound at time $t=0$ according to the formula (3.16) is then $\pi_{0}^{u}(X)=0.5494$, up to four digits, while for the hedging strategy it is $\bar{\phi}_{0}=(0.3049,0.4440,0,0)$, the exact value of $Z_{0}$ being $(0.3049,0.4440,0.2792,0.5025)$. We use a 4 -level algorithm on an equidistant time grid with $N=2^{4}$ steps, a number of sample paths $M=3 \times 10^{6}$ and with $K=50^{4}$ regression functions, being indicator functions on a hypercube partition of $\mathbb{R}^{4}$, the state space of the forward process $(S, H)$. Table 1 provides the numerical simulation results, summarized by the approximation means for the good-deal bound and the hedging strategy at time 0, the empirical root-mean-square errors (RMSE) computed coordinate-wise and the corresponding relative values (Rel.RMSE), based on 80 independent simulation runs. Simulation in Matlab for one run took $153 \mathrm{sec}$ on a core-i7 cpu laptop, 
showing relative errors (in terms of maximal coordinates in Rel.RMSE) of about $0.07 \%$ for valuation and $0.34 \%$ for hedging.

\begin{tabular}{|l|c|c|c|}
\cline { 2 - 4 } \multicolumn{1}{c|}{} & $Y_{0}$ approx & $Z_{0}$ approx & $\bar{\phi}_{0}$ approx \\
\hline Mean & 0.5499 & $(0.3052,0.4462,0.2852,0.5137)$ & $(0.3052,0.4462,0,0)$ \\
\hline RMSE & $10^{-4} \times 4$ & $10^{-4} \times(10,13,12,13)$ & $10^{-4} \times(10,13,0,0)$ \\
\hline Rel.RMSE & $10^{-4} \times 7$ & $10^{-4} \times(34,29,41,27)$ & $10^{-4} \times(34,29,0,0)$ \\
\hline
\end{tabular}

TABLE 1. Mean and (relative) root-mean-square errors of approximations

3.2.3. Semi-explicit formulas in the Heston stochastic volatility model. The market information is generated by a two-dimensional $P$-Brownian motion $W=\left(W^{S}, W^{\nu}\right)$, and is augmented by null-sets. We are going to consider a European put option $X=\left(K-S_{T}\right)^{+}$on $S$ with strike $K$ in the Heston model

$$
d S_{t}=S_{t} \sqrt{\nu_{t}} d W_{t}^{S} \quad \text { and } \quad d \nu_{t}=b\left(\frac{a}{b}-\nu_{t}\right) d t+\beta \sqrt{\nu_{t}}\left(\rho d W_{t}^{S}+\sqrt{1-\rho^{2}} d W_{t}^{\nu}\right), t \leq T,
$$

that is specified directly under a risk neutral measure $P=\widehat{Q}$, with $S_{0}, \nu_{0}>0, a, b, \beta>0$ and $\rho \in(-1,1)$. Here $b$ represents the mean-reversion speed, $a / b$ the mean-reversion level and $\beta / 2$ the volatility of the variance process $\nu$. Assume that the Feller condition $\beta^{2} \leq 2 a$ is satisfied, such that $\nu>0$. The equivalent local martingale measures $Q \in \mathcal{M}^{e}$ in this model are specified by Girsanov kernels $\lambda$ such that $d Q / d P=\mathcal{E}\left(\lambda \cdot W^{\nu}\right)$ is a uniformly integrable martingale. Indeed, we parametrize the pricing measures only by the second component of their Girsanov kernels (i.e. w.r.t. $W^{\nu}$ ) since the first component is always zero. We consider the no-good-deal constraint correspondence

$$
C_{t}(\omega)=\left\{x \in \mathbb{R}^{2}:|x| \leq \varepsilon / \sqrt{\nu_{t}(\omega)}\right\} \quad(t, \omega) \in[0, T] \times \Omega,
$$

for a constant $\varepsilon>0$. One observes that $C$ is standard with $0 \in C$, non-uniformly bounded and satisfies (2.9) for $R=\varepsilon / \sqrt{\nu}$ (since $\nu>0$ is continuous). Hence good-deal valuation results for uniformly bounded correspondences may not apply. We obtain a Heston-type formula (semi-explicit, computation requiring only 1-dim. integration) for the good-deal bound of the put option $X=\left(K-S_{T}\right)^{+}$,

$$
\left.\pi_{t}^{u}(X)=\text { Heston-put-price(time: } t, \bar{a}:=a+\beta \varepsilon \sqrt{1-\rho^{2}}, b, \beta\right),
$$

just like the ordinary Heston put price, associated to parameters $(t, a, b, \beta)$, but where the parameter $a$ has to be adjusted to $\bar{a}:=a+\beta \varepsilon \sqrt{1-\rho^{2}}$. The formula for the lower bound $\pi_{t}^{l}(X)$ is similar, but here $\bar{a}$ is replaced by $\underline{a}:=a-\beta \varepsilon \sqrt{1-\rho^{2}}$, for which the Feller condition still holds if $\varepsilon \leq \frac{1}{2} \beta^{-1}\left(2 a-\beta^{2}\right)\left(1-\rho^{2}\right)^{-1 / 2}$. In particular, $\pi_{t}^{u}(X)=E_{t}^{\bar{Q}}[X]$ holds with $d \bar{Q} / d P=\mathcal{E}\left((\varepsilon / \sqrt{\nu}) \cdot W^{\nu}\right)$. By Corollary 2.8 this yields $\bar{Y}=\pi^{u}(X)$ for the minimal solution $(\bar{Y}, \bar{Z}) \in \mathcal{S}^{\infty} \times \mathcal{H}^{2}$ of the BSDE

$$
-d Y_{t}=\frac{\varepsilon}{\sqrt{\nu_{t}}}\left|Z_{t}^{2}\right|-Z_{t}^{\mathrm{tr}} d W_{t}, t \in[0, T], \quad Y_{T}=\left(K-S_{T}\right)^{+} .
$$

The (seller's) good-deal hedging strategy $\bar{\phi}$ is given by the semi-explicit formula

$$
\bar{\phi}_{t}=S_{t} \sqrt{\nu_{t}} \Delta_{t}+\frac{\beta \rho}{2} \mathcal{V}_{t} \quad P \otimes d t \text {-a.e. }
$$

where $\Delta_{t}$ and $\mathcal{V}_{t}$ denote the delta and the vega of the put option at time $t$ in the Heston model with parameters $(\bar{a}, b, \beta)$. Derivations are provided in Appendix 5. We note that (3.20) coincides (cf. Poulsen et al., 2009) with the risk-minimizing strategy (in the sense of Schweizer, 2001) for the put in a Heston model, not with respect to the probability $P$ but with respect to the measure $\bar{Q}$ (derived just before) under which also Heston dynamics but with modified parameters prevail. This shows, how the strategy (3.20) differs from the standard risk minimizing strategy under $P$ (as in Poulsen et al., 2009; Heath et al., 2001). Good-deal valuation bounds for a put option in the Heston model are thus given by a Heston type formula but for a mean-reversion level increased by $\beta \varepsilon \sqrt{1-\rho^{2}} / b>0$. Similar to earlier examples, this difference constitutes an increase in the premium that an issuer selling at $\pi_{\text {. }}^{u}(X)$ would require according to good-deal valuation, in comparison to a standard arbitrage free valuation 
under one given risk neutral measure $P=\widehat{Q}$, when $S$ is the only risky asset available for hedging and stochastic volatility risk is otherwise taken to be unspanned. Figure 1 illustrates this, showing good deal valuations $\pi_{0}^{u}(X), \pi_{0}^{l}(X)$ (at $t=0$ ) for a long-dated put option with maturity $T=10$ in relation to the underlying $S_{0}$, for different no-good-deal constraint parameters $\varepsilon$. Other parameters are $K=100$, $a=0.12, b=3, \beta=0.3, \rho=-0.7, \nu_{0}=0.04$. The standard Heston price computed directly under a given risk neutral (minimal martingale) measure $P=\widehat{Q}$ (i.e. for $\varepsilon=0$ ) lies between the upper and lower good-deal bounds, whose spread increases with $\varepsilon>0$.

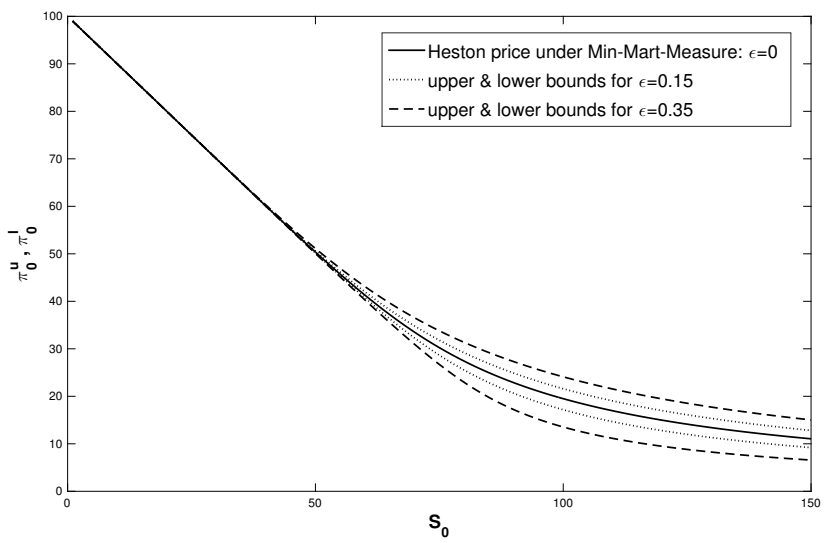

Figure 1. Dependence of $\pi_{0}^{u}(X), \pi_{0}^{l}(X)$ on $S_{0}$ and $\varepsilon$ in the Heston model for $K=100$, $a=0.12, b=3, \beta=0.3, \rho=-0.7, \nu_{0}=0.04, T=10$.

\section{Good-Deal Valuation AND hedging Under model UnCERTAinty}

In preceding sections, good-deal bounds and hedging strategies have been described by classical BSDEs under the probability measure $P$, expressing the objects of interest in terms of the market price of risk $\xi$ with respect to $P$. In reality, the objective real world probability measure is not precisely known, hence there is ambiguity about the market price of risk. To include model uncertainty (ambiguity) into the analysis, we follow a multiple priors approach in spirit of Gilboa and Schmeidler (1989); Chen and Epstein (2002); Epstein and Schneider (2003), by specifying a confidence region of reference probability measures $\left\{P^{\theta}: \theta \in \Theta\right\}$ (multiple priors, interpreted as potential real world probabilities of equal right), centered around some measure $P_{0}$. In practice, an investor facing model uncertainty may first extract an estimate $P_{0}$ for the true but uncertain $P$ from data, but then consider a class $\mathcal{R}$ of potential reference measures in some confidence region around $P_{0}$ to acknowledge the statistical uncertainty of estimation.

The starting point for the good-deal valuation approach under uncertainty is then to associate to each model $P^{\theta}$ its own family of (a-priori) no-good-deal measures $\mathcal{Q}^{\text {ngd }}\left(P^{\theta}\right)$ (resp. $\left.\mathcal{P}^{\text {ngd }}\left(P^{\theta}\right)\right)$. A robust worst-case approach requires the seller of a derivative to consider the (worst-case) model $P^{\bar{\theta}}$ that provides the largest upper good-deal valuation bound, to be conservative against model misspecification (see (4.21)). Such leads to wider good-deal bounds, corresponding to a larger overall set of no-good-deal measures under uncertainty. Notably, it will simultaneously also give rise to a suitable robust notion of good-deal hedging, which is uniform with respect to all $P^{\theta}$, by means of a saddle point result that ensures a minmax identity (see Theorem 4.13). We associate to each model $P^{\theta}$ a correspondence $C^{\theta}$ that defines the set of no-good-deal measures in this model. The aggregate set of no-good-deal measures will be described then by single correspondence $\widetilde{C}$, which incorporates also the uncertainty. Technically, this makes is possible to apply analysis obtained in the framework of previous sections, with $P_{0}$ taking the role of $P$.

Let us note that the worst-case and the multiple prior approach to uncertainty are classical in the theory, and so is the setup for our problem. Yet, they may be perceived as overly conservative, as results could be much influenced by just few extreme priors, what could appear even less desirable if one would not be ready to consider all priors to be of equal right. This may suggest directions for further research, e.g. to study some weighting or penalization of priors. It may also be interesting 
to study non-dominated priors (as e.g. in articles on superreplication under volatility uncertainty by 2nd-order BSDE) which do not fit within Section 4.

4.1. Model uncertainty framework. Let $\left(\Omega, \mathcal{F}, P_{0}, \mathbb{F}\right)$ be a probability space with a usual filtration $\mathbb{F}=\left(\mathcal{F}_{t}\right)_{t \leq T}$ generated by an $n$-dimensional $P_{0}$-Brownian motion $W^{0}$. We assume that all reference measures $P^{\theta}$ are equivalent to $P_{0}$ with corresponding Girsanov kernels $\theta$ evolving in some given confidence region $\Theta$. More precisely, we define

$$
\mathcal{R}:=\left\{P^{\theta} \sim P_{0} \mid d P^{\theta} / d P_{0}=\mathcal{E}\left(\theta \cdot W^{0}\right), \text { with } \theta \text { predictable and } \theta \in \Theta\right\},
$$

where $\Theta:[0, T] \times \Omega \rightsquigarrow \mathbb{R}^{n}$ is a standard correspondence satisfying Assumption 2.3 and $0 \in \Theta$, hence $P_{0} \in \mathcal{R} \neq \emptyset$. A similar framework has been considered for example in Chen and Epstein (2002); Quenez (2004) for solving the robust utility maximization problem under Knightian uncertainty about drift coefficients.

The financial market consists of $d \leq n$ tradeable risky assets whose discounted prices $\left(S^{i}\right)_{i=1}^{d}$ under $P^{\theta}($ for $\theta \in \Theta)$ evolve as Itô processes, solving the SDEs

$$
d S_{t}=\operatorname{diag}\left(S_{t}\right) \sigma_{t}\left(\xi_{t}^{\theta} d t+d W_{t}^{\theta}\right)=: \operatorname{diag}\left(S_{t}\right) \sigma_{t} d \widehat{W}_{t}^{\theta}, \quad t \leq T
$$

with $S_{0} \in(0, \infty)^{d}$, for $\mathbb{R}^{n}$-valued predictable $\xi^{\theta}$ and $\mathbb{R}^{d \times n}$-valued predictable volatility $\sigma$ of full rank, and $W^{\theta}:=W^{0}-\int_{0}^{\cdot} \theta_{s} d s$ a $P^{\theta}$-Brownian motion. Noting that market prices of risk, $\xi_{t}^{\theta}$ and $\xi_{t}^{0}$, canonically take values in $\operatorname{Im} \sigma_{t}^{\mathrm{tr}}$, we assume that market prices of risk $\xi^{\theta}$ (under $P^{\theta}$ for $\theta \in \Theta$ ) have the form

$$
\xi_{t}^{\theta}=\xi_{t}^{0}+\Pi_{t}\left(\theta_{t}\right) \in \operatorname{Im} \sigma_{t}^{\mathrm{tr}}, t \in[0, T]
$$

and that $\xi^{0}$ is bounded. By (4.2), the solutions of the SDEs (4.1) coincide $P_{0}$-a.s. for all $\theta \in \Theta$. The process $\xi^{\theta}$ (for $\theta \in \Theta$ ) is the market price of risk in the model $P^{\theta}$ and is also bounded (since $\xi^{0}$ is bounded and $\Theta$ satisfies Assumption 2.3). Hence, the minimal martingale measure (Schweizer, 2001) $\widehat{Q}^{\theta}$ with respect to $P^{\theta}$ is $d \widehat{Q}^{\theta}=\mathcal{E}\left(-\xi^{\theta} \cdot W^{\theta}\right) d P^{\theta}$. In addition $d \widehat{Q}^{\theta}=\mathcal{E}\left(\Pi^{\perp}(\theta) \cdot \widehat{W}^{0}\right) d \widehat{Q}^{0}$ and $\widehat{W}^{\theta}=\widehat{W}^{0}-\int_{0}^{\cdot} \Pi_{t}^{\perp}\left(\theta_{t}\right) d t$, for all $\theta \in \Theta$. We recall from Section 2.1 how dynamic trading strategies are defined and re-parametrized in terms of integrands $\left(\phi^{i}\right)_{i=1}^{d}$ with respect to $\widehat{W}^{0}$. The set of permitted trading strategies is

$$
\Phi:=\left\{\phi \mid \phi \text { is predictable, } \phi \in \operatorname{Im} \sigma^{\operatorname{tr}} \text { and } E^{P_{0}}\left[\int_{0}^{T}\left|\phi_{t}\right|^{2} d t\right]<\infty\right\} .
$$

Since $\phi^{\operatorname{tr}} \Pi^{\perp}(\theta)=0$ for $\theta \in \Theta$, the wealth process $V^{\phi}$ of strategy $\phi \in \Phi$ with initial capital $V_{0}$ is $V^{\phi}=V_{0}+\phi \cdot \widehat{W}^{\theta}=V_{0}+\phi \cdot \widehat{W}^{0}$, for all $\theta \in \Theta$. Let $\mathcal{M}^{e}\left(P^{\theta}\right):=\mathcal{M}^{e}\left(S, P^{\theta}\right)$ denote the set of equivalent local martingale measures for $S$ in the model $P^{\theta}$. Noting $P^{\theta} \sim P^{0}$ and recalling Proposition 2.2 one easily obtains

Proposition 4.1. $\mathcal{M}^{e}\left(P^{\theta}\right)=\mathcal{M}^{e}\left(P_{0}\right)$ for all $\theta \in \Theta$. In addition, every $Q \in \mathcal{M}^{e}\left(P^{\theta}\right)$ satisfies $d Q=\mathcal{E}\left(\lambda^{\theta} \cdot W^{\theta}\right) d P^{\theta}$ and $d Q=\mathcal{E}\left(\lambda^{0} \cdot W^{0}\right) d P_{0}$, with $\lambda^{\theta}=-\xi^{\theta}+\eta^{\theta}$ and $\lambda^{0}=-\xi^{0}+\eta^{0}$, where $\Pi^{\perp}\left(\lambda^{\theta}\right)=\eta^{\theta}, \quad \Pi^{\perp}\left(\lambda^{0}\right)=\eta^{0}$ and $\eta^{\theta}=\eta^{0}-\Pi^{\perp}(\theta)$.

Thus, we simply write $\mathcal{M}^{e}=\mathcal{M}^{e}(S)$ for the set of equivalent martingale measures.

4.2. No-good-deal restriction and good-deal bounds under uncertainty. Let $\left\{C^{\theta} \mid \theta \in \Theta\right\}$ be a family of standard correspondences satisfying

$$
-\xi^{\theta} \in C^{\theta} \quad \text { for all } \theta \in \Theta .
$$

In the model $P^{\theta}, \theta \in \Theta$, let the no-good-deal restriction be such that the Girsanov kernels of measures in $\mathcal{M}^{e}$ are selections of $C^{\theta}$. The resulting set $\mathcal{Q}^{\text {ngd }}\left(P^{\theta}\right)$ of no-good-deal measures is equal to

$$
\left\{Q \sim P^{\theta} \mid d Q / d P^{\theta}=\mathcal{E}\left(\lambda \cdot W^{\theta}\right), \lambda \text { predictable, bounded, } \lambda \in\left(-\xi^{\theta}+\operatorname{Ker} \sigma\right) \cap C^{\theta}\right\} .
$$

By (4.3), then $\widehat{Q}^{\theta} \in \mathcal{Q}^{\text {ngd }}\left(P^{\theta}\right) \neq \emptyset$ for all $\theta \in \Theta$. By Proposition 4.1 holds

$$
\mathcal{Q}^{\text {ngd }}\left(P^{\theta}\right)=\left\{Q \sim P_{0} \mid d Q / d P_{0}=\mathcal{E}\left(\lambda \cdot W^{0}\right), \quad \lambda \in-\xi^{0}+\left(\widetilde{C}^{\theta} \cap \operatorname{Ker} \sigma\right)\right\}
$$

where $\lambda$ is predictable and bounded, and for all $\theta \in \Theta$ the correspondences are

$$
\widetilde{C}^{\theta}:=C^{\theta}+\xi^{\theta}+\Pi^{\perp}(\theta)=C^{\theta}+\xi^{0}+\theta .
$$


Following a worst-case approach, we take the (robust) upper good-deal valuation $\pi^{u}(\cdot)$ under uncertainty as being the largest of all good-deal bounds $\pi^{u, \theta}(\cdot)$ over all models $P^{\theta}, \theta \in \Theta$. The respective set $\mathcal{Q}^{\text {ngd }}$ of no-good-deal valuation measures corresponding to $\pi^{u}(\cdot)$ can be described in terms of the sets $\mathcal{Q}^{\text {ngd }}\left(P^{\theta}\right), \theta \in \Theta$. At first, one might guess that $\mathcal{Q}^{\text {ngd }}$ should be the union of all $\mathcal{Q}^{\text {ngd }}\left(P^{\theta}\right)$. However, to have m-stability and convexity of $\mathcal{Q}^{\text {ngd }}$ for good dynamic properties of the resulting good-deal bounds (as in Lemma 2.1), one has to define $\mathcal{Q}^{\text {ngd }}$ as the smallest m-stable and convex set containing all $\mathcal{Q}^{\text {ngd }}\left(P^{\theta}\right), \theta \in \Theta$.

Definition 4.2. $\mathcal{Q}^{\text {ngd }}$ is the smallest m-stable convex subset of $\mathcal{M}^{e}$ containing all $\mathcal{Q}^{\text {ngd }}\left(P^{\theta}\right), \theta \in \Theta$. For sufficiently integrable claims $X$ (e.g. in $\left.L^{\infty}\right)$, the worst-case upper good-deal bound under uncertainty is $\pi_{t}^{u}(X):=$ ess $\sup _{Q \in \mathcal{Q}^{\text {ngd }}} E_{t}^{Q}[X]$.

We characterize the set $\mathcal{Q}^{\text {ngd }}$ from Definition 4.2 using a suitable single correspondence $\widetilde{C}$ which is derived from all $C^{\theta}, \theta \in \Theta$. To this end, we impose the

Assumption 4.3. The correspondence with values $\bigcup_{\theta \in \Theta} \widetilde{C}_{t}^{\theta}(\omega),(t, \omega) \in[0, T] \times \Omega$, is compact-valued and predictable.

The theory of measurable correspondences is well-developed for closed-valued correspondences (see Rockafellar, 1976). Assumption 4.3 ensures closed-valuedness and predictability of $\widetilde{C}$ for the proposition below. If all $C^{\theta}(\theta \in \Theta)$ are equal to some given $C^{0}$, as in the following example, such an assumption will automatically hold in the setting required for Section 4.4, where $\widetilde{C}^{\theta}(\theta \in \Theta)$ are ellipsoidal.

Example 4.4. For a standard correspondence $C^{0}$ with $\xi^{\theta} \in C^{0}, \theta \in \Theta$, let $C^{\theta}:=C^{0}, \theta \in \Theta$. Then $\widetilde{C}^{\theta}=C^{0}+\xi^{0}+\theta$ and $\bigcup_{\theta \in \Theta} \widetilde{C}^{\theta}=C^{0}+\xi^{0}+\Theta$ satisfies Assumption 4.3.

Proposition 4.5. Let Assumption 4.3 hold. Then $\mathcal{Q}^{\text {ngd }}$ equals

$$
\left\{Q \sim P_{0} \mid d Q / d P_{0}=\mathcal{E}\left(\lambda \cdot W^{0}\right), \lambda=-\xi^{0}+\eta \text { predictable, bounded, } \eta \in \widetilde{C}\right\},
$$

for the standard correspondence $\widetilde{C}_{t}(\omega):=\operatorname{Ker} \sigma_{t}(\omega) \cap \operatorname{Conv}\left(\bigcup_{\theta \in \Theta} \widetilde{C}_{t}^{\theta}(\omega)\right)$.

Proof. With Assumption 4.3, (Rockafellar, 1976, Thm.1.M and Prop.1.H) imply that $\widetilde{C}$ is standard. Note that $\widetilde{C}$ is non-empty-valued since $-\xi^{0} \in C^{0}$ and hence $0 \in \widetilde{C}_{t}^{0}(\omega) \cap \operatorname{Ker} \sigma_{t}(\omega) \subset \widetilde{C}_{t}(\omega)$. Denote by $\mathcal{Q}$ the set in (4.6). By definition $\widetilde{C}_{t}(\omega) \subset \operatorname{Ker} \sigma_{t}(\omega)$, implying $\mathcal{Q} \subseteq \mathcal{M}^{e}$. We first prove that $\mathcal{Q}^{\text {ngd }} \subseteq \mathcal{Q}$. Applying (Delbaen, 2006, Thm.1) or following the steps of the proof for Lemma 2.1, Part b), one sees that $\mathcal{Q}$ is m-stable and convex. By (4.4) and since $\widetilde{C}_{t}^{\theta}(\omega) \cap \operatorname{Ker} \sigma_{t}(\omega) \subseteq \widetilde{C}_{t}(\omega)$ for all $\theta \in \Theta$, then $\mathcal{Q}$ contains the union of all $\mathcal{Q}^{\text {ngd }}\left(P^{\theta}\right), \theta \in \Theta$. By definition $\mathcal{Q}^{\text {ngd }}$ is the smallest m-stable convex subset of $\mathcal{M}^{e}$ with this property, hence $\mathcal{Q}^{\text {ngd }} \subseteq \mathcal{Q}$.

Let us show $\mathcal{Q} \subseteq \mathcal{Q}^{\text {ngd }}$. The $L^{1}$-closure of $\mathcal{Q}^{\text {ngd }}$ is an m-stable closed and convex set of measures $Q \ll P_{0}$, and $\mathcal{Q}^{\text {ngd }}$ comprises exactly those elements of its closure that are equivalent to $P_{0}$. Closedness and convexity of the closure of $\mathcal{Q}^{\text {ngd }}$ are clear. We now show its m-stability. To this end, let $Z_{T}^{1}, Z_{T}^{2}$ be in the closure of $\mathcal{Q}^{\text {ngd }}, \tau \leq T$ be a stopping time and $Z_{T}:=Z_{\tau}^{1} Z_{T}^{2} / Z_{\tau}^{2} I_{\left\{Z_{\tau}^{2}>0\right\}}+Z_{\tau}^{1} I_{\left\{Z_{\tau}^{2}=0\right\}}$. There exist $\left(Z_{T}^{1, n}\right)_{n},\left(Z_{T}^{2, n}\right)_{n} \subseteq \mathcal{Q}^{\text {ngd }}$ such that $Z_{T}^{1, n} \rightarrow Z_{T}^{1}$ and $Z_{T}^{2, n} \rightarrow Z_{T}^{2}$ in $L^{1}$. By m-stability of $\mathcal{Q}^{\text {ngd }}$ holds $Z_{T}^{n}:=Z_{\tau}^{1, n} Z_{T}^{2, n} / Z_{\tau}^{2, n} \in \mathcal{Q}^{\text {ngd }}$ for each $n \in \mathbb{N}$. Now $E\left[Z_{T}^{n}\right]=1$ for all $n \in \mathbb{N}$, and $Z_{T}^{n} \rightarrow Z_{T}$ in probability as $n \rightarrow \infty$. In addition,

$$
\begin{aligned}
E\left[Z_{T}\right] & =E\left[Z_{\tau}^{1} Z_{T}^{2} / Z_{\tau}^{2} I_{\left\{Z_{\tau}^{2}>0\right\}}\right]+E\left[Z_{\tau}^{1} I_{\left\{Z_{\tau}^{2}=0\right\}}\right] \\
& =E\left[E_{\tau}\left[Z_{T}^{2} / Z_{\tau}^{2}\right] Z_{\tau}^{1} I_{\left\{Z_{\tau}^{2}>0\right\}}\right]+E\left[Z_{\tau}^{1} I_{\left\{Z_{\tau}^{2}=0\right\}}\right]=E\left[Z_{\tau}^{1}\right]=1 .
\end{aligned}
$$

By Scheffé's lemma one obtains $Z_{T}^{n} \rightarrow Z_{T}$ in $L^{1}$ as $n \rightarrow \infty$, and m-stability of the closure of $\mathcal{Q}^{\text {ngd }}$ follows. As $W^{0}$ is a continuous $P_{0}$-martingale with the predictable representation property, it satisfies the hypotheses of (Delbaen, 2006, Thm.2), implying by Definition 4.2 the existence of a closed-convex-valued predictable correspondence $C^{1}$ such that the no-good-deal measure set $\mathcal{Q}^{\text {ngd }}$ is equal to

$$
\left\{Q \sim P_{0} \mid d Q / d P_{0}=\mathcal{E}\left(\lambda \cdot W^{0}\right), \lambda=-\xi^{0}+\eta \text { predictable, } \eta \in C^{1} \cap \operatorname{Ker} \sigma\right\} .
$$

To prove the claim, it suffices to show that all predictable selections of $\widetilde{C}$ are also predictable selections of $C^{1} \cap \operatorname{Ker} \sigma$. To this end it suffices to show that for all $\theta \in \Theta$, any predictable selection of $\widetilde{C}^{\theta} \cap \operatorname{Ker} \sigma$ 
is a predictable selection of $C^{1} \cap \operatorname{Ker} \sigma$. Assume the contrary that there exists $\theta \in \Theta$ and a predictable process $\eta$ such that $\eta \in \widetilde{C}^{\theta} \cap \operatorname{Ker} \sigma$ and $\eta$ is not selection of $C^{1} \cap \operatorname{Ker} \sigma$. Then $\mathcal{E}\left(\left(-\xi^{0}+\eta\right) \cdot W^{0}\right)$ is in $\mathcal{Q}^{\text {ngd }}\left(P^{\theta}\right)$ but not in $\mathcal{Q}^{\text {ngd }}$, which contradicts $\mathcal{Q}^{\text {ngd }}\left(P^{\theta}\right) \subseteq \mathcal{Q}^{\text {ngd }}$.

Using the characterization of $\mathcal{Q}^{\text {ngd }}$ in Proposition 4.5 we can apply the results of Sections $2-3$ in order to derive worst-case good-deal bounds and hedging strategies under uncertainty like in the absence of uncertainty, with the center $P_{0}$ of the set of reference measures $\mathcal{R}$ taking the role of $P$ (in Sections 2-3) and the enlarged correspondence $\widetilde{C}$ taking the role of $C$ there.

Example 4.6. For $C^{\theta}, \theta \in \Theta$, as in Example 4.4 holds $\widetilde{C}=\left(C^{0}+\xi^{0}+\Theta\right) \cap \operatorname{Ker} \sigma$ and

$$
\mathcal{Q}^{\text {ngd }}=\left\{Q \sim P_{0} \mid d Q / d P_{0}=\mathcal{E}\left(\lambda \cdot W^{0}\right), \lambda \in\left(-\xi^{0}+\operatorname{Ker} \sigma\right) \cap\left(C^{0}+\Theta\right)\right\}
$$

with $\lambda$ denoting bounded predictable selections, by Proposition 4.5. Moreover the union $\bigcup_{\theta \in \Theta} \mathcal{Q}^{\text {ngd }}\left(P^{\theta}\right)$ is convex, $m$-stable (cf. Lemma 4.9) and equals $\mathcal{Q}^{\text {ngd }}$.

Remark 4.7. a) Equation (4.6) shows, how the good-deal valuation and hedging problem under model uncertainty can technically be embedded into the mathematical framework of Sections 2-3 without uncertainty, by considering an enlarged no-good-deal constraint correspondence $C$ as $\operatorname{Conv}\left(\cup_{\theta \in \Theta}\left(C^{\theta}+\theta\right)\right)$ in (2.6) with $P_{0}$ taking the role of $P$. In Example 4.6, (4.7), it simply means to take $C$ as $C^{0}+\Theta$.

b) Typical examples for good-deal constraints are radial, i.e. $C^{0}$ is a ball. This case is predominant in the literature and justified from a finance point of view by ensuring a constant bound on instantaneous Sharpe ratios (or growth rates). But typical examples for uncertainty (ambiguity) constraints $\Theta$ can well be non-radial (see Chen and Epstein, 2002; Epstein and Schneider, 2003). For instance, $\Theta$ may arise from a confidence region for some unknown drift parameters in a multivariate (log-)normal model; such would in general be ellipsoidal but not radial, and the sum $C^{0}+\Theta$ can even be non-ellipsoidal. To offer a suitable framework for such and other examples, Section 2 treats abstract correspondences. A constructive method to solve for such a typical parametrization of $C^{0}+\Theta$ is described in Remark 4.14.

4.3. Robust approach to good-deal hedging under model uncertainty. As in Section 3 (cf. (3.1) and the definition of $\mathcal{Q}^{\text {ngd }}\left(P^{\theta}\right)$ ), we define for $\theta \in \Theta$ the set

$$
\mathcal{P}^{\text {ngd }}\left(P^{\theta}\right):=\left\{Q \sim P^{\theta} \mid d Q / d P^{\theta}=\mathcal{E}\left(\lambda \cdot W^{\theta}\right), \lambda \in C^{\theta} \text { predictable, bounded }\right\}
$$

in order to introduce a robust notion of good-deal hedging. Let $\mathcal{P}^{\text {ngd }}$ be the smallest m-stable convex set of measures $Q \sim P_{0}$ containing all $\mathcal{P}^{\text {ngd }}\left(P^{\theta}\right), \theta \in \Theta$. Then

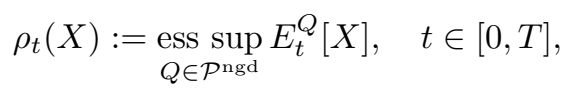

defines a time-consistent dynamic coherent risk measure by Lemma 2.1 (for $X \in L^{2}\left(P_{0}\right)$ if the correspondence from Assumption 4.3 is uniformly bounded, or for $X \in L^{\infty}$ otherwise). As in Section 3, the task of good-deal hedging under uncertainty is posed as a minimization problem (4.8) for a-priori risk measures $\rho$ of hedging errors: For a contingent claim $X$, find a strategy $\phi^{*} \in \Phi$ such that

$$
\left.\pi_{t}^{u}(X)=\rho_{t}\left(X-\int_{t}^{T} \phi_{s}^{* \operatorname{tr}} d \widehat{W}_{s}^{0}\right)=\underset{\phi \in \Phi}{\operatorname{essinf}} \rho_{t}\left(X-\int_{t}^{T} \phi_{s}^{\operatorname{tr}} d \widehat{W}_{s}^{0}\right)\right], \quad t \in[0, T] .
$$

The good-deal hedging strategy under uncertainty is defined as this minimizer (if it exists) $\phi^{*} \in \Phi$. For $X \in L^{2}\left(P_{0}\right)$, one can prove (as in Prop.3.3) that the tracking error $R^{\phi^{*}}(X)$ of the strategy $\phi^{*}$ is a supermartingale under every measure in $\mathcal{P}^{\text {ngd }}$ :

Proposition 4.8. Let $X \in L^{2}\left(P_{0}\right)$ with the correspondence defined in Assumption 4.3 being uniformly bounded. Then the tracking error $R^{\phi^{*}}(X)$ of a strategy $\phi^{*}$ solving (4.8) is a $Q$-supermartingale for all $Q \in \mathcal{P}^{n g d}$.

A strategy solving the good-deal hedging problem under uncertainty and whose tracking error satisfies the supermartingale property under all measures in $\mathcal{P}^{\text {ngd }}$ (as in Proposition 4.8) will be qualified as robust with respect to uncertainty. Note that this is a different notion of robustness compared to the one in Remark 3.4, because the supermartingale property has to hold for measures in $\mathcal{P}^{\text {ngd }}\left(P^{\theta}\right)$ uniformly for all models $P^{\theta} \in \mathcal{R}$ ( since $\bigcup_{\theta \in \Theta} \mathcal{P}^{\text {ngd }}\left(P^{\theta}\right)$ is a subset of $\mathcal{P}^{\text {ngd }}$ ). More concrete results under uncertainty will be derived next under additional conditions. 
4.4. Hedging under model uncertainty for ellipsoidal good-deal constraints. In this section we consider ellipsoidal good-deal constraints. To this end, let

$$
C_{t}^{0}(\omega)=\left\{x \in \mathbb{R}^{n} \mid x^{\operatorname{tr}} A_{t}(\omega) x \leq h_{t}^{2}(\omega)\right\}, \quad(t, \omega) \in[0, T] \times \Omega,
$$

where $A$ is a uniformly elliptic and predictable matrix-valued process, and $h$ some positive bounded and predictable process. We assume that $A$ satisfies the separability condition (3.5) with respect to $\sigma$. Let $\Theta$ be an arbitrary standard correspondence satisfying the uniform boundedness Assumption 2.3 and $0 \in \Theta$. As in Example 4.4, we let $C^{\theta}:=C^{0}$, for all $\theta \in \Theta$, yielding by (4.5) that

$$
\widetilde{C}_{t}^{\theta} \cap \operatorname{Ker} \sigma_{t}=\left\{x \in \mathbb{R}^{n} \mid x^{\operatorname{tr}} A_{t} x \leq h_{t}^{2}-\xi_{t}^{\theta^{\operatorname{tr}}} A_{t} \xi_{t}^{\theta}\right\} \bigcap \operatorname{Ker} \sigma_{t}+\Pi_{t}^{\perp}\left(\theta_{t}\right)
$$

Clearly, $C^{\theta}$ is standard and satisfies Assumption 2.3 for $\theta \in \Theta$. Similarly to (3.10), to derive explicit BSDE formulations for solving the hedging problem we will assume

$$
\left|\xi^{\theta}\right|<h \sqrt{\alpha^{\prime}} \text { for all } \theta \in \Theta,
$$

where the process $\alpha^{\prime}$ is the constant of ellipticity of $A^{-1}$ as in Lemma 3.7. Recall that, thanks to Lemma 3.7, the inequality (4.10) implies in particular that $-\xi^{\theta} \in C^{0}, \theta \in \Theta$; hence (4.3) holds and the correspondences $\widetilde{C}^{\theta} \cap$ Ker $\sigma$ are standard, $\theta \in \Theta$. Note that condition (4.10) ensures applicability of Lemma 5.1 in our current setup for any model $P^{\theta}$. Since $C^{\theta}$ is equal to $C^{0}$ and satisfies Assumption 2.3 , one has

$$
\mathcal{P}^{\text {ngd }}\left(P^{\theta}\right)=\left\{Q \sim P_{0} \mid d Q / d P_{0}=\mathcal{E}\left(\lambda \cdot W^{0}\right), \lambda \text { predictable, } \lambda \in C^{0}+\theta\right\} .
$$

The following lemma can be shown similarly to the proof of part b) of Lemma 2.1 using (4.11). For details see (Kentia, 2015, Lemma 3.26 and its proof).

\section{Lemma 4.9.}

(1) The set $\bigcup_{\theta \in \Theta} \mathcal{P}^{\text {ngd }}\left(P^{\theta}\right)$ is m-stable, convex and equal to $\mathcal{P}^{\text {ngd }}$.

(2) The set $\bigcup_{\theta \in \Theta} \mathcal{Q}^{\text {ngd }}\left(P^{\theta}\right)$ is m-stable, convex and equal to $\mathcal{Q}^{\text {ngd }}$.

Thanks to Lemma 4.9, the dynamic risk measure $\rho$ satisfies for $X \in L^{2}\left(P_{0}\right)$

$$
\rho_{t}(X):=\underset{Q \in \mathcal{P}^{\text {ngd }}}{\operatorname{ess} \sup _{t}} E_{t}^{Q}[X]=\underset{\theta \in \Theta}{\operatorname{ess} \sup } \rho_{t}^{\theta}(X), \quad t \in[0, T]
$$

with $\rho_{t}^{\theta}(X):=\operatorname{ess} \sup _{Q \in \mathcal{P}^{\text {ngd }}\left(P^{\theta}\right)} E_{t}^{Q}[X]$. The worst-case upper good-deal bound $\pi_{t}^{u}(X)$ for $X \in L^{2}\left(P_{0}\right)$ rewrites from Definition 4.2 as

$$
\pi_{t}^{u}(X):=\operatorname{ess} \sup _{\theta \in \Theta} \operatorname{ess~sup}_{Q \in \mathcal{Q}^{\text {ngd }}\left(P^{\theta}\right)} E_{t}^{Q}[X]=\underset{\theta \in \Theta}{\operatorname{ess} \sup } \pi_{t}^{u, \theta}(X), t \in[0, T]
$$

where $\pi_{t}^{u, \theta}(X)=\operatorname{ess} \sup _{Q \in \mathcal{Q}^{\text {ngd }}\left(P^{\theta}\right)} E_{t}^{Q}[X]$. The corresponding lower bound $\pi^{l}(X)$ is obtained via $\pi^{l}(X)=-\pi^{u}(-X)$. For a worst-case approach to uncertainty we will investigate valuation of claims according to $\pi^{u}(\cdot)$ and hedging with the optimal trading strategy solution to (4.8). We employ results from Section 3.1 (under $P=P^{\theta}$ ) to characterize $\pi^{u, \theta}(X)$ as well as the associated hedging strategies $\bar{\phi}^{\theta}$ in $\Phi$. For $\theta \in \Theta$ and $\phi \in \Phi$ let us consider the classical BSDEs

$$
\begin{aligned}
& -d Y_{t}=f^{\phi, \theta}\left(t, Z_{t}\right) d t-Z_{t}^{\mathrm{tr}} d W_{t}^{0}, \quad t \leq T, \quad Y_{T}=X, \\
& -d Y_{t}=f^{\theta}\left(t, Z_{t}\right) d t-Z_{t}^{\mathrm{tr}} d W_{t}^{0}, \quad t \leq T, \quad Y_{T}=X,
\end{aligned}
$$

with respective generators

$$
\begin{aligned}
f^{\phi, \theta}(t, z):= & \theta_{t}^{\operatorname{tr}}\left(z-\phi_{t}\right)-\xi_{t}^{0^{\operatorname{tr}}} \phi_{t}+h_{t}\left(\left(z-\phi_{t}\right)^{\operatorname{tr}} A_{t}^{-1}\left(z-\phi_{t}\right)\right)^{1 / 2} \\
f^{\theta}(t, z):= & \Pi_{t}^{\perp}\left(\theta_{t}\right)^{\operatorname{tr}} \Pi_{t}^{\perp}(z)-\xi_{t}^{0^{\operatorname{tr}}} \Pi_{t}(z) \\
& +\left(h_{t}^{2}-\xi_{t}^{\theta^{\operatorname{tr}}} A_{t} \xi_{t}^{\theta}\right)^{1 / 2}\left(\Pi_{t}^{\perp}(z)^{\operatorname{tr}} A_{t}^{-1} \Pi_{t}^{\perp}(z)\right)^{1 / 2}
\end{aligned}
$$

It is straightforward to derive the BSDE descriptions for $\pi^{u, \theta}(X)$ and $\rho^{\theta}(X)$ stated in the subsequent proposition. The proof is analogous to that for Theorem 3.8, using (4.10) instead of (3.10), replacing $P$ by $P^{\theta}$ and changing measure from $P^{\theta}$ to $P_{0}$. 
Proposition 4.10. Assume (3.5) and (4.10) hold. For $X \in L^{2}\left(P_{0}\right), \theta \in \Theta$ and $\phi \in \Phi$, let $\left(Y^{\phi, \theta}, Z^{\phi, \theta}\right)$ and $\left(Y^{\theta}, Z^{\theta}\right)$ be the standard solutions to the BSDEs (4.13) and (4.14) respectively. Then $\pi_{t}^{u, \theta}(X)=$ $Y_{t}^{\theta}=E_{t}^{\bar{Q}^{\theta}}[X], t \in[0, T]$, holds with $\bar{Q}^{\theta} \in \mathcal{Q}^{\text {ngd }}\left(P^{\theta}\right)$ given by $d \bar{Q}^{\theta} / d P_{0}=\mathcal{E}\left(\left(-\xi^{0}+\bar{\eta}^{\theta}\right) \cdot W^{0}\right)$ for

$$
\bar{\eta}_{t}^{\theta}=\left(h_{t}^{2}-\xi_{t}^{\theta^{t r}} A_{t} \xi_{t}^{\theta}\right)^{1 / 2}\left(\Pi_{t}^{\perp}\left(Z_{t}^{\theta}\right)^{t r} A_{t}^{-1} \Pi_{t}^{\perp}\left(Z_{t}^{\theta}\right)\right)^{-1 / 2} A_{t}^{-1} \Pi_{t}^{\perp}\left(Z_{t}^{\theta}\right)+\Pi_{t}^{\perp}\left(\theta_{t}\right) .
$$

Moreover $Y_{t}^{\phi, \theta}=\rho_{t}^{\theta}\left(X-\int_{t}^{T} \phi_{s}^{t r} d \widehat{W}_{s}^{0}\right)$ holds, and the strategy $\bar{\phi}^{\theta}$ (in $\left.\Phi\right)$ with

$$
\begin{gathered}
\bar{\phi}_{t}^{\theta}:=\Pi_{t}\left(Z_{t}^{\theta}\right)+\left(\Pi_{t}^{\perp}\left(Z_{t}^{\theta}\right)^{t r} A_{t}^{-1} \Pi_{t}^{\perp}\left(Z_{t}^{\theta}\right)\right)^{1 / 2}\left(h_{t}^{2}-\xi_{t}^{\theta t r} A_{t} \xi_{t}^{\theta}\right)^{-1 / 2} A_{t} \xi_{t}^{\theta} P \otimes d t \text {-a.e. } \\
\text { satisfies } \pi_{t}^{u, \theta}(X)=\rho_{t}^{\theta}\left(X-\int_{t}^{T}\left(\bar{\phi}_{s}^{\theta}\right)^{t r} d \widehat{W}_{s}^{0}\right)=\underset{\phi \in \Phi}{\operatorname{essinf} \rho_{t}^{\theta}\left(X-\int_{t}^{T} \phi_{s}^{t r} d \widehat{W}_{s}^{0}\right) .}
\end{gathered}
$$

By Proposition 4.10, we can write $\pi^{u}(X)$ from (4.12) as

$$
\pi_{t}^{u}(X)=\underset{\theta \in \Theta}{\operatorname{ess} \sup } \underset{\phi \in \Phi}{\operatorname{essinf}} \rho_{t}^{\theta}\left(X-\int_{t}^{T} \phi_{s}^{\text {tr }} d \widehat{W}_{s}^{0}\right), \quad t \in[0, T] .
$$

This permits to describe $\pi^{u}(X)$ and the associated hedging strategy $\bar{\phi}$ in the next theorem by the solution to the classical BSDE

$$
-d Y_{t}=f\left(t, Z_{t}\right) d t-Z_{t}^{\mathrm{tr}} d W_{t}^{0}, t \leq T \quad \text { and } \quad Y_{T}=X
$$

with generator $f(t, \omega, z):=\sup _{\theta \in \Theta} f^{\theta}(t, \omega, z), z \in \mathbb{R}^{n},(t, \omega) \in[0, T] \times \Omega$, with $f^{\theta}$ given by (4.16). The theorem moreover identifies by $\bar{\theta}$ the worst-case model $P^{\bar{\theta}} \in \mathcal{R}$.

Theorem 4.11. Assume (3.5) and (4.10) hold. For $X \in L^{2}\left(P_{0}\right)$, let $(Y, Z)$ be the standard solution to the BSDE (4.18). Then there exists a unique predictable selection $\bar{\theta}:=\bar{\theta}(X)$ of $\Theta$ satisfying $f^{\bar{\theta}}\left(t, Z_{t}\right)=\operatorname{ess}_{\sup } \in \Theta f^{\theta}\left(t, Z_{t}\right), P \otimes d t$-a.e. such that for all $t \in[0, T]$

$$
\pi_{t}^{u}(X)=\rho_{t}^{\bar{\theta}}\left(X-\int_{t}^{T} \bar{\phi}_{s}^{t r} d \widehat{W}_{s}^{0}\right)=\pi_{t}^{u, \bar{\theta}}(X)=Y_{t}
$$

holds with $\bar{\phi}=\left(\bar{\phi}_{t}\right)_{t \in[0, T]}:=\bar{\phi}^{\bar{\theta}}(X) \in \Phi$ given $P \otimes d t$-a.e. by

$$
\bar{\phi}_{t}=\Pi_{t}\left(Z_{t}\right)+\left(\Pi_{t}^{\perp}\left(Z_{t}\right)^{t r} A_{t}^{-1} \Pi_{t}^{\perp}\left(Z_{t}\right)\right)^{1 / 2}\left(h_{t}^{2}-\xi_{t}^{\bar{\theta}^{t r}} A_{t} \xi_{t}^{\bar{\theta}}\right)^{-1 / 2} A_{t} \xi_{t}^{\bar{\theta}} .
$$

The tracking error $R^{\bar{\phi}}(X):=\pi^{u}(X)-\pi_{0}^{u}(X)-\bar{\phi} \cdot \widehat{W}^{0}$ of the strategy $\bar{\phi}$ is a supermartingale under any $Q$ in $\mathcal{P}^{n g d}\left(P^{\bar{\theta}}\right)$, and is a martingale under $\bar{Q}$ in $\mathcal{P}^{n g d}\left(P^{\bar{\theta}}\right)$ given by $d \bar{Q} / d P_{0}=\mathcal{E}\left(\bar{\lambda} \cdot W^{0}\right)$ with

$$
\bar{\lambda}:=h\left((Z-\bar{\phi}) A^{-1}(Z-\bar{\phi})\right)^{-1 / 2} A^{-1}(Z-\bar{\phi})+\bar{\theta} .
$$

Proof. Pointwise existence and uniqueness of $\bar{\theta} \in \Theta$ follow by the continuity and strict concavity of $f^{\theta}$ as a function of $\theta \in \mathbb{R}^{n}$, and the uniform boundedness of $\Theta$. Predictability of $\bar{\theta}$ follows by Rockafellar (1976). The claims (4.19) and (4.20) are corollaries of Proposition 4.10. The remaining claims are similar to those of Theorem 3.8, hence their proof goes likewise, making again use of Lemma 5.1 (instead of Becherer, 2009, Lem.6.1) and (3.5) and (4.10).

The process $\bar{\phi}:=\bar{\phi}^{\bar{\theta}}$ in Theorem 4.11 is the good-deal hedging strategy of $X$ for the worst-case model $P^{\bar{\theta}} \in \mathcal{R}$ which yields that highest good-deal valuation with $\pi^{u}(X)=\pi^{u, \bar{\theta}}(X)$. The tracking error of $\bar{\phi}$ is therefore a supermartingale under any measure in $\mathcal{P}^{\text {ngd }}\left(P^{\bar{\theta}}\right)$ (cf. Proposition 3.3), i.e. $\bar{\phi}$ is "at least mean-self-financing" under any measure in $\mathcal{P}^{\text {ngd }}\left(P^{\bar{\theta}}\right)$. However, it is not clear at this stage whether the supermartingale property of the tracking error of $\bar{\phi}$ holds simultaneously under all measures in $\mathcal{P}^{\text {ngd }}\left(P^{\theta}\right)$ for all models $\mathcal{R}=\left\{P^{\theta}: \theta \in \Theta\right\}$. We will show that this is the case, and that $\bar{\phi}$ and its associated valuation bound $\pi^{u}(X)$ are indeed robust with respect to uncertainty. The idea is first to find an alternative bound $\pi^{u, *}(\cdot)$ and an associated strategy $\phi^{*}$ that satisfy the supermartingale property of the tracking error simultaneously under all measures in $\bigcup_{\theta \in \Theta} \mathcal{P}^{\text {ngd }}\left(P^{\theta}\right)$ and are therefore robust. After this, we show that $\pi^{u, *}(X)$ coincides with the worst-case bound $\pi^{u}$. $(X)$, and that the same holds for the hedging strategies $\bar{\phi}(X)$ and $\phi^{*}(X)$ for any contingent claim $X$. In general the good-deal bound $\pi^{u}(X)$ is dominated by $\pi^{u, *}(X)$, but thanks to a saddle point result (Theorem 4.13) 
one can actually prove that the two bounds are identical. Exchanging the order between ess sup and ess inf in the expression (4.17) for $\pi^{u}(X)$, we define for $X \in L^{2}\left(P_{0}\right)$ and $t \leq T$

$$
\pi_{t}^{u, *}(X):=\underset{\phi \in \Phi}{\operatorname{ess} \inf } \operatorname{ess} \sup _{\theta \in \Theta} \rho_{t}^{\theta}\left(X-\int_{t}^{T} \phi_{s}^{\mathrm{tr}} d \widehat{W}_{s}^{0}\right) .
$$

From this it is clear that in general $\pi_{t}^{u, *}(X) \geq \pi_{t}^{u}(X)$, for all $X \in L^{2}\left(P_{0}\right)$. We will show that in fact the minimax identity holds in the sense that the expressions in (4.17) and (4.21) coincide, and that a saddle point exists, giving equality of $\pi^{u}(X)$ and $\pi^{u, *}(X)$. To this end, we describe $\pi^{u, *}(X)$ and $\phi^{*}$ in terms of the standard solution $(Y, Z)$ for the classical BSDE

$$
-d Y_{t}=f^{*}\left(t, Z_{t}\right) d t-Z_{t}^{\operatorname{tr}} d W_{t}^{0}, \quad t \leq T \quad \text { and } \quad Y_{T}=X
$$

where the generator $f^{*}$ satisfies $P \otimes d t$-a.e. $f^{*}(t, z)=\operatorname{ess} \inf _{\phi \in \Phi} f^{\phi}(t, z)$ for all $z \in \mathbb{R}^{n}$, with $f^{\phi}(\cdot, \cdot, z):=$ $\sup _{\theta \in \Theta} f^{\phi, \theta}(\cdot, \cdot, z)$ for $f^{\phi, \theta}$ from (4.15). Indeed such a generator function $f^{*}$ can be defined at first $P \otimes d t$-a.e. for each $z \in \mathbb{Q}^{n}$, and by Lipschitz continuous extension then $P \otimes d t$-a.e. for all $z \in \mathbb{R}^{n}$. We have

$$
f^{\phi}(t, z)=-\xi_{t}^{0 \operatorname{tr}} \phi_{t}+\sup _{\theta \in \Theta} \theta_{t}^{\operatorname{tr}}\left(z-\phi_{t}\right)+h_{t}\left(\left(z-\phi_{t}\right)^{\operatorname{tr}} A_{t}^{-1}\left(z-\phi_{t}\right)\right)^{1 / 2}
$$

and we can identify the robust good-deal hedging strategy $\phi^{*}$ by

Proposition 4.12. Assume (3.5) and (4.10) hold. For $X \in L^{2}\left(P_{0}\right)$, let $(Y, Z)$ be the standard solution to the BSDE (4.22). Then there exists a unique $\phi^{*} \in \Phi$ satisfying $f^{\phi^{*}}\left(t, Z_{t}\right)=$ ess inf $_{\phi \in \Phi} f^{\phi}\left(t, Z_{t}\right) P \otimes d t$ a.e. such that, for $t \in[0, T]$,

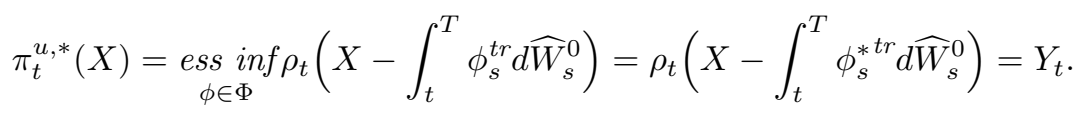

Moreover $R^{\phi^{*}}(X):=\pi^{u, *}(X)-\pi_{0}^{u, *}(X)-\phi^{*} \cdot \widehat{W}^{0}$ is a $Q$-supermartingale for all $Q \in \mathcal{P}^{\text {ngd }}$, and a $Q^{*}$-martingale for $Q^{*} \in \mathcal{P}^{\text {ngd }}$ with $d Q^{*} / d P_{0}=\mathcal{E}\left(\lambda^{*} \cdot W^{0}\right)$, where

$$
\lambda^{*}=h\left(\left(Z-\phi^{*}\right)^{t r} A^{-1}\left(Z-\phi^{*}\right)\right)^{-1 / 2} A^{-1}\left(Z-\phi^{*}\right)+\theta^{*},
$$

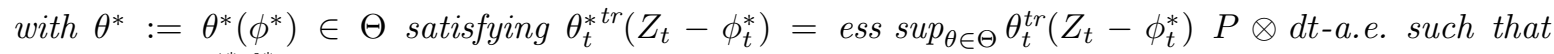
$f^{*}\left(t, Z_{t}\right)=f^{\phi^{*}, \theta^{*}}\left(t, Z_{t}\right), P \otimes d t$-a.e..

Proof. By (Rockafellar, 1976, Thms.2.K and 1.C) one has for any $\phi \in \Phi$ and $z \in \mathbb{R}^{n}$ that there exists $\theta^{*}(\phi)=\theta^{*}(\phi, z) \in \Theta$ such that $\theta_{t}^{*}(\phi)^{\operatorname{tr}}\left(z-\phi_{t}\right)=\sup _{\theta \in \Theta} \theta_{t}^{\operatorname{tr}}\left(z-\phi_{t}\right)$ for all $(t, \omega)$ and hence $f^{\phi}(t, \omega, z)=f^{\phi, \theta^{*}(\phi)}(t, \omega, z)$ for all $(t, \omega)$. Consider the convex continuous function

$$
\mathbb{R}^{n} \ni \phi \mapsto F(\phi):=-\xi^{0^{\operatorname{tr}}} \phi+\sup _{\theta \in \Theta} \theta^{\operatorname{tr}}(z-\phi)+h\left((z-\phi)^{\operatorname{tr}} A^{-1}(z-\phi)\right)^{1 / 2},
$$

for constant $h, \phi, z, \xi^{0}, \sigma$ and $A$ satisfying the notations of Lemma 5.1 and for a compact set $\Theta \subset \mathbb{R}^{n}$ containing the origin. The function $F$ is also coercive on $\operatorname{Im} \sigma^{\operatorname{tr}}$, i.e. $F(\phi) \rightarrow+\infty$ as $|\phi| \rightarrow+\infty$ for $\Pi^{\perp}(\phi)=0$ because $\left|\xi^{0}\right|<h \sqrt{\alpha^{\prime}}$ and $\sup _{\theta \in \Theta} \theta^{\operatorname{tr}}(z-\phi) \geq 0$. Hence existence of $\phi^{*} \in \Phi$ follows from (Ekeland and Temam, 1999, Ch.II, Prop.1.2). Uniqueness of $\phi^{*}$ follows from the fact that $F$ is strictly convex over $\left\{\Pi^{\perp}(\phi)=0\right\}$ if $\Pi^{\perp}(z) \neq 0$ and strictly convex at $\phi=z$ if $\Pi^{\perp}(z)=0$ because (4.10) holds. Finally, predictability of $\phi^{*}$ follows from (Rockafellar, 1976, Thm.2.K) via Part 1 of Proposition 2.2.

From Proposition 4.10, for $\phi \in \Phi$ and $\theta \in \Theta, Y^{\phi, \theta}=\rho^{\theta} .\left(X-\int_{.}^{T} \phi_{s}^{\operatorname{tr}} d \widehat{W}_{s}^{0}\right)$ is the $Y$-component of the solution to the classical BSDE (4.13). As a consequence for every $\phi \in \Phi$ it holds $\operatorname{ess}_{\sup } \operatorname{su}_{\theta \in \Theta} f^{\phi, \theta}\left(t, Z_{t}\right)=$ $f^{\phi, \theta^{*}(\phi)}\left(t, Z_{t}\right)=f^{\phi}\left(t, Z_{t}\right), P \otimes d t$-a.e.. The generators $f^{\phi}$ are standard, so that by the comparison theorem for classical BSDEs, $\left(Y^{\phi}, Z^{\phi}\right)$ with $Y_{t}^{\phi}:=\operatorname{ess}_{\sup } \operatorname{se}_{\theta \in \Theta} Y_{t}^{\phi, \theta}$ is the standard solution to the BSDEs (under $P_{0}$ ) with parameters $\left(f^{\phi}, X\right)$, for $\phi \in \Phi$. The generator $f^{*}$ is also standard because

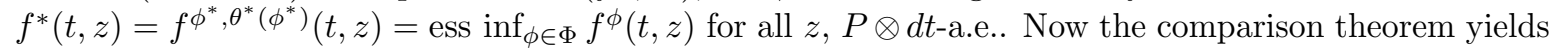
(4.24) from (4.21).

The supermartingale property of $R^{\phi^{*}}(X)$ under any $Q \in \mathcal{P}^{\text {ngd }}$ follows from (4.24) using arguments as in the proof of Proposition 3.3. The martingale property under $Q^{*}$ is proved similarly, noting that the finite variation part under $Q^{*}$ of $R^{\phi^{*}}(X)$ vanishes since $f^{*}\left(t, Z_{t}\right)=f^{\phi^{*}, \theta^{*}}\left(t, Z_{t}\right) P \otimes d t$-a.e.. 
Proposition 4.12 shows that the tracking error of the hedging strategy $\phi^{*}$ with respect to valuation according to $\pi^{u, *}(X)$ has the supermartingale property simultaneously under all measures in $\mathcal{P}^{\text {ngd }}=$ $\bigcup_{\theta \in \Theta} \mathcal{P}^{\text {ngd }}\left(P^{\theta}\right)$. The next theorem shows that a minimax identity holds: the sup-inf representation of $\pi^{u}(\cdot)$ in (4.17) is equal to the inf-sup representation of $\pi^{u, *}(\cdot)$ in (4.21); see also (4.25). Moreover, the good-deal hedging strategy $\bar{\phi}$ with respect to the worst-case model (given by $\bar{\theta}$ ) that gives the highest good-deal valuation bound $\pi_{.}^{u}(\cdot)$, is identical with the robust good-deal hedging strategy $\phi^{*}$ from Proposition 4.12.

Theorem 4.13. Assume (3.5) and (4.10) hold. For $X \in L^{2}\left(P_{0}\right)$, let $(Y, Z)$ be standard solution of the $B S D E$ (4.22). Then $P \otimes d t$-almost everywhere holds

$$
f^{\phi^{*}, \theta^{*}}\left(t, Z_{t}\right)=\underset{\phi \in \Phi}{\operatorname{ess} \inf } \underset{\theta \in \Theta}{\operatorname{ess} \sup } f^{\phi, \theta}\left(t, Z_{t}\right)=\underset{\theta \in \Theta}{\operatorname{ess} \sup } \underset{\phi \in \Phi}{\operatorname{ess} \inf ^{\phi, \theta}}\left(t, Z_{t}\right)=f^{\bar{\phi}, \bar{\theta}}\left(t, Z_{t}\right),
$$

with $(\bar{\phi}, \bar{\theta}),\left(\phi^{*}, \theta^{*}\right)$ from Theorem 4.11 and Proposition 4.12. Moreover $(Y, Z)$ coincides with the standard solution to the BSDE (4.18) and

$$
\pi_{t}^{u}(X)=\pi_{t}^{u, *}(X)=Y_{t}, t \in[0, T] \quad \text { and } \quad \phi_{t}^{*}(X)=\bar{\phi}_{t}(X), P \otimes d t \text {-a.e.. }
$$

Proof. Let $X \in L^{2}\left(P_{0}\right)$. By an application of Lemma 5.2, the generator $f^{\phi, \theta}$ of the BSDE (4.13) for $\theta \in \Theta$ and $\phi \in \Phi$ satisfy the minimax relation (4.25). By Theorem 4.11 and Proposition 4.12 it holds $f\left(t, Z_{t}\right)=f^{\bar{\phi}, \bar{\theta}}\left(t, Z_{t}\right)$ and $f^{*}\left(t, Z_{t}\right)=f^{\phi^{*}, \theta^{*}}\left(t, Z_{t}\right), P \otimes d t$-a.e., for $f, f^{*}$ respectively generators of the BSDEs (4.18), (4.22). Also, $\pi_{t}^{u}(X)=\pi_{t}^{u, *}(X)=Y_{t}, t \in[0, T]$, since by uniqueness of BSDE solutions $(Y, Z)$ also solves the $\operatorname{BSDE}(4.18)$. Hence $(\bar{\phi}, \bar{\theta})$ and $\left(\phi^{*}, \theta^{*}\right)$ are both saddle points of the function $\left(\phi_{t}, \theta_{t}\right) \mapsto f^{\phi, \theta}\left(t, Z_{t}\right)$. Now for any $\theta \in \Theta$ and $z \in \mathbb{R}^{n}$, the function $\phi \mapsto F(\phi, \theta):=$ $\theta^{\operatorname{tr}}(z-\phi)-\xi^{0^{\operatorname{tr}}} \phi+h\left((z-\phi)^{\operatorname{tr}} A^{-1}(z-\phi)\right)^{1 / 2}$ is strictly convex over $\left\{\Pi^{\perp}(\phi)=0\right\}$ if $\Pi^{\perp}(z) \neq 0$, and strictly convex at $\phi=z$ if $\Pi^{\perp}(z)=0$, since $\left|\xi^{\theta}\right|<h \sqrt{\alpha^{\prime}}$. Ekeland and Temam (1999, Ch.VI, Prop.1.5) implies that the $\phi$-components of the saddle points are identical, yielding $\bar{\phi}=\phi^{*}$.

4.5. The impact of model uncertainty on robust good-deal hedging. In the framework of Section 4.4, results have so far been stated for an arbitrary standard correspondence $\Theta$ without further structural assumptions, and ellipsoidal correspondences were only assumed for the no-good-deal restrictions $C^{\theta}, \theta \in \Theta$. Recall (cf. Theorem 3.8 and subsequent remarks) that in the absence of uncertainty the good-deal hedging strategy contains a speculative component in the direction of the market price of risk. This already indicates that under uncertainty one should expect to see relevant differences by a robust approach to hedging. We investigate the effect of uncertainty (solely) about the market prices of risk $\xi^{\theta}$ on robust good-deal hedging, assuming in addition (note that $\xi^{\theta} \in \operatorname{Im} \sigma^{\text {tr }}$ is natural) that for all $(t, \omega) \in[0, T] \times \Omega$, the set $\Theta_{t}(\omega)$ is a subset of $\operatorname{Im} \sigma_{t}^{\mathrm{tr}}(\omega)$, i.e. that

$$
\Theta_{t}(\omega)=\Theta_{t}^{0}(\omega) \cap \operatorname{Im} \sigma_{t}^{\operatorname{tr}}(\omega)
$$

holds for some standard correspondence $\Theta^{0}$ with $0 \in \Theta^{0}$ satisfying the uniform boundedness Assumption 2.3. With (4.27), one clearly has $\Pi^{\perp}(\theta)=0$ for all $\theta \in \Theta$, which implies that $\xi^{\theta}=\xi^{0}+\theta$ for all $\theta \in \Theta$. This leads to the following simplified expressions of the BSDE generators $f^{\phi, \theta}, f^{\theta}$ :

$$
\begin{aligned}
f^{\phi, \theta}(t, z) & =\theta_{t}^{\operatorname{tr}}\left(\Pi_{t}(z)-\phi_{t}\right)-\xi_{t}^{0^{\operatorname{tr}}} \phi_{t}+h_{t}\left(\left(z-\phi_{t}\right)^{\operatorname{tr}} A_{t}^{-1}\left(z-\phi_{t}\right)\right)^{1 / 2} \\
f^{\theta}(t, z) & =-\xi_{t}^{0^{\operatorname{tr}}} \Pi_{t}(z)+\left(h_{t}^{2}-\xi_{t}^{\theta^{\operatorname{tr}}} A_{t} \xi_{t}^{\theta}\right)^{1 / 2}\left(\Pi_{t}^{\perp}(z)^{\operatorname{tr}} A_{t}^{-1} \Pi_{t}^{\perp}(z)\right)^{1 / 2}
\end{aligned}
$$

As a consequence, the process $\bar{\theta}=\bar{\theta}(X)$ does actually not depend on the contingent claim $X \in L^{2}\left(P_{0}\right)$ under consideration, and solves the minimization problem

$$
\xi_{t}^{\bar{\theta}} A_{t} \xi_{t}^{\bar{\theta}}=\min _{\theta \in \Theta} \xi_{t}^{\theta^{\operatorname{tr}}} A_{t} \xi_{t}^{\theta}, \quad t \in[0, T]
$$

In addition in this case, one has $\mathcal{Q}^{\text {ngd }}\left(P^{\bar{\theta}}\right)=\bigcup_{\theta \in \Theta} \mathcal{Q}^{\text {ngd }}\left(P^{\theta}\right)=\mathcal{Q}^{\text {ngd }}$. To obtain even more explicit results one may assume e.g. ellipsoidal uncertainty

$$
\Theta_{t}^{0}(\omega):=\left\{x \in \mathbb{R}^{n} \mid x^{\mathrm{tr}} B_{t}(\omega) x \leq \delta_{t}^{2}(\omega)\right\} \quad \text { for all }(t, \omega) \in[0, T] \times \Omega,
$$

with $\delta$ being a positive bounded and predictable process, and $B$ being a uniformly elliptic and predictable matrix-valued process, satisfying the separability condition (3.5) with respect to $\sigma$. Clearly $f^{\phi}\left(t, Z_{t}\right)$ 
from (4.23) in this case is $P \otimes d t$-a.e. equal to

$$
-\xi_{t}^{0^{\operatorname{tr}}} \phi_{t}+\delta_{t}\left(\left(\Pi_{t}\left(Z_{t}\right)-\phi_{t}\right)^{\operatorname{tr}} B_{t}^{-1}\left(\Pi_{t}\left(Z_{t}\right)-\phi_{t}\right)\right)^{1 / 2}+h_{t}\left(\left(Z_{t}-\phi_{t}\right)^{\operatorname{tr}} A_{t}^{-1}\left(Z_{t}-\phi_{t}\right)\right)^{1 / 2} .
$$

In terms of $\phi^{*}$ and the solution $(Y, Z)$ to the BSDE (4.22), the process $\theta^{*}=\theta^{*}\left(\phi^{*}\right)$ of Proposition 4.12 is given by

$$
\theta_{t}^{*}(X)=\delta_{t}\left(\left(\Pi_{t}\left(Z_{t}\right)-\phi_{t}^{*}\right)^{\operatorname{tr}} B_{t}^{-1}\left(\Pi_{t}\left(Z_{t}\right)-\phi_{t}^{*}\right)\right)^{-1 / 2} B_{t}^{-1}\left(\Pi_{t}\left(Z_{t}\right)-\phi_{t}^{*}\right) .
$$

Remark 4.14. Let us recall Remark 4.7 b). In the present context of Section 4.5 with constraints of ellipsoidal type for good-deals (4.9) and for model uncertainty (4.29), results as explicit as in Section 3.1 can be obtained in particular cases, as elaborated subsequently, but not in general. Indeed, using $\xi^{\theta}=\xi^{0}+\theta$ for $\theta \in \Theta$, to find the minimizer $\bar{\theta}$ (the worst-case) in (4.28) requires to compute the projection of $-\xi_{t}^{0}$ onto the ellipsoid $\Theta_{t}$ with respect to the norm induced by the matrix $A_{t}$. In the radial case $A \equiv I d_{\mathbb{R}^{n}}$ the projection is Euclidian. While there is no closed formula for the projection in general, the solution is described by a parametric formula in terms of a Lagrangian multiplier that solves a 1-dimensional equation, and it can be computed by efficient algorithms (see Kiseliov, 1994), which is relevant if it may be required frequently (as in Monte Carlo simulation, cf. Section 3.2.2).

It is instructive to look at the special case where in addition the matrices $A$ and $B$ are related through $B=A / r$ for some scalar $r>0$; in other words, $B$ basically equals $A$ up to a change of $\delta$ to $\sqrt{r} \delta$. In this case (4.28) is solved by

$$
\bar{\theta}_{t}=-\xi_{t}^{0} I_{\left\{\xi_{t}^{0 \operatorname{tr}} A_{t} \xi_{t}^{0} \leq r \delta_{t}^{2}\right\}}-\frac{\sqrt{r} \delta_{t}}{\left(\xi_{t}^{0 \text { tr }} A_{t} \xi_{t}^{0}\right)^{1 / 2}} \xi_{t}^{0} I_{\left\{\xi_{t}^{0 \operatorname{tr}} A_{t} \xi_{t}^{0}>r \delta_{t}^{2}\right\}}, \quad t \in[0, T],
$$

and replacing $\phi^{*}=\bar{\phi}$ in the formula of $\theta^{*}$ in (4.30) by its expression from (4.20) in terms of $\bar{\theta}$ one obtains $\theta^{*}=\bar{\theta}$. Note that (4.31) implies that $\bar{\theta}^{\operatorname{tr}} A \bar{\theta}$ is equal to $\xi^{{ }^{\operatorname{tr}}} A \xi^{0}$ on $\left\{\xi^{0^{\operatorname{tr}}} A \xi^{0} \leq r \delta^{2}\right\}$ and equal to $r \delta^{2}$ on $\left\{\xi^{0^{\operatorname{tr}}} A \xi^{0}>r \delta^{2}\right\}$. In other words, the worst-case Girsanov kernel $-\bar{\theta}$ is equal to the market price of risk $\xi^{0}$ of the center $P_{0}$ of the confidence set $\mathcal{R}$ of reference measures, being truncated outside a suitable neighborhood $\left\{\xi^{0^{\operatorname{tr}}} A \xi^{0} \leq r \delta^{2}\right\}$.

To obtain an intuition about the impact that model uncertainty may have on robust good-deal hedging, let us look at the behavior of the worst-case Girsanov kernel $\bar{\theta}=\theta^{*}$ obtained in (4.31) and the hedging strategy $\bar{\phi}=\phi^{*}$ in (4.20) for varying scaling constant $r$ : As $r$ becomes large, the worst-case Girsanov kernel $-\bar{\theta}$ becomes close to the market price of risk $\xi^{0}$ and $\phi^{*}=\bar{\phi}$ close to $\Pi(Z)$. This shows that as uncertainty becomes overwhelming, the robust good-deal hedging strategy ceases to comprise a speculative component in the direction of the market price of risk. In such a situation one can show that the hedging strategy is the risk-minimizing strategy under the worst-case no-good-deal measure in the worst-case model $P^{\bar{\theta}}$. More precisely, for an arbitrary shape of the correspondence $\Theta^{0}$, if uncertainty is big enough for the confidence set $\mathcal{R}$ of reference measures to contain some risk neutral pricing measure from $\mathcal{M}^{e}$, then robust good-deal hedging does not comprise a speculative component and the holdings $\phi^{*}$ of a hedging strategy in risky assets coincide with those of the globally risk-minimizing strategy by Föllmer and Sondermann (1986) (cf. Schweizer, 2001, Sect.2) under the reference measure for the worst-case valuation (of the claim). In this sense, the eventually non-speculative nature of the robust good-deal hedging strategy under (large) uncertainty offers new theoretical support for the quadratic hedging objective of risk minimization, which may be criticized for giving equal weighting to upside and downside risk. More broadly, it gives support to a common perception (cf. e.g. Lioui and Poncet, 2000) that speculative objectives should be avoided in hedging, in addition to practical considerations like simplification of marking-to-market (uses risk neutral valuation). To make this precise, let us consider the classical BSDE

$$
-d Y_{t}=\left(-\xi_{t}^{0^{\operatorname{tr}}} \Pi_{t}\left(Z_{t}\right)+h_{t}\left(\Pi_{t}^{\perp}\left(Z_{t}\right)^{\mathrm{tr}} A_{t}^{-1} \Pi_{t}^{\perp}\left(Z_{t}\right)\right)^{1 / 2}\right) d t-Z_{t}^{\mathrm{tr}} d W_{t}^{0},
$$

for $t \in[0, T]$ with $Y_{T}=X$. First we prove the following

Proposition 4.15. Assume (3.5) and (4.10) hold and that $\Theta$ satisfies (4.27). For any $X \in L^{2}\left(P_{0}\right)$, let $\left(Y^{X}, Z^{X}\right)$ denote the standard solution of the BSDE (4.32). Then

$$
\pi^{u}(X)=Y^{X} \text { and } \phi^{*}(X)=\Pi\left(Z^{X}\right) \text { for all } X \in L^{2}\left(P_{0}\right)
$$

holds, if and only if $\mathcal{R} \cap \mathcal{M}^{e}(S) \neq \emptyset$. 
Proof. Let $X \in L^{2}\left(P_{0}\right)$. Recall that for $\Theta$ defined in (4.27), $\bar{\theta}$ from Theorem 4.11 does not vary with $X$ and solves the minimization problem (4.28). Now if (4.34) holds, then there exists $\theta \in \Theta$ such that $P^{\theta} \in \mathcal{R} \cap \mathcal{M}^{e}(S) \neq \emptyset$, i.e. $\widehat{Q}^{\theta}=P^{\theta}$, and therefore $\xi^{\theta}=0$. This implies that $\theta=\bar{\theta}=-\xi^{0}$ and hence $\xi^{0} \in \Theta$. As a consequence, the generator $f=f^{\bar{\theta}}$ of the BSDE (4.18) coincides with that of the BSDE (4.32). By uniqueness of standard BSDE solutions follows $\pi^{u}(X)=Y^{X}$. Now from Theorem 4.11 and Theorem 4.13 one obtains that $\phi^{*}=\bar{\phi}=\Pi\left(Z^{X}\right)$. Conversely, suppose that (4.33) holds. Then the generator $f=f^{\bar{\theta}}$ for the $\operatorname{BSDE}$ (4.18) and the one for (4.32) are equal everywhere by Coquet et al. (2002, Thm.7.1 and Rmk.4.1). This implies (since $\Pi^{\perp}(\theta)=0$ for all $\theta \in \Theta$ ) that $\xi^{\bar{\theta}}=0$, i.e. $\widehat{Q}^{\bar{\theta}}=P^{\bar{\theta}}$, and hence $\mathcal{R} \cap \mathcal{M}^{e}(S) \neq \emptyset$.

Now we can make the previously described relation between risk minimization and good-deal hedging under large uncertainty precise. Using Section 2.1 and $d S / S=\sigma d \widehat{W}^{0}$, any Galtchouk-Kunita-Watanabe (GKW) decomposition (cf. Schweizer, 2001) of a continuous local $Q$-martingale $\left(Q\right.$ in $\mathcal{Q}^{\text {ngd }}$ ) wrt. $\sigma \cdot \widehat{W}^{0}$ gives a GKW decomposition wrt. $S$ and vice versa. Note that $\bar{Q}=\bar{Q}(X)$ depends on $X$ in the

Theorem 4.16. Let the assumptions of Proposition 4.15 and (4.34) hold. For $X$ in $L^{2}\left(P_{0}\right)$, let $(Y, Z)$ be the standard solution of the BSDE (4.32). Then $\pi^{u}(X)=Y$ has the GKW decomposition w.r.t. $\sigma \cdot \widehat{W}^{0}$ (and $S=\operatorname{diag}(S) \sigma \cdot \widehat{W}^{0}$, cf. Sect.2.1)

$$
\pi_{t}^{u}(X)=\pi_{0}^{u}(X)+\phi^{*} \cdot \widehat{W}_{t}^{0}+R_{t}^{\phi^{*}}, \quad t \in[0, T]
$$

with $\phi^{*}=\Pi(Z)$. The tracking error $R^{\phi^{*}}(X)=\Pi^{\perp}(Z) \cdot W^{\bar{Q}}$ is a $\bar{Q}$-martingale orthogonal to $\sigma \cdot \widehat{W}$, for $\bar{Q}(X) \in \mathcal{Q}^{n g d}$ given by $d \bar{Q} / d P_{0}=\mathcal{E}\left(\left(-\xi^{0}+\bar{\eta}\right) \cdot W^{0}\right)$ with

$$
\bar{\eta}_{t}=h_{t}\left(\Pi_{t}^{\perp}\left(Z_{t}\right)^{t r} A_{t}^{-1} \Pi_{t}^{\perp}\left(Z_{t}\right)\right)^{-1 / 2} A_{t}^{-1} \Pi_{t}^{\perp}\left(Z_{t}\right), \quad t \in[0, T] .
$$

Proof. From Proposition 4.15 we have $\phi^{*}=\Pi(Z)$ and $\pi^{u}(X)=Y$. By the definitions of $\bar{\eta}$ and $\bar{Q}$, $Y_{t}=Y_{0}+Z \cdot W_{t}^{\bar{Q}}$ holds for all $t \in[0, T]$. As a consequence, one obtains $\pi_{t}^{u}(X)=\pi_{0}^{u}(X)+\phi^{*}$. $\widehat{W}_{t}^{0}+\Pi^{\perp}(Z.) \cdot W_{t}^{\bar{Q}}, t \in[0, T]$. Thus $(4.35)$ holds with $R^{\phi^{*}}(X)=\Pi^{\perp}(Z) \cdot W^{\bar{Q}}$ being a $\bar{Q}$-martingale orthogonal to $S=S_{0}+\int_{0}^{\cdot} \operatorname{diag}\left(S_{t}\right) \sigma_{t} d W_{t}^{\bar{Q}}$ since $\sigma\left(\Pi^{\perp}(Z)\right)=0$. Furthermore since $\phi_{t}^{*} \perp \Pi_{t}^{\perp}\left(Z_{t}\right)$, and $\phi^{*} \cdot \widehat{W}^{0}=\phi^{*} \cdot W^{\bar{Q}}$, then $R^{\phi *}(X)$ is also orthogonal to $\phi^{*} \cdot \widehat{W}^{0}$ under $\bar{Q}$. Therefore (4.35) is the GKW decomposition of $\bar{\pi}^{u}(X)$ under $\bar{Q}$.

A seminal no-trade result by Dow and Werlang (1992) shows that a utility optimizing agent abstains from taking any position in a risky asset if uncertainty is too large. In comparison, the above theorem shows that a good-deal hedger keeps dynamically trading according to the risk minimizing component $\Pi(Z)$ but ceases to accept any speculative component. In a setting quite different to ours, Boyarchenko et al. (2014) demonstrate by numerical computation in an example that relative benefits of dynamic hedging compared to static hedging could decrease if uncertainty increases. This is intuitive, as (cf. e.g. Cont, 2006) static hedges are less exposed to model risk. Proposition 4.15 similarly addresses how increasing uncertainty affects dynamic hedging, but is different in that it offers theoretical conditions under which dynamic good-deal hedging $\phi^{*}$ ceases to comprise a speculative components in order to compensate for exposures to non-spanned risk.

4.6. Example with closed-form solutions under model uncertainty. The usual filtration is generated by a 2-dimensional $P_{0}$-Brownian motion $W^{0}:=\left(W^{0, S}, W^{0, H}\right)^{\text {tr }}$. We consider a single traded risky asset with price $S$ and a non-traded asset with value $H$ modelled under $P_{0}$ for $t \in[0, T]$ by

$$
d S_{t}=S_{t} \sigma^{S}\left(\xi^{0, S} d t+d W_{t}^{0, S}\right), \quad d H_{t}=H_{t}\left(\gamma d t+\beta\left(\rho d W_{t}^{0, S}+\sqrt{1-\rho^{2}} d W_{t}^{0, H}\right)\right)
$$

with $S_{0}, H_{0}>0$, scalars $\sigma^{S}, \beta>0, \gamma, \xi^{0, S} \in \mathbb{R}$ and correlation coefficient $\rho \in[-1,1]$. We derive robust good-deal bounds and hedging strategies in closed-form, for European call options on the non-traded asset and for no-good-deal restriction and uncertainty modelled (as in Section 4.5) using the radial sets $C^{0}=\left\{x \in \mathbb{R}^{2}:|x| \leq h\right\}$ and $\Theta^{0}=\left\{x \in \mathbb{R}^{2}:|x| \leq \delta\right\}$ for scalars $h, \delta \geq 0$. Here one has $\Theta=\Theta^{0} \cap \operatorname{Im} \sigma=[-\delta, \delta] \times\{0\}$, for $\sigma=\left(\sigma^{S}, 0\right)$, and hence $\bar{\xi}^{\theta}=\left(\xi^{\theta, S}, 0\right)^{\operatorname{tr}}:=\left(\xi^{0, \bar{S}}+\theta^{S}, 0\right)^{\operatorname{tr}} \in \operatorname{Im} \sigma$, for models $P^{\theta}$ with $\theta=\left(\theta^{S}, 0\right)^{\text {tr }}$, where $\theta^{S} \in[-\delta, \delta]$. From $(4.31)$, with $A=B \equiv \operatorname{Id}_{\mathbb{R}^{2}}$ and $r=1$, the worst-case model $P^{\bar{\theta}}$ corresponds to

$$
\bar{\theta}^{S}=-\xi^{0, S} I_{\left\{\left|\xi^{0, S}\right| \leq \delta\right\}}-\delta \frac{\xi^{0, S}}{\left|\xi^{0, S}\right|} I_{\left\{\left|\xi^{0, S}\right|>\delta\right\}} .
$$




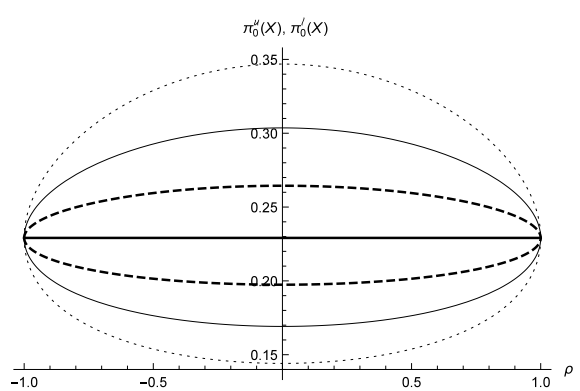

(A) $h_{0}=\xi^{0, S}=0, \quad \delta=0$

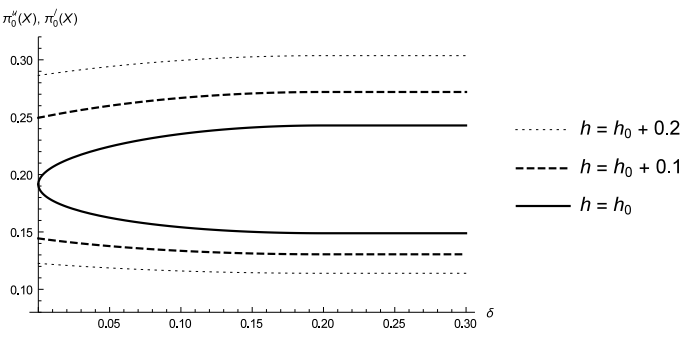

(C) $h_{0}=\xi^{0, S}=0.2, \rho=0.6$

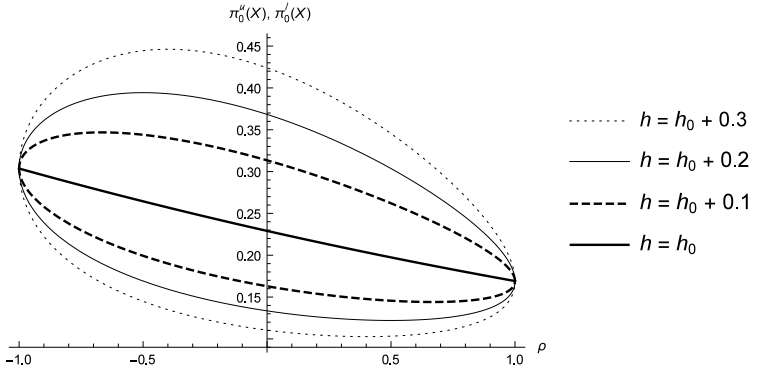

(B) $h_{0}=\xi^{0, S}=0.2, \quad \delta=0$

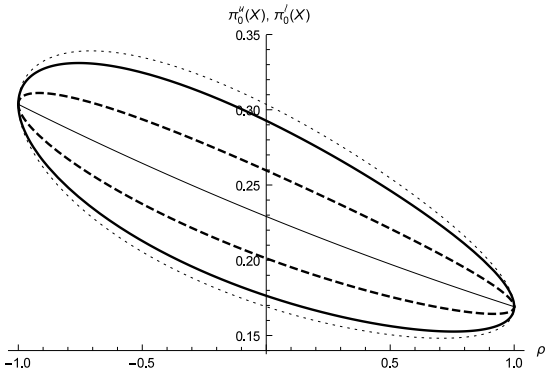

(D) $h=\xi^{0, S}=0.2$

Figure 2. Dependence of $\pi_{0}^{u}(X), \pi_{0}^{l}(X)$ on $\rho, h$ and/or $\delta$

By Theorems 4.11-4.13, the robust good-deal bound and hedging strategy for call option $X:=\left(H_{T}-K\right)^{+}$ are given by $\pi^{u}(X)=Y$ and $\bar{\phi}(X)=\left(Z^{1}+\frac{\left|Z^{2}\right|}{\sqrt{h^{2}-\left|\xi^{\bar{\theta}, S}\right|^{2}}} \xi^{\bar{\theta}, S}, 0\right)^{\operatorname{tr}}$, for $\left(Y, Z:=\left(Z^{1}, Z^{2}\right)^{\operatorname{tr}}\right)$ solving the $\operatorname{BSDE}$ (4.18), equaling the $\operatorname{BSDE}$ (4.14) for $\theta=\bar{\theta}$ :

$$
-d Y_{t}=\left(-\xi^{\bar{\theta}, S} Z_{t}^{1}+\left(h^{2}-\left|\xi^{\bar{\theta}, S}\right|^{2}\right)^{1 / 2}\left|Z_{t}^{2}\right|\right) d t-Z_{t}^{\operatorname{tr}} d W_{t}^{\bar{\theta}} \quad \text { and } \quad Y_{T}=X
$$

with $W_{t}^{\bar{\theta}}:=\left(W_{t}^{0, S}-\bar{\theta}^{S} t, W_{t}^{0, H}\right)^{\operatorname{tr}}, t \in[0, T]$. Writing (4.37) under $\widehat{Q}^{\bar{\theta}}$ and using (4.36), arguments analogous to those in the derivation of (3.14) yield

$$
\begin{aligned}
\pi_{t}^{u}(X) & =N\left(d_{+}\right) H_{t} e^{\tilde{\alpha}_{+}(T-t)}-K N\left(d_{-}\right) \\
& \left.=: \text {B/S-call-price(time: } t, \text { spot: } H_{t} e^{\tilde{\alpha}_{+}(T-t)}, \text { strike: } K, \text { vol: } \beta\right), \\
\pi_{t}^{l}(X) & \left.=\text { B/S-call-price(time: } t, \text { spot: } H_{t} e^{\tilde{\alpha}_{-}(T-t)}, \text { strike: } K, \text { vol: } \beta\right),
\end{aligned}
$$

with $d_{ \pm}:=\left(\ln \left(H_{t} / K\right)+\left(\tilde{\alpha}_{+} \pm \frac{1}{2} \beta^{2}\right)(T-t)\right) /(\beta \sqrt{T-t}), \tilde{\alpha}_{ \pm}:=\gamma+\beta\left(-\rho \xi^{0, S} \pm \tilde{h} \sqrt{1-\rho^{2}}\right)$ and $\tilde{h}:=h I_{\left\{\left|\xi^{0, S}\right| \leq \delta\right\}}+\left(h^{2}-\left|\xi^{0, S}-\delta \xi^{0, S} /\right| \xi^{0, S}||^{2}\right)^{1 / 2} I_{\left\{\left|\xi^{0, S}\right|>\delta\right\}}$. Analogously to the derivation of (3.15), note that $Z=e^{\tilde{\alpha}_{+}(T-t)} N\left(d_{+}\right) H_{t} \beta\left(\rho, \sqrt{1-\rho^{2}}\right)^{\operatorname{tr}}$. Hence the (seller's) robust good-deal hedging strategy is obtained $P \otimes d t$-a.e. as

$$
\bar{\phi}_{t}(X)=e^{\tilde{\alpha}_{+}(T-t)} N\left(d_{+}\right) H_{t} \beta\left(\rho+\frac{\sqrt{1-\rho^{2}} \xi^{0, S}}{\widetilde{h} \xi^{0, S}}\left(\left|\xi^{0, S}\right|-\delta\right) \mathbb{1}_{\left\{\left|\xi^{0, S}\right|>\delta\right\}}, 0\right)^{\operatorname{tr}}, t \in[0, T] .
$$

For $\left|\xi^{0, S}\right|>\delta$, the speculative nature of $\bar{\phi}(X)$ is reflected by the presence of the second summand in the first component of $\bar{\phi}(X)$ above. For $\left|\xi^{0, S}\right| \leq \delta$, this summand vanishes and the function $\delta \mapsto \tilde{\alpha}_{+}$ is constant on $\delta \in\left[\left|\xi^{0, S}\right|, \infty\right]$. In this case robust good-deal hedging is then risk-minimizing and non-speculative as proved in Theorem 4.16. Note that for $\delta=\xi^{0, S}=0$ (i.e. risk-neutral setting under $P_{0}=\widehat{Q}^{0}$ in absence of uncertainty), we recover formulas of Section 3.2.1 for $n=2$ and $d=1$. Figure 2 illustrates the dependence of the bounds $\pi_{0}^{u}(X)$ and $\pi_{0}^{l}(X)$ in the presence of uncertainty, on the correlation coefficient $\rho$, uncertainty size $\delta$ and no-good-deal constraint (optimal growth rate bound) $h$, and for global parameters $\gamma=0.05, \beta=0.5, K=1, H_{0}=1$ and $T=1$. Figures $2 \mathrm{a}, 2 \mathrm{~b}$ are plots of $\pi_{0}^{u}(X)$ and $\pi_{0}^{l}(X)$ as functions of $\rho$ for fixed $\delta=0$ (i.e. absence of uncertainty) and $\xi^{0, S} \in\{0,0.2\}$, showing how the good-deal bounds vary for different values of $h$. Figure $2 d$ contains a similar plot for fixed $h=\xi^{0, S}=0.2$, showing how the bounds vary with $\rho$ for different values of $\delta$. One can observe 
that the maximum of $\pi_{0}^{u}(X)$ and minimum of $\pi_{0}^{l}(X)$ are attained at $\rho=0$ only for $\xi^{0, S}=0$ (cf. Figure 2a). In other words, if the market price of risk $\xi^{0, S}$ is zero (hence $P_{0}=\widehat{Q}^{0}$ ), then the largest good-deal bounds are obtained when the traded and non-traded assets are uncorrelated (i.e. $\rho=0$ ). On the other hand if $\xi^{0, S}>0$ (as e.g. in Figures $2 \mathrm{~b}, 2 \mathrm{~d}$ ), the plots are tilted so that the maximum of $\pi_{0}^{u}(X)$ (resp. minimum of $\left.\pi_{0}^{l}(X)\right)$ is reached at $\rho<0$ (resp. $\rho>0$ ). For $\pi_{0}^{u}(X)$, this is explained by the fact that if the market price of risk $\xi^{0, S}$ is positive, the supremum in (2.3) is maximized by the no-good-deal measure $\bar{Q}=Q^{\bar{\lambda}} \in \mathrm{Q}^{\text {ngd }}\left(P_{0}\right)$ with Girsanov kernel $\bar{\lambda}:=\left(-\xi^{0, S},\left(h^{2}-\left.\left|\xi^{0, S}-\delta \xi^{0, S} /\right| \xi^{0, S}\right|^{2}\right)^{1 / 2}\right)^{\operatorname{tr}}$ under which the upward drift $\tilde{\alpha}_{+}$of the underlying price process $H$ is maximized, clearly at a negative correlation $\rho$. The explanation for $\pi_{0}^{u}(X)$ is similar, with $\tilde{\alpha}_{-}$being minimal at a positive correlation, for $\xi^{0, S}>0$. For $\xi^{0, S}<0$ the tilt of the plots occurs in the other direction. That the good-deal bounds in Figures 2a,2b,2d coincide for perfect correlation $\rho= \pm 1$ is clear, because in this case derivatives $X$ on $H$ are attainable and admit unique no-arbitrage prices, implying $\pi^{u}(X)=\pi^{l}(X)$. Finally, Figure 2c illustrates the evolution with respect to $\delta$ of the good-deal bounds at time $t=0$ for $\rho=0.6, \xi^{0, S}=0.2$ and different values of $h$, with $\left|\xi^{0, S}\right|$ chosen as the smallest value $h_{0}$ of $h$. One observes that for each given $h$, the good-deal bound curves become flat for $\delta \geq\left|\xi^{0, S}\right|$ (as predicted by Proposition 4.15), and match (i.e. $\left.\pi_{0}^{u}(X)=\pi_{0}^{l}(X)\right)$ for $\delta=\left|\xi^{0, S}\right|-h_{0}=0$ (as might be expected in the absence of uncertainty for a degenerate expected growth rate bound $\left.h=\left|\xi^{0, S}\right|\right)$.

\section{APPENDIX}

This section includes lemmas and derivations omitted from the paper's main body.

Lemma 5.1. For $d<n$, let $\sigma \in \mathbb{R}^{d \times n}$ be of full-rank, $A \in \mathbb{R}^{n \times n}$ be symmetric and positive definite, and $h>0, Z \in \mathbb{R}^{n}, \xi \in \operatorname{Im} \sigma^{\text {tr }}$. Let $\alpha^{\prime}>0$ be a constant of ellipticity of $A^{-1}$ and assume that $|\xi|<h \sqrt{\alpha^{\prime}}$ and $A^{-1}(\operatorname{Ker} \sigma)=\operatorname{Ker} \sigma$. Then $\bar{\phi}:=\Pi(Z)+\left(\Pi^{\perp}(Z)^{t r} A^{-1} \Pi^{\perp}(Z)\right)^{1 / 2}\left(h^{2}-\xi^{t r} A \xi\right)^{-1 / 2} A \xi$ is the unique minimizer of the function $\phi \mapsto F(\phi):=-\xi^{*} \phi+h\left((Z-\phi)^{t r} A^{-1}(Z-\phi)\right)^{1 / 2}$ on $\operatorname{Im} \sigma^{t r}$.

Proof. The proof is an application of the classical Kuhn-Tucker Theorem (cf. Rockafellar, 1970, Sect.28). For details see (Kentia, 2015, Lemma 3.35) and proof.

Lemma 5.2. Let $d<n, h>0$ be constant, $Z \in \mathbb{R}^{n}, A \in \mathbb{R}^{n \times n}$ a symmetric positive definite matrix, $\sigma \in \mathbb{R}^{d \times n}$ a full (d)-rank matrix, and $\xi^{0} \in \Phi:=\operatorname{Im} \sigma^{t r}$. Let $\Theta \subset \mathbb{R}^{n}$ be a convex-compact set, and $F: \mathbb{R}^{n} \times \mathbb{R}^{n} \ni(\phi, \theta) \mapsto \theta^{\operatorname{tr}}(Z-\phi)-\xi^{0^{t r}} \phi+h\left((Z-\phi)^{t r} A^{-1}(Z-\phi)\right)^{1 / 2}$. Then the minmax identity $\inf _{\phi \in \Phi} \sup _{\theta \in \Theta} F(\phi, \theta)=\sup _{\theta \in \Theta} \inf _{\phi \in \Phi} F(\phi, \theta)$. holds.

Proof. For all $\phi \in \mathbb{R}^{n}$, the function $\theta \mapsto F(\phi, \theta)$ is concave, continuous. For all $\theta \in \mathbb{R}^{n}$ the function $\phi \mapsto F(\phi, \theta)$ is convex and continuous. As $\Theta \subset \mathbb{R}^{n}$ is convex and compact, and $\Phi=\operatorname{Im} \sigma^{\text {tr }}$ is convex and closed, a minimax theorem (Ekeland and Temam, 1999, Ch.VI, Prop.2.3) applies and the minmax identity holds.

Proof of Lemma 2.1. Part a) is classical (see Delbaen 2006 and cf. previously given other references). As for Part b), m-stability and convexity of $\mathcal{M}^{e}$ follow from (Delbaen, 2006, Prop.5). Convexity of $\mathcal{Q}^{\text {ngd }}$ follows from that of $\mathcal{M}^{e}$ and the values of $C$. To show m-stability of $\mathcal{Q}^{\text {ngd }}$, let $Z^{i}=$ $\mathcal{E}\left(\lambda^{i} \cdot W\right) \in \mathcal{Q}^{\text {ngd }}, i=1,2, \tau \leq T$ be a stopping time and $Z=I_{[0, \tau]} Z^{1}+I_{] \tau, T]} Z_{\tau}^{1} Z_{.}^{2} / Z_{\tau}^{2}$. Since $\mathcal{M}^{e}$ is m-stable, then $Z \in \mathcal{M}^{e}$ and one has $Z=\mathcal{E}(\lambda \cdot W)$ for some predictable process $\lambda$. It remains to show that $\lambda$ is bounded and that $\lambda \in C$. From the expression of $Z$, writing the densities $Z, Z^{1}, Z^{2}$ as ordinary exponentials by distinguishing $t \leq \tau$ and $t \geq \tau$, and taking the logarithm yields $\left(\lambda-I_{[0, \tau]} \lambda^{1}-I_{] \tau, T]} \lambda^{2}\right) \cdot W=\frac{1}{2} \int_{0}^{\cdot}\left(\left|\lambda_{s}\right|^{2}-I_{[0, \tau]}(s)\left|\lambda_{s}^{1}\right|^{2}-I_{] \tau, T]}(s)\left|\lambda_{s}^{2}\right|^{2}\right) d s$. Since $\mathbb{F}$ is the augmented Brownian filtration, then $[0, \tau]$ and $] \tau, T]$ are predictable and so is $\lambda-I_{[0, \tau]} \lambda^{1}-I_{\tau, T]} \lambda^{2}$. Hence $\left(\lambda-I_{[0, \tau]} \lambda^{1}-I_{] \tau, T]} \lambda^{2}\right) \cdot W$ is a continuous local martingale of finite variation and is thus equal to zero. As a consequence $\lambda=I_{[0, \tau]} \lambda^{1}+I_{] \tau, T]} \lambda^{2}$ is bounded since $\lambda^{1}, \lambda^{2}$ are, and satisfies $\lambda \in C$ since $C$ is convex-valued.

Proof of Theorem 2.7. As shown in (Kentia, 2015, Thm.3.7), $\pi^{u}(X)$ admits under $\widehat{Q}$ the Doob-Meyer decomposition $\pi^{u}(X)=\pi_{0}^{u}(X)+Z \cdot \widehat{W}-A=\pi_{0}^{u}(X)+Z \cdot W+\int_{0}^{\cdot} \xi_{t}^{\operatorname{tr}} Z_{t} d t-A$, where $Z \in \mathcal{H}^{2}(\widehat{Q})$ and $A$ is a non-decreasing predictable process with $A_{0}=0$. Alternatively one rewrites $-d \pi_{t}^{u}(X)=$ $g_{t}\left(Z_{t}\right) d t-Z_{t}^{\mathrm{tr}} d W_{t}+d K_{t}$, with $K:=A-\int_{0}^{\cdot} \xi_{t}^{\operatorname{tr}} Z_{t} d t-\int_{0}^{\cdot} \operatorname{ess}_{\sup _{\lambda \in \Lambda}} \lambda_{t}^{\operatorname{tr}} Z_{t} d t$ being finite-valued and predictable. For $\left(\pi^{u}(X), Z, K\right)$ to be a supersolution to the BSDE with parameters $(g, X)$ it suffices 
to show that $K$ is non-decreasing. For any $\lambda=-\xi+\eta \in \Lambda$, one can construct the sequence of $\lambda^{n}=-\xi+\eta^{n} \in \Lambda$ Girsanov kernels of measures $Q^{n} \in \mathcal{Q}^{\text {ngd }}$ with $\eta^{n}=\eta I_{\{|\eta| \leq n\}}$ such that $\lambda^{n} \rightarrow \lambda P \otimes d t$-a.e. as $n \rightarrow \infty$. For each $Q^{n}$ it holds $\pi_{.}^{u}(X)=\pi_{0}^{u}(X)+Z \cdot W^{Q^{n}}+\int_{0}^{-} Z_{t}^{\operatorname{tr}} \eta_{t}^{n} d t-A$. Since $\pi^{u}(X)$ is a bounded $Q^{n}$-supermartingale, then $d A_{t}-\xi_{t}^{\operatorname{tr}} Z_{t} d t \geq Z_{t}^{\operatorname{tr}} \lambda_{t}^{n} d t$, for all $n \in \mathbb{N}$. Taking the limit as $n \rightarrow \infty$ and using dominated convergence one obtains $d A_{t}-\xi_{t}^{\operatorname{tr}} Z_{t} d t \geq Z_{t}^{\operatorname{tr}} \lambda_{t} d t$. Now taking the essential supremum over all $\lambda \in \Lambda$ yields $d K_{t} \geq 0$.

To show that the supersolution $\left(\pi^{u}(X), Z, K\right)$ is minimal, it suffices by the dynamic principle (cf. Kentia, 2015, Lem.3.6) to show that the $Y$-component of any other supersolution is a càdlàg $Q$-supermartingale for any $Q \in \mathcal{Q}^{\text {ngd }}$. Let $(\bar{Y}, \bar{Z}, \bar{K})$ be a supersolution of the BSDE for parameters $(g, X)$, with $\bar{Y} \in \mathcal{S}^{\infty}$. By change of measure, under a $Q \in \mathcal{Q}^{\text {ngd }}$ with Girsanov kernel $\lambda^{Q} \in \Lambda$ we have

$$
\begin{gathered}
-d \bar{Y}_{t}=\left(\underset{\lambda \in \Lambda}{\operatorname{ess} \sup } \lambda_{t}^{\operatorname{tr}} \bar{Z}_{t}-\bar{Z}_{t}^{\operatorname{tr}} \lambda_{t}^{Q}\right) d t-\bar{Z}_{t}^{\operatorname{tr}} d W_{t}^{Q}+d \bar{K}_{t}, \quad t \in[0, T], \\
\text { and get } \quad d \bar{K}_{t}+\left(\underset{\lambda \in \Lambda}{\operatorname{ess} \sup _{t}} \lambda_{t}^{\operatorname{tr}} \bar{Z}_{t}-\bar{Z}_{t}^{\operatorname{tr}} \lambda_{t}^{Q}\right) d t \geq 0, \quad t \in[0, T],
\end{gathered}
$$

by using that $\bar{K}$ is non-decreasing. By (5.1-5.2) and $\bar{Y} \in \mathcal{S}^{\infty}$, the local martingale $\bar{Z} \cdot W^{Q}$ is bounded from below, and thus is a supermartingale. As $\bar{Y} \in \mathcal{S}^{\infty}$, the integral of (5.2) on $[0, T]$ is in $L^{1}(Q)$ and so $\bar{Y}$ is a $Q$-supermartingale.

Derivation of (3.18),(3.20). The stochastic exponential $\mathcal{E}\left((\varepsilon / \sqrt{\nu}) \cdot W^{\nu}\right)$ is a uniformly integrable martingale which defines a measure $\bar{Q} \in \overline{\mathcal{Q}^{\text {ngd }}} \supseteq \mathcal{Q}^{\text {ngd }}$ (see $(2.4)$ for definition of $\overline{\mathcal{Q}^{\text {ngd }}} \subset \mathcal{M}^{e}$ ) with Girsanov kernel $\bar{\lambda}:=\varepsilon / \sqrt{\nu}$, i.e. $d \bar{Q} / d P=\mathcal{E}\left((\varepsilon / \sqrt{\nu}) \cdot W^{\nu}\right)$. Indeed, applying (Cheridito et al., 2005, Thm.2.4 and Sect.6) one gets that $\mathcal{E}\left((\varepsilon / \sqrt{\nu}) \cdot W^{\nu}\right)$ and $S=S_{0} \mathcal{E}\left(\sqrt{\nu} \cdot W^{S}\right)$ are uniformly integrable $P$ - respectively $Q$-martingales. The variance process $\nu$ under $\bar{Q}$ is again a CIR process with parameters $(\bar{a}, b, \beta, \rho)$ where $\bar{a}:=a+\beta \varepsilon \sqrt{1-\rho^{2}}>a$ and the Feller condition $\beta^{2} \leq 2 \bar{a}$ still holds. For a put option $X=\left(K-S_{T}\right)^{+} \in L^{\infty}, \bar{Y}_{t}:=E_{t}^{\bar{Q}}[X]$ are given by the Heston formula (cf. Heston, 1993), applied under $\bar{Q}$ (instead of $P$ ). Since the Heston price is non-decreasing in the mean reversion level of the variance process (Ould-Aly, 2011, Prop.5.3.1) one expects that $\pi_{t}^{u}(X)=\bar{Y}_{t}=E_{t}^{\bar{Q}}[X]$. Let us make this precise. For $Q \in \overline{\mathcal{Q}^{\text {ngd }}}$ with Girsanov kernel $\lambda$ satisfying $|\lambda| \leq \varepsilon / \sqrt{\nu}$, one has $Y_{T}^{Q}=\bar{Y}_{T}=X$ with $Y_{t}^{Q}=E_{t}^{Q}[X]$. Using Feynman-Kac, $\bar{Y}_{t}=u\left(t, S_{t}, \nu_{t}\right)$ for a function $u \in \mathcal{C}^{1,2,2}\left([0, T] \times \mathbb{R}^{+} \times \mathbb{R}^{+}\right)$with $\frac{\partial u}{\partial \nu} \geq 0$ (see Ould-Aly, 2011, Thm.5.3.1, Cor.5.3.1). By Itô's formula and change of measure follows

$$
\begin{aligned}
d \bar{Y}_{t}= & \beta \sqrt{1-\rho^{2}} \sqrt{\nu_{t}}\left(\lambda_{t}-\frac{\varepsilon}{\sqrt{\nu_{t}}}\right) \frac{\partial u}{\partial \nu}\left(t, S_{t}, \nu_{t}\right) d t+\beta \sqrt{1-\rho^{2}} \sqrt{\nu_{t}} \frac{\partial u}{\partial \nu}\left(t, S_{t}, \nu_{t}\right) d W_{t}^{Q, \nu} \\
& +\left(S_{t} \sqrt{\nu_{t}} \frac{\partial u}{\partial S}\left(t, S_{t}, \nu_{t}\right)+\beta \rho \sqrt{\nu_{t}} \frac{\partial u}{\partial \nu}\left(t, S_{t}, \nu_{t}\right)\right) d W_{t}^{S}, \quad t \in[0, T]
\end{aligned}
$$

Since $X$ is bounded, then $\bar{Y}$ is in $\mathcal{S}^{\infty}(Q)$ and a $Q$-supermartingale by (5.3). Hence $Y_{t}^{Q} \leq \bar{Y}_{t}$ for all $Q \in \overline{\mathcal{Q}^{\text {ngd }}}$, which by Part 1. of Kentia (2015, Thm.3.7) implies the claim and thus we obtain the Heston type formula (3.18).

Since $\bar{Q} \in \overline{\mathcal{Q}^{\text {ngd }}}$ and $\pi_{0}^{u}(X)=E^{\bar{Q}}[X]$ with $X \in L^{\infty}$, Corollary 2.8 implies that the good-deal bound is the $Y$-component of the minimal solution $(\bar{Y}, \bar{Z}) \in \mathcal{S}^{\infty} \times \mathcal{H}^{2}$ (note $P=\widehat{Q}$ ) of the BSDE (3.19) with generator $g_{t}(z)=\bar{\lambda}_{t} z^{2}=\varepsilon z^{2} / \sqrt{\nu_{t}}$, for $z=\left(z^{1}, z^{2}\right)$, and terminal condition $X$. Now consider the strategy

$$
\bar{\phi}_{t}=\bar{Z}_{t}^{1}=S_{t} \sqrt{\nu_{t}} \frac{\partial u}{\partial S}\left(t, S_{t}, \nu_{t}\right)+\beta \rho \sqrt{\nu_{t}} \frac{\partial u}{\partial \nu}\left(t, S_{t}, \nu_{t}\right)=S_{t} \sqrt{\nu_{t}} \Delta_{t}+\frac{\beta \rho}{2} \mathcal{V}_{t}
$$

Clearly $\bar{\phi}$ is in the set $\Phi=\mathcal{H}^{2}(\mathbb{R})$ of permitted trading strategies since $\bar{Z} \in \mathcal{H}^{2}\left(\mathbb{R}^{2}\right)$. Recall that $\mathcal{P}^{\text {ngd }}$ consists of $d Q / d P=\mathcal{E}\left(\left(\lambda^{S}, \lambda^{\nu}\right) \cdot W\right)$ such that $\left|\left(\lambda^{S}, \lambda^{\nu}\right)\right| \leq \varepsilon / \sqrt{\nu}$ with $\left(\lambda^{S}, \lambda^{\nu}\right)$ being bounded. For $Q \in \mathcal{P}^{\text {ngd }}$, any wealth process $\phi \cdot W^{S}, \phi \in \Phi$, is thus in $\mathcal{S}^{1}(Q)$. As $\mathcal{Q}^{\text {ngd }} \subseteq \mathcal{P}^{\text {ngd }}$ holds, clearly $\pi_{t}^{u}(X) \leq \rho_{t}\left(X-\int_{t}^{T} \phi_{s} d W_{s}^{S}\right)$ for any strategy $\phi \in \Phi$. To prove that $\bar{\phi}$ is a good-deal hedging strategy, we show the reverse inequality $\pi_{t}^{u}(X) \geq E_{t}^{Q}\left[X-\int_{t}^{T} \bar{\phi}_{s} d W_{s}^{S}\right]$ for all $Q \in \mathcal{P}^{\text {ngd }}$. Let $Q \in \mathcal{P}^{\text {ngd }}$ with 
Girsanov kernel $\left(\lambda^{S}, \lambda^{\nu}\right)$. Like in (5.3), we obtain for any stopping time $\tau$ that

$$
\begin{aligned}
\bar{Y}_{\tau \wedge T}-\int_{\tau \wedge t}^{\tau \wedge T} \bar{\phi}_{s} d W_{s}^{S}=\bar{Y}_{\tau \wedge t} & +\int_{\tau \wedge t}^{\tau \wedge T} \beta \sqrt{1-\rho^{2}} \sqrt{\nu_{s}}\left(\lambda_{s}^{\nu}-\frac{\varepsilon}{\sqrt{\nu_{s}}}\right) \frac{\partial u}{\partial \nu}\left(s, S_{s}, \nu_{s}\right) d s \\
& +L_{\tau \wedge T}-L_{\tau \wedge t},
\end{aligned}
$$

for the local $Q$-martingale $L:=\int_{0}^{\cdot} \beta \sqrt{1-\rho^{2}} \sqrt{\nu_{s}} \frac{\partial u}{\partial \nu}\left(s, S_{s}, \nu_{s}\right) d W_{s}^{Q, \nu}$. By $\frac{\partial u}{\partial \nu} \geq 0$ and $\left|\lambda^{\nu}\right| \leq \varepsilon / \sqrt{\nu}$ follows that $\bar{Y}_{\tau \wedge T}-\int_{\tau \wedge t}^{\tau \wedge T} \bar{\phi}_{s} d W_{s}^{S}$ is less than $\bar{Y}_{\tau \wedge t}+L_{\tau \wedge T}-L_{\tau \wedge t}$. Localizing $L$ along a sequence of stopping times $\tau_{n} \uparrow \infty$ and taking conditional $Q$-expectations yields $E_{t}^{Q}\left[\bar{Y}_{\tau_{n} \wedge T}-\int_{\tau_{n} \wedge t}^{\tau_{n} \wedge T} \bar{\phi}_{s} d W_{s}^{S}\right] \leq \bar{Y}_{\tau_{n} \wedge t}$. Using $X \in L^{\infty}$ and $\bar{\phi} \cdot W^{S} \in S^{1}(Q)$, the claim then follows by dominated convergence. Hence (3.20) holds for $\mathcal{V}_{t}:=\frac{\partial u}{\partial \sigma}\left(t, S_{t}, \nu_{t}\right)=2 \sigma_{t} \frac{\partial u}{\partial \nu}\left(t, S_{t}, \nu_{t}\right)$ and volatility $\sigma_{t}=\sqrt{\nu_{t}}$.

\section{REFERENCES}

C. D. Aliprantis and K. C. Border. Infinite Dimensional Analysis: A Hitchhiker's Guide. Springer, Berlin, 3rd edition, 2006.

P. Artzner, F. Delbaen, J.-M. Eber, D. Heath, and H. Ku. Coherent multiperiod risk adjusted values and Bellman's principle. Ann. Oper. Res., 152(1):5-22, 2007.

P. Barrieu and N. El Karoui. Pricing, hedging and optimally designing derivatives via minimization of risk measures. In R. Carmona, editor, Indifference Pricing: Theory and Applications, pages 77-146. Princeton University Press, 2009.

E. Bayraktar and V. Young. Pricing options in incomplete equity markets via the instantaneous Sharpe ratio. Ann. Finance, 4(4):399-429, 2008.

D. Becherer. From bounds on optimal growth towards a theory of good-deal hedging. Radon Ser. Comput. Appl. Math., 8:27-52, 2009.

D. Becherer and P. Turkedjiev. Multilevel approximation of backward stochastic differential equations. Preprint, arXiv:1412.3140, 2014.

S. Biagini and M. Ç. Pınar. The robust Merton problem of an ambiguity averse investor. Math. Financ. Econ., pages 1-24, 2016.

J. Bion-Nadal and G. Di Nunno. Dynamic no-good-deal pricing measures and extension theorems for linear operators on $L^{\infty}$. Financ. Stoch., 17(3):587-613, 2013.

T. Björk and I. Slinko. Towards a general theory of good-deal bounds. Rev. Finance, 10:221-260, 2006.

O. Bondarenko and I. Longarela. A general framework for the derivation of asset price bounds: an application to stochastic volatility option models. Rev. Deriv. Res., 12(2):81-107, 2009.

N. Boyarchenko, M. Cerrato, J. Crosby, and S. D. Hodges. No good deals - no bad models. SSRNid2467066, NY Federal Reserve Staff Report No. 589, 2014. http://dx.doi.org/10.2139/ssrn. 2192559.

L. Carassus and E. Temam. Pricing and hedging basis risk under no good deal assumption. Ann. Finance, 10:127-170, 2014.

A. Cerný. Generalized Sharpe ratios and asset pricing in incomplete markets. European Finance Rev., 7:191-233, 2003.

A. Cerný and S. D. Hodges. The theory of good-deal pricing in financial markets. In H. Geman, M. D.P., S. Plinska, and T. Vorst, editors, Mathematical Finance - Bachelier Congress 2000, pages 175-202. Springer, Berlin, 2002.

Z. Chen and L. G. Epstein. Ambiguity, risk and asset returns in continuous time. Econometrica, 70(4): 1403-1443, 2002.

P. Cheridito and M. Kupper. Recursiveness of indifference prices and translation-invariant preferences. Math. Financ. Econ., 2(3):173-188, 2009.

P. Cheridito, D. Filipović, and M. Yor. Equivalent and absolutely continuous measure changes for jump-diffusion processes. Ann. Appl. Probab., 15(3):1713-1732, 2005. ISSN 1050-5164.

J. Cochrane and J. S. Requejo. Beyond arbitrage: Good deal asset price bounds in incomplete markets. J. Polit. Econ., 108:79-119, 2000.

R. Cont. Model uncertainty and its impact on the pricing of derivative instruments. Math. Financ., 16 (3):519-547, 2006.

F. Coquet, Y. Hu, J. Mémin, and S. Peng. Filtration-consistent nonlinear expectations and related g-expectations. Probab. Theory Related Fields, 123(1):1-27, 2002. 
F. Delbaen. The structure of m-stable sets and in particular of the set of risk neutral measures. In Séminaire de Probabilités XXXIX, Lecture Notes in Mathematics 1874, pages 215-258. Springer, Berlin, 2006.

F. Delbaen and W. Schachermayer. A general version of the fundamental theorem of asset pricing. Math. Ann., 300:463-520, 1994.

Ł. Delong. No-good-deal, local mean-variance and ambiguity risk pricing and hedging for an insurance payment process. Astin Bull., 42(01):203-232, 2012.

C. Donnelly. Good-deal bounds in a regime-switching diffusion market. Appl. Math. Finance, 18(6): 491-515, 2011.

J. Dow and S. Werlang. Uncertainty aversion, risk aversion, and the optimal choice of portfolio. Econometrica, pages 197-204, 1992.

S. Drapeau and M. Kupper. Risk preferences and their robust representation. Math. Oper. Res., 38(1): 28-62, 2013.

S. Drapeau, G. Heyne, and M. Kupper. Minimal supersolutions of convex BSDEs. Ann. Probab., 41(6): 3973-4001, 2013.

I. Ekeland and R. Temam. Convex Analysis and Variational Problems. SIAM, 1999.

N. El Karoui and M. Quenez. Dynamic programming and pricing of contingent claims in an incomplete market. SIAM J. Control Optim., 33(1):29-66, 1995.

N. El Karoui, S. Peng, and M. C. Quenez. Backward stochastic differential equations in finance. Math. Finance, 1:1-71, 1997.

L. G. Epstein and M. Schneider. Recursive multiple-priors. J. Econ. Theo., 113(1):1-31, 2003.

H. Föllmer and D. Sondermann. Hedging of non-redundant contingent claims. In W. Hildenbrand and A. Mas-Colell, editors, Contributions to Math.l Economics, pages 205-223. North-Holland, 1986.

L. Garlappi, R. Uppal, and T. Wang. Portfolio selection with parameter and model uncertainty: A multi-prior approach. Review of Financial Studies, 20(1):41-81, 2007.

I. Gilboa and D. Schmeidler. Maxmin expected utility with non-unique prior. J. Math. Econ., 18(2): 141-153, 1989.

E. Gobet and P. Turkedjiev. Linear regression MDP scheme for discrete backward stochastic differential equations under general conditions. Math Comp, 85(299):1359-1391, 2016.

L. P. Hansen and T. J. Sargent. Robust control and model uncertainty. Am. Econ. Rev., 91:60-66, 2001.

D. Heath, E. Platen, and M. Schweizer. A comparison of two quadratic approaches to hedging in incomplete markets. Math. Financ., 11(4):385-413, 2001.

S. L. Heston. A closed-form solution for options with stochastic volatility, with application to bond and currency options. Rev. Financ. Stud., 6:327-343, 1993.

S. Jaschke and U. Küchler. Coherent risk measures and good-deal bounds. Finance Stoch., 5(2):181-200, 2001.

I. Karatzas and S. E. Shreve. Brownian Motion and Stochastic Calculus. Springer, Berlin, 2006.

K. Kentia. Robust aspects of hedging and valuation in incomplete markets and related backward SDE theory. PhD thesis, Humboldt-Universität zu Berlin, 2015. urn:nbn: de:kobv:11-100237580.

Y. N. Kiseliov. Algorithms of projection of a point onto an ellipsoid. Lithuanian Math. J, 34(2):141-159, 1994.

S. Klöppel and M. Schweizer. Dynamic indifference valuation via convex risk measures. Math. Financ., 17(4):599-627, 2007.

S. Klöppel and M. Schweizer. Dynamic utility-based good-deal bounds. Statistics and Decisions, 25: 311-332, 2007.

J. Leitner. Pricing and hedging with globally and instantaneously vanishing risk. Statistics ES Decisions, 25(4):311-332, 2007.

A. Lioui and P. Poncet. The minimum variance hedge ratio under stochastic interest rates. Management Sci., 46(5):658-668, 2000.

P. C. Mahalanobis. On the generalized distance in statistics. Proceedings National Institute of Sciences (Calcutta), 2:49-55, 1936.

N. Marroquín-Martínez and M. Moreno. Optimizing bounds on security prices in incomplete markets. Does stochastic volatility specification matter? Europ. J. Oper. Res., 225(3):429-442, 2013. 
S. Ould-Aly. Modélisation de la Courbe de Variance et Modèles à Volatilité Stochastique. PhD thesis, Université Paris-Est, June 2011. http://tel.archives-ouvertes.fr/tel-00604530/en.

E. Pardoux and S. Peng. Adapted solution of a backward stochastic differential equation. Syst. Control Lett., 14:55-61, 1990.

R. Poulsen, K. R. Schenk-Hopp, and C.-O. Ewald. Risk minimization in stochastic volatility models: model risk and empirical performance. Quant Financ, 9(6):693-704, 2009.

M. C. Quenez. Optimal portfolio in a multiple-priors model. In Seminar on Stochastic Analysis, Random Fields and Applications IV, Progress in Probability, volume 58, pages 291-321. Birkhäuser, Basel, 2004.

R. T. Rockafellar. Convex Analysis. Princeton University Press, Princeton, 1970.

R. T. Rockafellar. Integral functionals, normal integrands and measurable selections. In L. Waelbroeck, editor, Nonlinear Operators and Calculus of Variations, Lecture Notes in Mathematics 543, pages 157-207. Springer, Berlin, 1976.

A. Schied. Robust optimal control for a consumption-investment problem. Math. Method. Oper. Res., $67(1): 1-20,2008$.

M. Schweizer. A guided tour through quadratic hedging approaches. In E. Jouini, J. Cvitanić, and M. Musiela, editors, Option Pricing, Interest Rates and Risk Management, pages 538-574. Cambridge University Press, Cambridge, 2001.

F. T. Seifried. Optimal investment for worst-case crash scenarios: A martingale approach. Math. Oper. Res., 35(3):559-579, 2010.

(D. Becherer) Institut für Mathematik, Humboldt-Universität Zu Berlin, D-10099 Berlin, Germany E-mail address: becherer@mathematik.hu-berlin.de

(K. Kentia) Previous: Institut für Mathematik, Humboldt-Universität Zu Berlin, D-10099 Berlin, Germany Current: Institut für Mathematik, Goethe-Universität Frankfurt, D-60054 Frankfurt a.M., Germany E-mail address: kentia@math.uni-frankfurt.de 